\title{
L'INNOVAZIONE PER LO SVILUPPO LOCALE L'UNIVERSITÀ PER IL TERRITORIO
}

\author{
ATTI DEL CONVEGNO DI STUDI
}

EMPOLI, 12 MARZO 2004

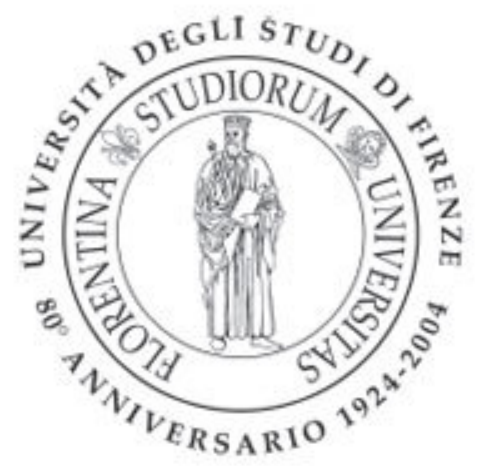

a cura di

ALFIERO CIAMPOLINI

Firenze University Press 
UniversitÀ DEGLI STUdi DI FirenZE

$80^{\circ}$ ANNIVERSARIO 1924-2004

$-6-$ 


\section{UniversitÀ DEgLi STUdi di FirenZe 80 ANNIVERSARIO 1924-2004}

1. L'Università degli Studi di Firenze 1924-2004. Atti della tavola rotonda di presentazione del volume (Firenze, 17 Dicembre 2004), a cura di Sandro Rogari, 2005

2. Computers, Literature and Philology CLiP 2003. Atti del convegno (Firenze, 4-5 dicembre 2003), a cura di Carlota Nicolás Martínez, Massimo Moneglia, 2005

3. Le lauree honoris causa per gli 80 anni dell'Università degli Studi di Firenzea cura di Sandro Rogari, 2005

4. Le grandi infrastrutture. Approcci di ordine giuridico, economico ed estimativo. Atti del XXXIV incontro di studio del Ce.S.E.T. (Firenze, 15-16 ottobre 2004), a cura di Enrico Marone, 2005

5. L' Università degli Studi di Firenze fra istituzioni e cultura nel decennale della scomparsa di Giovanni Spadolini. Atti del convegno di Studi Firenze, 11-12 ottobre 2004, a cura di Cosimo Ceccuti, Sandro Rogari, 2005

- Le manifestazioni per gli 80 anni dell'Ateneo Fiorentino: eventi e programmi, a cura di Sandro Rogari, 2005 


\title{
L'INNOVAZIONE PER LO SVILUPPO LOCALE
}

\author{
L'UNIVERSITÀ PER IL TERRITORIO
}

ATTI DEL CONVEGNO DI STUDI

EMPOLI, 12 MARZO 2004

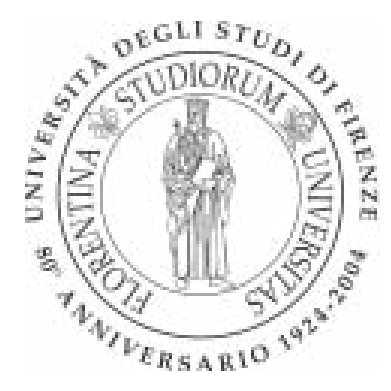

a cura di

Alfiero Ciampolini

Firenze University Press

2005 
L'innovazione per lo sviluppo locale : l'Università per il territorio : atti del convegno di studi : Empoli, 12 marzo 2004 / a cura di Alfiero Ciampolini. - Firenze : Firenze university press, 2005.

(80. anniversario 1924-2004 / Università degli Studi di Firenze, 6) http://digital.casalini.it/8884533627

Stampa a richiesta disponibile su http://epress.unifi.it

ISBN 88-8453-362-7 (online)

ISBN 88-8453-363-5 (print)

378.25094551 (ed. 20)

Firenze - Università - Empoli

(C) 2005 Firenze University Press

Università degli Studi di Firenze

Firenze University Press

Borgo Albizi, 28

50122 Firenze, Italy

http://epress.unifi.it/

Printed in Italy 


\section{Sotto l'Alto patronato del Presidente della Repubblica Carlo Azeglio Ciampi}

\section{Comitato d'onore}

Marcello Pera - Presidente del Senato della Repubblica

Pier Ferdinando Casini - Presidente della Camera dei Deputati

Letizia Moratti - Ministro dell'struzione, dell'Università e della Ricerca Scientifica

Romano Prodi - Presidente della Commissione Europea

\section{Comitato promotore}

Augusto Marinelli - Rettore Università degli Studi di Firenze

Claudio Martini - Presidente della Regione Toscana

Michele Gesualdi - Presidente della Provincia di Firenze

Leonardo Domenici - Sindaco di Firenze

Gianfranco Venturi - Presidente della Provincia di Pistoia

Renzo Berti - Sindaco di Pistoia

Daniele Mannocci - Presidente della Provincia di Prato

Fabrizio Mattei - Sindaco di Prato

Vittorio Bugli - Sindaco di Empoli

Alfiero Ciampolini - Circondario Empolese Valdelsa

Gian Valerio Lombardi - Prefetto di Firenze

Leopoldo Di Mattia - Prefetto di Pistoia

Giuseppe Badalamenti - Prefetto di Prato

Cesare Angotti - Direttore Generale dell'Ufficio Scolastico Regionale per la Toscana

Alberto Carmi - Presidente dell'Ente Cassa di Risparmio di Firenze

Aureliano Benedetti - Presidente della Cassa di Risparmio di Firenze

Giuseppe Mussari - Presidente della Fondazione Monte dei Paschi di Siena

Paolo Mottura - Presidente della Banca Toscana

Ivano Paci - Presidente della Fondazione Cassa di Risparmio di Pistoia e Pescia

Gianni Zonin - Presidente della Cassa di Risparmio di Prato

Luca Mantellassi - Presidente della Camera di Commercio Industria Artigianato Agricoltura di Firenze Andrea Gualtierotti - Presidente della Camera di Commercio Industria Artigianato Agricoltura di Pistoia Luca Rinfreschi - Presidente della Camera di Commercio Industria Artigianato Agricoltura di Prato

Francesco Adorno - Presidente dell'Accademia Toscana di Scienze e Lettere "La Colombaria"

Francesco Sabatini - Presidente dell'Accademia della Crusca

Franco Scaramuzzi - Presidente dell'Accademia dei Georgofili

Giorgio Van Straten - Sovrintendente del Teatro Comunale

\section{Comitato organizzatore}

Sandro Rogari - Presidente - Delegato del Senato Accademico

Paolo Citti

Luigi Lotti 
Enti sostenitori

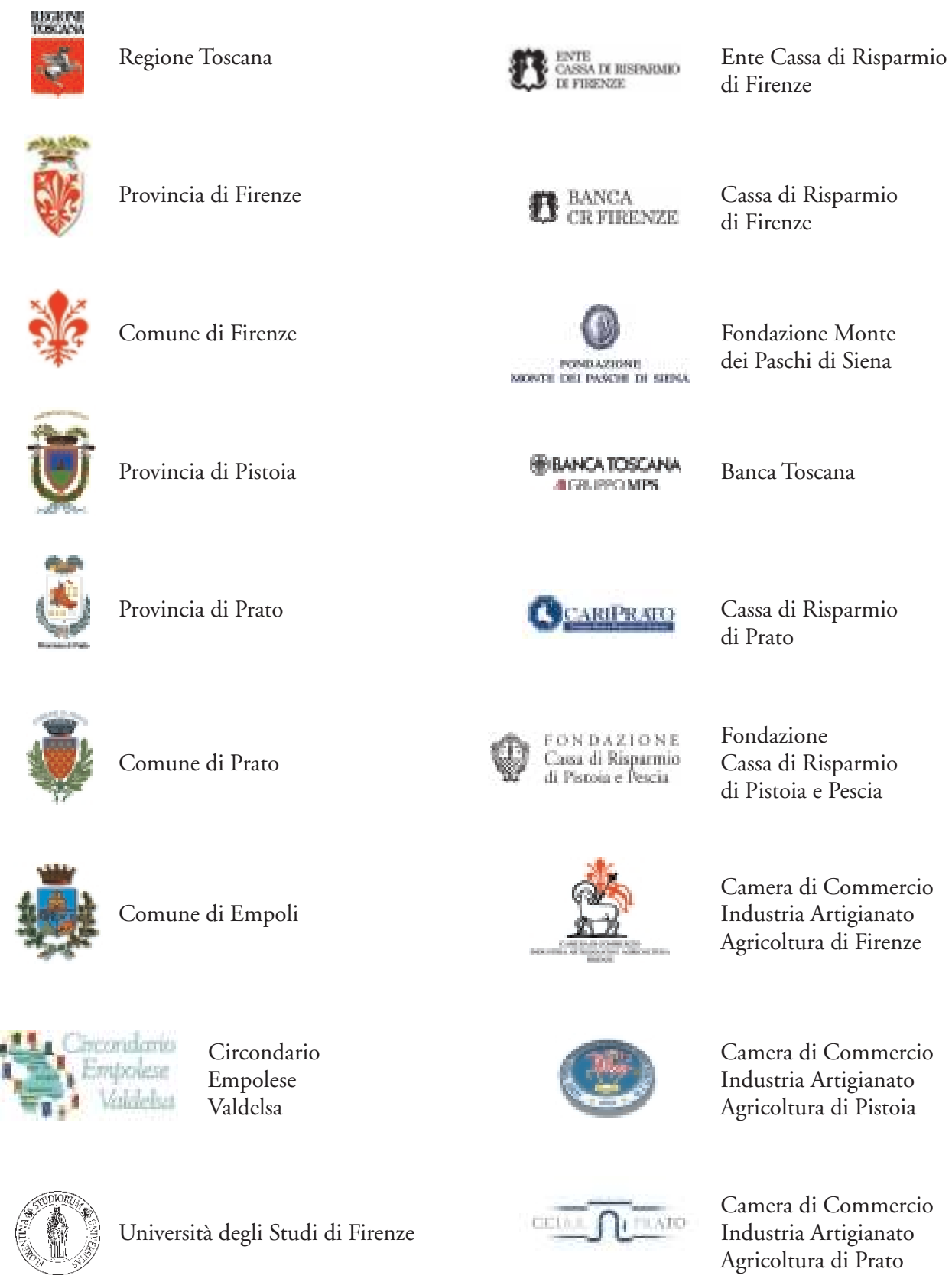

Si ringraziano gli Enti sostenitori delle manifestazioni per gli 80 anni dell'Università degli Studi di Firenze che contribuiscono a sostenere gli oneri finanziari delle pubblicazioni di questa collana. 


\section{Indice}

\author{
Alfiero Ciampolini \\ Luciana Cappelli \\ Vittorio Bugli \\ Enrico Rossi \\ Mario Tucci, Filippo Visintin
}

Iacopo Bernetti

Giancarlo Paba

Pier Angelo Mori

Piero Frediani

Silvano Abati, Emilio Borchi, Alessandro Farini

Antonio Conti, Donatella Lippi

Alberto Magnaghi

Marco Massa
1 Nota del curatore

3 Presentazione

5 Saluto

9 Saluto

11 Il tessuto produttivo empolese: verso quale innovazione tecnologica ed organizzativa?

21 La valorizzazione del patrimonio territoriale per la produzione durevole di nuova ricchezza

29 Territorio, ricerca sul campo e progettualità sociale nei corsi di urbanistica e di pianificazione territoriale del polo universitario di Empoli

33 Competitività e innovazione nelle piccole e medie imprese

41 Il ruolo del chimico per la sicurezza ambientale

53 L'eredità di Leonardo: una ricchezza per l'ottica di oggi

63 La formazione sanitaria ad Empoli da Vincenzio Chiarugi ad oggi

73 Il Polo universitario empolese e le sue prospettive

83 Il programma di sviluppo del Polo universitario e il riuso dell'ex ospedale di San Giuseppe

Indice 
I

IL

7 


\section{Nota del curatore}

Il 12 marzo 2004 si è tenuto presso la Sala Teatro "Il Momento" di Empoli il convegno dedicato a Linnovazione per lo sviluppo locale: l'Università per il territorio, del quale vengono di seguito riportati gli Atti.

Hanno portato il saluto inaugurale al convegno il Presidente del Comitato Organizzatore degli Ottant'anni dell'Università di Firenze, Sandro Rogari, anche a nome del Magnifico Rettore, Augusto Marinelli; il Sindaco di Empoli e Presidente del Circondario Vittorio Bugli e l'Assessore Regionale al Diritto alla Salute Enrico Rossi.

Il convegno è stato realizzato in collaborazione con il Circondario Empolese Valdelsa grazie al contributo dell'Ente Cassa di Risparmio di Firenze, della Banca di Credito Cooperativo di Cambiano e della Fondazione Cassa di Risparmio di San Miniato.

Il convegno ha inteso approfondire gli aspetti pregnanti della presenza dell'Università di Firenze nel territorio empolese-valdelsa che vede impegnate le Facoltà di Agraria, Architettura, Economia, Ingegneria, Medicina e Chirurgia e Scienze Matematiche Fisiche e Naturali.

Tale presenza risponde a un disegno di progressivo consolidamento dell'Università nella vasta area metropolitana fiorentina secondo logiche di insediamento funzionali e connesse alle specifiche esigenze economiche e sociali del territorio.

Il convegno si è concluso con una tavola rotonda ove sono stati rappresentati tutti gli Enti economici e culturali del territorio e con l'inaugurazione della mostra Verso l'atlante del Territorio che si è tenuta nel Palazzo Comunale.

Si ringraziano vivamente tutti coloro che come membri del Comitato Scientifico e del Comitato Organizzatore delle manifestazioni per gli Ottant'anni dell'Università di Firenze, come relatori al convegno e come suoi sostenitori ne hanno permesso la realizzazione.

Empoli, settembre 2005

A.C. 
I

IL

7 


\section{Presentazione di Luciana Cappelli ${ }^{*}$}

Il tema assegnato a questo Convegno, "L'innovazione per lo sviluppo locale: l'Università per il territorio", rappresenta di per sè il significato strategico che abbiamo inteso assegnare alla scelta di dare vita reale al Polo Universitario di Empoli.

Sia l'Università che il Circondario hanno inteso investire energie e risorse nell'ambito di un disegno capace di condensare insieme la crescente domanda di formazione qualificata e l'innovazione che sale dal nostro territorio con l'applicazione, da parte dell'Università, della ricerca mirata alla valorizzazione delle risorse complessive presenti nell'intera area del Circondario.

La scelta, dunque, non trae origine dall'esigenza di un mero decentramento di Corsi di Laurea, quanto da un vero e proprio investimento indirizzato a contribuire alla qualità dello sviluppo locale, partendo dal valore aggiunto che le tante risorse locali possono determinare. Tutt'altro che casuale è stata, a questo proposito, l'individuazione dei Corsi di Laurea, che abbiamo selezionato tenendo presente il loro potenziale ancoraggio con le esigenze specifiche del nostro territorio.

Abbiamo lavorato per costituire un Polo capace di generare uno scambio virtuoso fra quanto può ricevere investendo sulle risorse presenti sul territorio e quanto può restituire, mettendosi a disposizione come motore privilegiato di sviluppo locale.

E' stata una scommessa particolarmente impegnativa per i Comuni del Circondario; adesso si tratta di consolidarla in via definitiva, operando le opportune verifiche e gli eventuali aggiustamenti che l'esperienza maturata consiglia.

Verifiche che, naturalmente, non dovranno prescindere da un ampio coinvolgimento delle più varie istituzioni locali, operanti nei più diversi ambiti: economico, sociale, formativo, culturale.

Nel frattempo stiamo proseguendo nell'impegno di mettere a disposizione strutture adeguate e funzionali, a partire dalla sede unificata nei locali dell'ex ospedale di Empoli, dove sono in corso i primi lavori di ristrutturazione.

Insomma, abbiamo avviato un percorso che dobbiamo condurre a compimento, con la fiducia di poter contare sul contributo e sulla collaborazione sia del contesto locale, che di quello a scala più ampia: provinciale e regionale.

* Sindaco di Empoli e Presidente del Circondario Empolese Valdelsa. 
Le riflessioni e i contributi propositivi che hanno caratterizzato le numerose relazioni del Convegno che presentiamo, costituiscono un indubbio punto di riferimento per il nostro operare, prossimo e futuro.

Anche per questo, non possiamo che essere grati a tutti i protagonisti di questo fecondo appuntamento, organizzato nell'ambito delle manifestazioni degli 80 anni dell’Università degli Studi di Firenze. 


\section{Saluto di Vittorio Bugli*}

Vorrei partire da una considerazione: siamo un'area, siamo un'entità articolata di storia amministrativa e politica, di valori e di culture, di fatti concreti e di consistenze materiali, che è il modello in sè di quello che sempre con maggiore frequenza viene messo un po' in tutti i discorsi, il territorio. Mentre il mondo cambia bisogna renderci conto di cosa cambia a noi, qui, nelle nostre città e nelle nostre case.

Quello che sembra accadere è che il territorio paradossalmente, nel processo di mondializzazione dell'economia acquisti una importanza maggiore rispetto al passato e subisce delle trasformazioni di ruolo che per la loro delicatezza sono paragonabili a quelle delle grandi trasformazioni storiche. Il territorio si trasforma da semplice "contesto" entro il quale avviene e si organizza il ciclo produttivo in "soggetto" della produzione.

L'intero repertorio delle risorse sociali viene quasi a svolgere il ruolo di "materia prima".

Come rendersi praticamente consapevoli di questo? Come agire?

Da questo quadro emergono le grandi sfide che avremo da giocare come territorio.

È con questa consapevolezza del significato che è venuta e viene ad assumere la parola "territorio" che abbiamo giocato in questi anni le nostre sfide principali.

La prima è stata ed è quella del livello istituzionale. Un territorio che voglia dare completezza al suo ruolo necessita di un livello istituzionale proprio. In questo senso la battaglia con la successiva affermazione del Circondario e ora il suo ulteriore rafforzamento.

La seconda, anch'essa già messa in campo, è quella nel senso dell'allargamento dell'orizzonte verso il resto della Toscana: così come le città si aprono all'area, è l'area che si apre alla Toscana. L'obiettivo consapevole è quello dello sviluppo della nostra regione.

La terza è quella di mettersi in grado di non subire l'effetto di un parallelismo che è impellente e dirompente: mentre si mondializza l'economia si economicizza il mondo.

$\mathrm{O}$ saremo in grado, come comunità territoriale, di intervenire direttamente nei processi di formazione e strutturazione degli aggregati sociali o correremo il rischio di un utilizzo indiscriminato, quasi colonizzato del territorio per interessi che risiedono fuori dal territorio stesso e daglia ambiti della decisione democratica.

* Sindaco di Empoli e attuale Presidente della Commissione Attività produttive del Consiglio Regionale della Toscana, è stato Presidente del Circondario Empolese Valdelsa fino al giugno 2004. 
La formazione sarà una delle risorsa principali su cui contare, per il ruolo che ricopre di prima interconnessione tra la produzione di sociale e lo sviluppo economico.

È questa la prima grande scommessa che ci attende: una programmazione dell'istruzione scolastica superiore che sia efficace dal punto di vista del Circondario, una programmazione dell'attività formativa che veda la creazione della nuova classe dirigente, il sapere come costruzione della comunità.

L'Università come punto qualitativo più alto in questa direzione, non tanto e non solo per la presenza dell'università sul territorio, ma per il legame che questa presenza sul territorio consente e consentirà di avere fra società e saperi alti.

Il modello al quale stiamo lavorando è un polo universitario dove si possa scommettere, l'Università possa scommettere, non tanto perché qui siamo in grado di mettere un qualcosa in più (tecnologie, quantità di risorse, di sedi, di dimensione urbana, di peso anagrafico-gravitazionale) ma perché qui siamo in grado, per la nostra storia , per nostra costituzione, per il progetto di società futura che cerchiamo di portare avanti, siamo in grado di mettere a valore con più convinzione e più efficacia i nostri "saperi contestuali" e le nostre risorse specifiche.

Un polo universitaro locale ove le tipologie didattiche ( e di ricerca) siano connesse alle specificità dell'ambiente socio-economico e turistico locale. Un sistema dai contenuti didattici complessi e "localmente orientati".

Un Polo che abbia importanti ricadute in termini di creazione di "centralità urbana", e di innovazione nel sistema economico locale.

Un Polo che sia connesso in rete con tutti i nodi dell'Ateneo per fruire dei saperi dell'intero Ateneo, e viceversa che consenta all'Ateneo di arricchire il proprio patrimonio e la propria peculiarità culturale alimentandosi con gli apporti del nostro polo territoriale.

Un Polo che, sul piano sociale ed economico, possa utilmente appoggiarsi su forme di partnership pubblico-privato che rafforzano la coerenza interna del sistema locale e, al tempo stesso, producono un importante radicamento ed innalzamento culturale opposto ai fenomeni di "migrazione culturale" e di abbandono degli studi che una sede universitaria "remota" induce sugli utenti di aree periferiche.

I processi e le scelte di sviluppo locale possono così appoggiarsi a "competenze" in grado di operare coerentemente rispetto alle caratteristiche, problematicità e possibilità dei diversi sistemi territoriali.

La creazione di un "polo universitario" in questo contesto può essere oggi uno dei motori qualificati di uno sviluppo fondato sulla valorizzazione di queste peculiarità del patrimonio (con l'arco vasto di competenze disciplinari previste dal polo universitario) e contribuire alla crescita delle attività terziarie di servizio indotte dalla messa in valore del patrimonio e dalla cornplessificazione merceologica del sistema produttivo che ne consegue.

Ecco perché le scelte su cui ci siamo orientati guardano ai temi "territorio-aziendaambiente-socio/sanitario".

Non so se tutti questi indirizzi saranno attivati o se ne saranno attivati anche altri qui non presenti, so soltanto che saluto con soddisfazione la possibilità di poter essere a questo punto del percorso per far crescere il nostro territorio. 
Infine, la scelta del Circondano di utilizzare per il polo universitario e i suoi servizi edifici della città storica (l'ex Convento degli Agostiniani, l'ospedale, gli edifici aggregati alla stazione ferroviaria) è destinata a produrre qualificazione funzionale e culturale della città di Empoli che deve svolgere questo ruolo se vuole qualificare la sua capacità di fare "effetto città" nell'interesse di tutto il Circondario.

Dobbiamo avere coraggio e investire con convinzione su questa fondamentale "ipoteca al futuro". E motivare le persone che ne fanno e ne faranno parte perchè si sentano parte di noi.

Ringrazio tutti coloro che finora hanno contribuito a che questo si realizzi e fino da adesso sono convinto che in un futuro non lontano esso sarà uno dei patrimoni di questa comunità. 
I

IL

7 


\section{Saluto di Enrico Rossi ${ }^{*}$}

L'intero sistema socio-economico della nostra Regione è in questi anni impegnato in una sfida senza precedenti: quella di saper coniugare, pena un inevitabile declino e tenendo conto delle compatibilità finanziarie disponibili, sviluppo e qualità. E' su questo terreno che la Giunta Regionale sta giocando la sua scommessa, in un difficile equilibrio di scelte che hanno nell'innovazione il loro fulcro e nel radicamento territoriale una storica ragion d'essere.

In questo quadro il ruolo dell'Università riveste una particolare importanza. In un momento di grandi trasformazioni e pur scontando notevoli difficoltà, spesso di origine nazionale, il sistema formativo e della ricerca della nostra regione sta interpretando con molto impegno la sua parte. La sua presenza articolata conferisce al territorio in cui opera un indiscutibile valore aggiunto. Questo vale per ogni settore di intervento e in modo diretto anche la sanità. Noi crediamo profondamente, ad esempio, nell'integrazione delle articolazioni aziendali del Servizio sanitario toscano con l'Università: la nascita delle aziende ospedaliere universitarie e dei dipartimenti integrati sta a dimostrarlo. Così come la programmazione per aree vaste che stiamo realizzando, un modo coerente e ordinato di guardare alle peculiarità del territorio, tenendo conto nello stesso tempo dell'intera realtà regionale e delle sue relazioni interregionali. La valorizzazione, infine, delle nuove generazioni e delle loro energie al servizio della comunità locale nei termini più avanzati e qualitativamente elevati possibile rappresenta un altro obiettivo del nostro lavoro.

Con questo spirito ci siamo ritrovati ad Empoli per l'appuntamento di cui questo volume rende opportuna testimonianza e con questi intenti continuiamo e continueremo ad operare.

*Assessore Regionale per il diritto alla salute. 
I

IL

7 
Ciampolini A. (a cura di). L'innovazione per lo sviluppo locale ISBN 88-8453-362-7 (online) (C) 2005 Firenze University Press

\title{
Il tessuto produttivo empolese: verso quale innovazione tecnologica ed organizzativa?
}

\author{
di Mario Tucci, ${ }^{*}$ Filippo Visintin ${ }^{*}$
}

\section{Considerazioni generali SUlLe REALTÀ Distrettuali E SUlLE LORO EVOLUZIONI}

Essendo responsabili del 45\% del PIL nazionale e del 39\% dell'occupazione (fonte: «Il Sole 24 Ore», 17 dicembre 2004) con un totale di 239mila aziende, i distretti industriali costituiscono uno dei motori, probabilmente il più importante, dell'economia italiana. Per quanto vasto ed eterogeneo sia l'insieme dei sistemi distrettuali (il sito www.clubdistretti.it individua circa 100 distretti sul territorio nazionale) ed ampio lo spettro dei beni prodotti (ceramiche, tessuti, abbigliamento, pelletteria, sedie, scarpe, minuteria metallica, mattonelle, automobili, mobili, gioielli, ecc.) i distretti sono contraddistinti da alcune caratteristiche comuni che hanno contribuito in larga misura a determinarne il successo, tra queste: i) la presenza, in un ambito territoriale definito e circoscritto, di un numero elevato di imprese, solitamente di dimensioni medio piccole, operanti nello stesso settore e specializzate in singole fasi del processo produttivo; ii) la diffusione, a livello locale, di un forte senso di appartenenza ed un legame molto stretto col territorio, di competenze, know how e di un grande spirito di imprenditorialità; iii) l'esistenza di fitte reti di relazioni [Bellandi, 1982: 355-375] verticali (tra clienti e fornitore), orizzontali (tra imprese operanti nello stesso stadio di produzione) ed anche 'diagonali' tra le imprese produttrici e le imprese fornitrici di macchinari e soprattutto servizi (consulenza, progettazione, trasporto, ecc.).

L'operare in un contesto concorrenziale di imprese condividenti una stessa cultura e fortemente interconnesse tra di loro, sia per quanto riguarda gli aspetti manifatturieri che commerciali, ha portato al conseguimento di notevoli economie non direttamente riconducibili alle singole imprese ma al distretto nel suo insieme [Bombardiere, 2004] e dovute a: i) una riduzione dei costi di transazione: informazioni largamente condivise a livello territoriale, rapporti fiduciari [Dei Ottati, 1987] di lunga durata ed un elevato numero di interazioni, disincentivano comportamenti scorretti ed opportunistici e quindi limitano i costi di monitoraggio, coordinamento e controllo; ii) una riduzione dei costi di produ-

\footnotetext{
* Professore Ordinario di “Gestione degli Impianti Industriali”, Vice Presidente del Corso di Studi in Ingegneria Gestionale.

** Dottorando di Ricerca in "Ingegneria Industriale e dell'Affidabilità".
} 
zione: l'elevata specializzazione delle singole imprese e la relativa facilità con cui queste riescono a coordinarsi ed interagire ha dato origine a sistemi produttivi dotati di notevole efficienza tecnica, flessibilità ed elasticità; iii) allo sviluppo/diffusione di innovazione di tipo incrementale/imitativo attraverso una rapida e fattiva circolazione delle idee; iv) una buona visibilità sul piano internazionale, favorita anche da iniziative di marketing territoriale operato dalle istituzioni locali. L'insieme di questi fattori ha determinato a lungo il successo anche su mercati internazionali dei distretti italiani.

I repentini e radicali cambiamenti verificatisi nel contesto competitivo, a partire dagli anni novanta hanno provocato tuttavia un significativo indebolimento dei sistemi distrettuali, che si trovano oggi a fronteggiare un'allarmante perdita di competitività [Consolati, 2004]. La concorrenza, soprattutto in settori maturi e labour intensive, dei cosiddetti 'paesi emergenti' (oggi diventati potenze economiche di primissimo piano, uno su tutti: la Cina) con manodopera a basso costo e leggi molto più permissive riguardo alle tematiche ambientali ed alla tutela dei lavoratori; l'Euro, che ha limitato la possibilità di utilizzare il tasso di cambio come leva competitiva interna; una dinamica dei tassi di cambio euro/dollaro penalizzante l'esportazioni; ed infine la saturazione dei mercati occidentali, hanno portato infatti ad un drammatico calo della domanda, che costringerà i distretti (se non vorranno scomparire: vedi l'esperienza dei distretti tessili inglesi di inizio novecento) ad evolvere seguendo fondamentalmente due direttrici: l'innovazione e l'internazionalizzazione.

La necessità di sottrarsi a rovinose price competition, che vedrebbero le imprese distrettuali sconfitte in partenza, impone di proporsi sul mercato con beni fortemente differenziati.

Differenziare, in estrema sintesi, significa fare in modo che i consumatori riconoscano al prodotto qualità uniche e quindi lo preferiscano ad altri indipendentemente dal prezzo [Grant, 1991]. Questo è possibile introducendo con regolarità sul mercato prodotti di qualità e caratteristiche superiori (innovazione tecnologica) e cercando, laddove sia possibile, di dotare gli stessi di attributi simbolici (es. marchi) o servizi complementari a corredo (es. assistenza pre e post-vendita) che contribuiscano a renderli difficilmente imitabili.

Mentre sul fronte dell 'innovazione tecnologica di processo, finalizzata all'aumento dell'efficienza dei processi produttivi, ma anche e soprattutto ad un miglioramento dei livelli qualitativi dei prodotti (innovazione di prodotto di tipo incrementale), i sistemi distrettuali sono sempre stati attivi ed abili nello sviluppare soluzioni vincenti [Zagnoli, 1993], appare scarsa la capacità di proporsi sul mercato con prodotti dai contenuti fortemente innovativi (innovazione di prodotto di tipo radicale). L'innovazione tecnologica di prodotto richiede infatti, oggi, competenze (ad esempio nel campo dell'elettronica digitale e dell'informatica) anche molto distanti da quelle storiche che hanno contribuito all'affermazione dei distretti e che difficilmente le PMI distrettuali potranno sviluppare o acquisire all'esterno autonomamente. Le dimensioni ridotte, infatti, se da un lato comportano dinamismo, flessibilità ed elasticità, dall'altro comportano difficoltà di accesso al credito, difficoltà nel finanziare attività costose e rischiose come quelle di ricerca e sviluppo, scarso potere contrattuale nell'attingere al mercato del lavoro e limitate risorse da investire in formazione.

Sono pertanto necessari nuovi paradigmi organizzativi (innovazione organizzativa) che, senza compromettere l'agilità che contraddistingue le PMI, consentano loro, di 'fare sistema' attraverso la creazione di reti o gruppi di imprese (si osservi che a tale proposito 
la governance padronale tipica di queste imprese rappresenta un ostacolo endogeno di notevole entità). Il raggiungimento di una 'massa critica' ed una maggiore condivisione degli orientamenti strategici consentirebbe da un lato, di conseguire economie di scala (nelle attività di ricerca e sviluppo, nelle attività promozionali, negli approvvigionamenti e nella fruizione di servizi comuni) e dall'altro di godere di maggiore forza e rappresentatività anche per aprirsi verso l'estero in maniera sistematica e strutturata.

Per quanto i sistemi distrettuali risultino fortemente internazionalizzati in termini di mercati di sbocco e volume di esportazioni, lo sono assai meno per quanto riguarda gli investimenti diretti all'estero. Sino ad oggi si è assistito prevalentemente all'allestimento di uffici di rappresentanza e piccole filiali commerciali e, eventualmente, ad un decentramento produttivo finalizzato esclusivamente al contenimento dei costi. Questo, oltre a non tener in debita considerazione il concetto di responsabilità sociale verso il sistema distrettuale, ha provocato un impoverimento del territorio e la diffusione di competenze distintive, un tempo esclusive, rivelandosi spesso un danno per i distretti stessi. Per quanto una delocalizzazione delle 'fasi povere' sembri essere invitabile e necessaria, sono auspicabili nuove e più illuminate strategie per accedere a mercati ed ai 'luoghi eccellenti', in cui prendono forma le nuove idee tecnologiche o di business attraverso investimenti diretti ed accordi (partnership commerciali, alleanze strategiche, technology transfer, partecipazioni strategiche, joint venture, ecc.) caratterizzati da intensi flussi informativi, logistici e di risorse umane in vari stadi della catena del valore.

Il distretto non deve più essere inteso quindi come la sede entro cui si sviluppa l'intero processo produttivo, quanto piuttosto come un nucleo di competenze ed abilità sedimentate a livello territoriale ancora indispensabili per presidiare delle funzioni strategiche (tecniche, progettuali, di marketing) in grado di interagire (sfruttando al meglio le opportunità offerte dalla logistica e dall'ICT) con l'esterno, ed in particolare con l'estero, in modo da sfruttare i vantaggi comparati che di volta in volta si presentano.

Questo percorso di crescita, basato sull'innovazione e l'internazionalizzazione, tuttavia, rischia di essere precluso alle realtà distrettuali se esse vengono lasciate a se stesse, non disponendo, queste, delle risorse umane e dei mezzi finanziari per intraprenderlo autonomamente, soprattutto laddove manchino aziende capofila di dimensioni maggiori che possano fungere da traino. Risulta pertanto fondamentale l'intervento di soggetti istituzionali che dovranno: i) promuovere l'immagine dei distretti all'esterno attraverso iniziative di marketing territoriale su vasta scala; ii) intraprendere iniziative per avvicinare le imprese alla conoscenza dei mercati esteri, operandosi nella ricerca dei contatti e delle informazioni preliminari necessarie per poter avviare prima delle politiche di penetrazione commerciale e poi di eventuale partnership; iii) creare i presupposti in termini infrastrutturali affinché possano svilupparsi nuove e più evolute organizzazioni; iv) favorire l'accesso al credito di soggetti di piccola dimensione sviluppando strumenti finanziari adeguati; v) formare risorse umane in grado di interpretare ed affrontare le sfide che il panorama competitivo pone.

Quello della formazione appare come un nodo particolarmente critico soprattutto nelle realtà distrettuali in quanto essa, a tutti i livelli, è basata quasi sempre sul learning by doing. Se da un lato questo ha portato ad un'acquisizione di competenze di notevole importanza e ad un'ottima conoscenza del business è indubbio che, ad oggi, siano 
richieste competenze (in campo tecnologico, informatico, manageriale, giuridico, linguistico) che difficilmente possono essere apprese semplicemente con la pratica.

\section{IL DISTRETTO EMPOLESE-VALDELSANO}

Il tessuto industriale empolese-valdelsano è costituito prevalentemente da piccole e medie imprese ed imprese artigiane operanti in vari settori: tessile/abbigliamento, prodotti in cuoio e lavorazioni delle pelli/calzaturiero, ceramica, agroalimentare e recentemente anche il turismo. Si presenta come una realtà particolarmente dinamica caratterizzata dalla nascita continua di piccole imprese e da un elevato turn-over. Questo, se da un lato è stato fonte di grande vitalità, raramente ha portato alla nascita di soggetti industriali medio-grandi dotati di elevata visibilità sul mercato ed in grado di proporsi come global competitor su scala internazionale.

L'attività manifatturiera è senz'altro la più rilevante (30\% PIL, 35\% UL [IRPET, 2002]) ed è trainata dal 'sistema moda' nelle componenti dell'abbigliamento e della lavorazione dei prodotti in pelle.

\begin{tabular}{|c|c|c|c|c|c|c|}
\hline \multicolumn{7}{|l|}{ CIRCONDARIO DI EMPOLI } \\
\hline & $\begin{array}{l}\text { Area Urb. } \\
\text { Empolese }\end{array}$ & $\begin{array}{r}\text { Bassa Val } \\
\text { d'Elsa }\end{array}$ & Toscana & $\begin{array}{l}\text { Area Urb. } \\
\text { Empolese }\end{array}$ & $\begin{array}{r}\text { Bassa Val } \\
\text { d'Elsa }\end{array}$ & Toscana \\
\hline AGRICOLTURA + IND. ALIMENTARE & 1521 & 1844 & 1386 & 4 & 5,5 & 3,5 \\
\hline \multirow{2}{*}{$\begin{array}{l}\text { INDUSTRIA } \\
\text { di cui }\end{array}$} & 11524 & 10632 & 9304 & 30,1 & 31,7 & 23,6 \\
\hline & \\
\hline tessile ed abbigliamento & 2509 & 2027 & 1872 & 6,6 & 6 & 4,7 \\
\hline \multirow{2}{*}{$\begin{array}{l}\text { prodotti in cuoio, pelletterie } \\
\text { prodotti chimici e sin. }\end{array}$} & 2645 & 3130 & 885 & 6,9 & 9,3 & 2,2 \\
\hline & 850 & 283 & 545 & 2,2 & 0,8 & 1,4 \\
\hline lav. di minerali non met. & 1814 & 795 & 762 & 4,7 & 2,4 & 1,9 \\
\hline macchine ed apparecchi & 918 & 1525 & 1316 & 2,4 & 4,5 & 3,3 \\
\hline \multirow{2}{*}{$\begin{array}{l}\text { COSTRUZIONI } \\
\text { SERVIZI privati }\end{array}$} & 1556 & 1576 & 1764 & 4,1 & 4,7 & 4,5 \\
\hline & 18474 & 14445 & 19849 & 48,3 & 43 & 50,3 \\
\hline \multirow{3}{*}{$\begin{array}{l}\text { commercio e riparazione di aut. } \\
\text { alberghi e ristoranti } \\
\text { trasporti ed attività connesse }\end{array}$} & 6108 & 4928 & 5643 & 16 & 14,7 & 14,3 \\
\hline & 649 & 761 & 1871 & 1,7 & 2,3 & 4,7 \\
\hline & 1996 & 1556 & 2720 & 5,2 & 4,6 & 6,9 \\
\hline Serv. alle imprese e locaz. & 7467 & 5262 & 7016 & 19,5 & 15,7 & 17,8 \\
\hline SERVIZI pubblici & 5165 & 5079 & 7164 & 13,5 & 15,1 & 18,2 \\
\hline TOTALE & 38241 & 33576 & 39466 & $100 \%$ & $100 \%$ & $100 \%$ \\
\hline
\end{tabular}

Figura 1 - Valore aggiunto per settore (dati in milioni di lire relativi all'anno 2000, fonte: IRPET, 2002).

\begin{tabular}{|c|c|c|c|c|c|c|}
\hline \multicolumn{7}{|l|}{ CIRCONDARIO DI EMPOLI } \\
\hline & $\begin{array}{l}\text { Area Urb. } \\
\text { Empolese }\end{array}$ & $\begin{array}{r}\text { Bassa Val } \\
\text { d'Elsa }\end{array}$ & Toscana & $\begin{array}{l}\text { Area Urb. } \\
\text { Empolese }\end{array}$ & $\begin{array}{r}\text { Bassa Val } \\
\text { d'Elsa }\end{array}$ & Toscana \\
\hline AGRICOLTURA + IND. ALIMENTARE & 20 & $\begin{array}{r}29 \\
29\end{array}$ & 20 & 4,5 & 6,2 & 4,4 \\
\hline 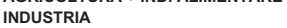 & 167 & 183 & 111 & $\begin{array}{r}4,0 \\
36,9\end{array}$ & 39,4 & 24,4 \\
\hline di cui & & & & & & \\
\hline tessile ed abbigliamento & 58 & 42 & 29 & 12,9 & 9,1 & 6,5 \\
\hline prodotti in cuoio, pelletterie & $\begin{array}{l}50 \\
45\end{array}$ & 64 & 15 & $\begin{array}{r}2,9 \\
9,9\end{array}$ & $\begin{array}{l}9,1 \\
13,7\end{array}$ & $\begin{array}{l}0,5 \\
3,3\end{array}$ \\
\hline prodotti chimici e sin. & 6 & 2 & 4 & 1,4 & 0,5 & 0,8 \\
\hline lav. di minerali non met. & 18 & 13 & 9 & 3,9 & 2,7 & 1,9 \\
\hline prodotti in metallo & 4 & 10 & 10 & 1 & 2,1 & 2,1 \\
\hline mezzi di trasporto & 1 & 0 & 4 & 0.3 & 0,1 & 0,9 \\
\hline macchine ed apparecchi & 10 & 17 & 14 & 2,1 & 3,6 & \\
\hline $\begin{array}{l}\text { COSTRUZIONI } \\
\text { COPPAIECIII }\end{array}$ & 25 & 31 & $\begin{array}{l}14 \\
28\end{array}$ & 5,6 & $\begin{array}{l}3,6 \\
6,6\end{array}$ & 6,2 \\
\hline SERVIZI privati & 161 & 144 & 186 & 35,5 & 31 & 40,9 \\
\hline commercio e riparazione di aut. & 80 & 67 & 75 & $\begin{array}{l}17,5 \\
17,7\end{array}$ & 14,3 & 16,5 \\
\hline alberghi e ristoranti & 16 & 21 & 32 & 3,5 & 4,4 & \\
\hline trasporti ed attività connesse & 20 & 19 & 27 & 4,4 & 4.1 & 5.9 \\
\hline Serv. alle imprese e locaz. & 34 & 29 & 40 & 7,6 & 6,3 & 8,7 \\
\hline SERVIZI pubblici & 79 & 78 & 110 & 17,5 & 168 & 24,2 \\
\hline TOTALE & 452 & 465 & 454 & $100 \%$ & $100 \%$ & $100 \%$ \\
\hline
\end{tabular}

Figura 2 - Unità di lavoro per settore ogni 1000 abitanti (dati relativi all'anno 2000, fonte: IRPET, 2002). 
La plurisettorialità, unitamente alla mancanza sostanziale di connessioni tra le varie attività distrettuali, da un lato ha reso il circondario meno vulnerabile alle crisi dei singoli settori, ma dall'altro ha inibito la creazione di un 'sistema distrettuale' e questo, alla luce delle considerazioni fatte, potrebbe rappresentare in futuro un pesante handicap.

Più che un vero e proprio distretto, quello empolese, si configura infatti come un'aggregazione di imprese più o meno specializzate tra le quali non sussistono sostanziali sinergie che, anche a causa dell' assenza di soggetti di grande dimensione, trova difficoltà ad avere una sufficiente visibilità. I soggetti economici sembrano ancora non aver pienamente compreso le dinamiche dell'attuale contesto competitivo. Questo fatto è testimoniato anche dall'atteggiamento riguardo ai temi dell'innovazione e formazione: da un'indagine svolta dall'IRPET [2002], è emerso come le aziende dell'Area Empolese lamentino una carenza di offerta di lavoro non intellettuale (colmata dall'immigrazione), ma non manifestino interesse alcuno verso l'eventuale assunzione di laureati. La conseguenza di questo atteggiamento è la 'emigrazione' dei giovani il cui livello di scolarizzazione è elevato, verso zone limitrofe (ad esempio l'Area Fiorentina). Questo fatto avrà un effetto nefasto sulla competitività futura delle imprese stesse che, rinunciando a personale con una formazione di alto livello, probabilmente si precluderanno anche la possibilità di crescere ed ammodernarsi.

Istituzioni locali e associazioni imprenditoriali, dovranno quindi adoperarsi sia per rafforzare la coesione del distretto e promuoverne l'immagine, che per sensibilizzare le imprese alle tematiche dell'innovazione ed internazionalizzazione. A questo proposito sono lodevoli le iniziative del Circondario Empolese Valdelsa per la pianificazione e promozione dello sviluppo territoriale attraverso la formulazione di piani locali di sviluppo e la volontà dello stesso di portare sul territorio una realtà universitaria.

\section{IL RUOLO DELL'UNIVERSITÀ. Il CORSO DI LAUREA IN INGEGNERIA Gestionale}

Solitamente la necessità di innovare viene ravvisata al momento in cui si registra una perdita di competitività. Questa perdita di competitività può derivare da problematiche di natura commerciale o da gap tecnologici: nel primo caso si rende necessario un intervento sui processi e, più in generale, sull'organizzazione dell'azienda e delle sue reti di relazioni (innovazione organizzativa); nel secondo caso è necessario acquisire le competenze e le tecnologie necessarie a colmare il gap (innovazione tecnologica) [Campodall'Orto, Vercesi, 2002]. Molto spesso le PMI non dispongono internamente delle risorse per fronteggiare (e spesso neppure per decifrare) questo tipo di situazioni che, con una certa inerzia, subiscono passivamente. In questo contesto la presenza sul territorio dell'Università, ed in particolare della Facoltà di Ingegneria, può comportare notevoli benefici. Una struttura universitaria, da un lato forma risorse umane in grado di interpretare e gestire le problematiche relative all'innovazione e dall'altro può fungere da partner dell'impresa nell'attività di R\&S essendo oltretutto in Italia, il principale soggetto a svolgere tale attività. Collaborazioni tra imprese ed Università 
risultano essere particolarmente fruttuose per entrambe; consentono, infatti, alle prime, l'acquisizione di nuove competenze (in campo informatico, normativo, manageriale e scientifico in senso lato) che difficilmente, e sicuramente a condizioni molto più onerose, potrebbero sviluppare al loro interno; ed alle seconde di trovare importanti sbocchi applicativi ai risultati dell'attività di ricerca. Questi partenariati, inoltre, facilitano ad entrambi l'accesso ai finanziamenti regionali, nazionali ed europei per la ricerca e possono costituire per le aziende un osservatorio privilegiato per il reclutamento, qualora nell'ambito di suddette collaborazioni partecipino, attraverso tesi e tirocini, anche studenti.

\section{L'INGeGneria GeSTIONALE}

Da diversi anni in vari atenei italiani, per formare risorse umane in grado di misurarsi con le problematiche sin qui evidenziate, è stato introdotto un nuovo corso di laurea: l'Ingegneria Gestionale.

La formazione dell'ingegnere gestionale associa competenze economiche e organizzative alla cultura tradizionale dell'ingegnere, di tipo tecnico-industriale. È quindi una figura creata per gestire i sistemi produttivi e logistico-commerciali nelle loro componenti fisiche, informative e organizzative e per promuoverne e gestirne l'innovazione. Oltre a conoscere in modo approfondito gli aspetti teorico-scientifici della matematica e delle altre scienze di base, questi possiede competenze nel campo dell'impiantistica, della logistica, della qualità, della sicurezza, delle tematiche ambientali, dei sistemi informativi ed è in grado di affrontare i temi propri dell'economia aziendale e della gestione d'impresa con l'approccio sistemico e modellistico tipico dell'ingegnere.

Il percorso formativo del Corso di Studi di Ingegneria Gestionale si articola in una laurea di primo livello ed in una laurea specialistica (che a seguito della riforma in approvazione verrà definita Laurea Magistralis). Nella prima vengono affrontate le discipline di base comuni a tutti gli indirizzi e, ad un primo livello di dettaglio, le discipline tipiche dell'Ingegneria Gestionale (economia ed organizzazione aziendale, logistica industriale, gestione della produzione e dei sistemi logistici, impianti industriali, gestione degli impianti industriali, affidabilità e controllo qualità, ecc.) fornendo contenuti professionalizzanti; nella seconda vengono sviluppati ed arricchiti gli strumenti metodologici/analitici ed affrontate in maggior dettaglio le discipline caratterizzanti.

A differenza di altre lauree specialistiche, quella in Ingegneria Gestionale si pone come laurea interdisciplinare, in grado di completare la preparazione di ingegneri provenienti oltre che dalla classe dell'Ingegneria Industriale anche dalle classi Civile e dell'Informazione. Vengono così forniti gli strumenti teorici e le capacità pratiche per svolgere ruoli nei quali le competenze tecniche progettuali sono solo la base necessaria di conoscenza sui processi e sui prodotti, la cui realizzazione dovrà essere garantita gestendo al meglio tutte le risorse, tecniche e umane, di produzione. 


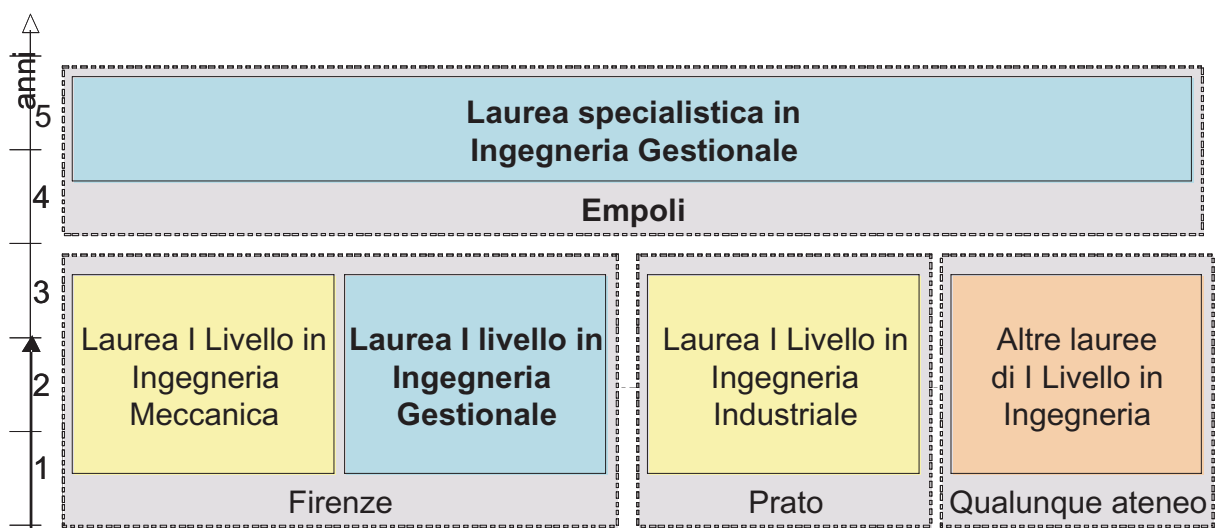

Figura 3 - Interdisciplinarietà del corso di Ingegneria Gestionale.

Gli sbocchi professionali tipici per i laureati specialisti della classe sono quelli dell'innovazione e dello sviluppo della produzione, della progettazione avanzata di processi e sistemi, dell'organizzazione, pianificazione e controllo della produzione, della logistica e del marketing, del project management e controllo di gestione; in sintesi quelli della progettazione e gestione di sistemi complessi, sia nelle imprese manifatturiere o di processo, che nei servizi. Oltre agli sbocchi nell'industria e nel terziario in ruoli di responsabilità operativa e gestionale, il laureato specialista potrà operare anche in diversi ambiti libero-professionali dell'Ingegneria con spiccate competenze progettuali e di analisi da spendere sia all'interno di società di ingegneria e di consulenza, che in forma privata.

\section{OrganizZaZione}

La Facoltà di Ingegneria, in accordo con il Circondario, ha deciso di investire sulla creazione di un presidio locale del Corso di Laurea in Ingegneria Gestionale spostando ad Empoli la laurea specialistica. Le motivazioni di questa scelta sono da ricercarsi nella volontà di non duplicare il corso di base, già attivo su Firenze, e al contempo creare un corso che, vista la sua interdisciplinarietà, sia in grado di attirare studenti provenienti da vari corsi di Ingegneria anche al di fuori dell'area empolese e/o fiorentina.

Per radicare maggiormente il corso di laurea evitando il fenomeno del 'mordi e fuggi' tipico delle sedi decentrate, per il quale i docenti si limitano ad erogare i corsi senza presidiare l'area, sono stati creati due posti di docente a contratto che sono stati assegnati con apposito bando ed attivati nel dicembre 2003, ai quali sono stati affidati compiti rivolti sia agli studenti:

- coperture di alcuni insegnamenti caratterizzanti la laurea specialistica;

- attività di orientamento per iscrizione e ricostruzioni di carriera (si tratta di un compito molto oneroso che è conseguenza della flessibilità voluta con la legge di riforma per le lauree specialistiche); 
- tutoraggio d'aula;

- presidio della sede universitaria di Empoli;

che al territorio:

- gestione dei rapporti con le aziende per l'organizzazione degli stage degli studenti all'ultimo anno;

- individuazione delle esigenze formative e di ricerca per le aziende del Circondario;

- proposta e partecipazione a ricerche di base e applicate con ricadute sul tessuto produttivo del Circondario;

- istituzionalizzazione di rapporti di convenzione fra l'Università e le aziende del Circondario per attività di ricerca e consulenza;

- coordinamento di progetti di ricerca finanziabili dagli organismi pubblici (Regione, Ministeri, Unione Europea, ecc.) che vedano fra i partner qualificati l'Università e aziende, enti o organizzazioni imprenditoriali del Circondario.

Visto il successo riscosso dal corso ed il conseguente numero di iscritti (peraltro in costante aumento) sarà improrogabile la necessità, dal prossimo anno, di una struttura dedicata. Fino ad oggi, dato il transitorio di avvio e l'esiguo numero di iscritti, per altro quasi tutti lavoratori, sono state usate le strutture dell'agenzia formativa del Circondario. Dal prossimo anno accademico è necessario rendere operativa una struttura con almeno due aule da 50 posti. La struttura, anche per motivi di immagine dell'ateneo, dovrebbe essere dedicata esclusivamente ai corsi di laurea e, per raggiungere gli standard minimi per una sede universitaria, dovrà essere dotata servizi di supporto quali la segreteria, una biblioteca e un'attrezzata aula informatica (come peraltro è accaduto per altre realtà decentrate dell'ateneo come Prato e Pistoia).

In tal modo sarà possibile radicare ulteriormente nel territorio il corso di studi $\mathrm{e}$ passare dalla fase sperimentale alla maturità dell'iniziativa.

\section{BibLIOGRAFIA}

Bellandi, M. (1982), Il distretto industriale in Alfred Marshall, "L'Industria», 3, lugliosettembre, I.

Bombardiere, S. (2004), Un approccio sistemico al distretto industriale: ipotesi e riflessioni, paper interno, Dipartimento di Scienze della Gestione d'Impresa, Università "La Sapienza”, Roma.

Campodall'Orto, S., Vercesi, P. (2002), Deve l'università occuparsi di trasferimento tecnologico?, "Mondo Digitale», dicembre.

Consolati, L. (2004), Quale futuro per i distretti italiani?, <http://www.mglobale.it/>, 10 dicembre.

Dei Ottati, G. (1987), Il mercato comunitario, in Becattini, G. (a cura di), Mercato e forze locali: il distretto industriale, Il Mulino, Bologna.

18 L'INNOVAZIONE PER LO SVILUPPO LOCALE

Mario Tucci, Filippo Visintin 
Grant, R.M. (1991), Contemporary Strategy Analysis. Concepts, Techniques, Applications, Blackwell, Oxford.

IRPET (2002), Piano Locale di Sviluppo Circondario Empolese Valdelsa: indirizzi strategici e quadro analitico.

Zagnoli, P. (1993), Percorsi di diversificazione dei distretti industriali: il caso Prato, Giappichelli, Torino. 
I

IL

7 


\title{
La valorizzazione del patrimonio territoriale per la produzione durevole di nuova ricchezza
}

\author{
di Iacopo Bernetti*
}

\section{Il RUOLO DELLA SPERIMENTAZIONE DIDATTICA E DELLA RICERCA E LE Ricadute sul territorio del Circondario Empolese Valdelsa}

\subsection{Verso un nuovo modello di sviluppo territoriale}

Il significato sociale ed economico delle risorse agroforestali e ambientali, ed in particolare del settore rurale ha avuto in questi ultimi anni una profonda evoluzione, con un conseguente altrettanto rilevante mutamento nelle esigenze di governo del territorio. Infatti l'affermazione di modelli ad industrializzazione diffusa, il maggior ricorso al pendolarismo, la sempre minore coincidenza fra esodo agricolo ed esodo rurale ed i fenomeni di controesodo hanno contribuito ad interrompere le relazioni fra rurale ed urbano in termini di soli trasferimenti, per stabilire uno stato di crescente interdipendenza funzionale fra queste due realtà.

L'evoluzione quindi del territorio agroforestale da contesto disagiato a risorsa da tutelare impone alle attività produttive agricole una responsabilità di salvaguardia ecologica, con uno sviluppo economico del mondo rurale sostenibile dal punto di vista sociale e ambientale. Questo principio si traduce a livello soprattutto aziendale nella evoluzione delle attività produttive con una maggior valorizzazione del territorio, intendendo con esso le risorse naturali, gli aspetti storici artistici e soprattutto il tessuto sociale con le sue tradizioni produttive che spesso si identificano con un 'sapere territoriale'.

A livello istituzionale questo nuovo modo di indirizzare il governo del territorio agroforestale rilancia l'esigenza di recuperare la cosiddetta 'arte della localizzazione' riconducendo ad un unico disegno di sviluppo le problematiche di recupero, conservazione e valorizzazione del patrimonio storico, artistico e naturale.

Per le aree rurali caratterizzate da un'agricoltura di qualità, sia in termini di prodotti sia di paesaggio, un modello di sviluppo capace di interpretare le specificità locali coerentemente ai principi della sostenibilità può essere quello di tipo 'dal basso, endogeno e autocentrato'.

"Professore Ordinario di Economia ed Estimo rurale, Corso di laurea triennale in Urbanistica e Pianificazione Territoriale e Ambientale. 
Con il concetto di 'sviluppo dal basso' si intende la promozione del processo di sviluppo da parte di una comunità intesa come insieme di soggetti economici, politici e culturali. Tale principio è formalizzato da Friedman e Weaver [1979] attraverso il concetto di «unità di integrazione territoriale» definita come la sovrapposizione e quindi la collaborazione fra spazi sociali, economici e politici. Ognuna di queste unità di integrazione territoriale esprimerebbe secondo gli autori una propria domanda di autonomia nell'intraprendere processi autonomi di sviluppo.

Lo 'sviluppo autocentrato' è invece un processo di sviluppo sulla valorizzazione delle risorse interne attraverso una specializzazione del processo produttivo volta a realizzare prodotti con una specifica identità territoriale.

Il quadro teorico di tali approcci non è tuttora completamente sistematizzato, ma si basa su un insieme eterogeneo di principi ispiratori che possono essere così sintetizzati [Conti, 1996].

Ogni comunità localizzata territorialmente possiede una dotazione di risorse di tipo ecologico, storico e culturale che rappresentano un vero e proprio 'potenziale endogeno' che trova la massima possibilità di valorizzazione in processi di sviluppo integrati. Definire con precisione l'entità del potenziale endogeno di un territorio montano è compito arduo: esso corrisponde all'idea che si fanno di un territorio coloro che cercano un margine, una leva per promuovere il processo di sviluppo. Il concetto coinvolge:

- le risorse naturali e culturali, materiali (flora, fauna, beni storici ed architettonici) e immateriali (tradizioni, cultura locale);

- le risorse umane, cioè la popolazione che risiede sul territorio e le istituzioni, con gli impegni che gli amministratori locali sono portati ad assumersi;

- le attività e le imprese (con la loro concentrazione geografica di distretto e filiera), i mercati e l'immagine che il territorio riesce a promuovere.

I processi di sviluppo dal basso e autocentrato si basano sulla valorizzazione delle potenzialità endogene attraverso percorsi che permettano di mantenere o di acquisire una vera e propria 'competitività territoriale' in grado di affrontare la concorrenza sul mercato tramite la promozione della qualità ambientale come elemento distintivo del territorio e la collaborazione e concertazione fra le componenti sociali, economiche e politiche.

L'attività di sperimentazione didattica e di ricerca svolta nell'ambito del CDL di Urbanistica e Pianificazione Territoriale ha tentato di concretizzare questo indirizzo di governo del territorio attraverso la sperimentazione didattica incentrata nell'ambito del territorio del Circondario Empolese Valdelsa. Nell'anno in corso tale impostazione si è esplicata nel laboratorio "Il Montalbano: una nuova via per lo sviluppo sostenibile".

Il laboratorio rappresenta una forma di attività didattica interattiva, da svolgersi in aule attrezzate, pluri-disciplinari, che comprende:

- attività didattiche frontali di carattere teorico-metodologico di supporto all'attività progettuale;

- attività analitico-progettuali del laboratorio (in piccoli gruppi con compiti individuali) applicate a casi di studio seguite dai docenti e dai tutor. 


\subsection{Il laboratorio "Il Montalbano: una nuova via per lo sviluppo sostenibile"}

\subsubsection{Le risorse sociali}

Il Montalbano rappresenta per le sue caratteristiche un campo di studio particolarmente interessante per l'analisi delle risorse sociali. Dal punto di vista delle comunità, sono infatti presenti istituzioni pubbliche sensibili ai problema riguardanti la sostenibilità dello sviluppo e numerose associazioni locali impegnate nella valorizzazione del territorio, disposte ad orientare la riflessione e gli indirizzi di sviluppo verso nuovi indirizzi strategici.

Mettere a sistema questi attori sociali ha voluto dire selezionare le loro istanze ed istituzionalizzarle all'interno del processo di piano: l'incontro di questi attori con il patrimonio può produrre la costruzione di una nuova identità, e quindi dare l'avvio ad un nuovo ciclo di territorializzazione.

Gli attori ed i progetti sociali possono così essere brevemente elencati:

- le rilevanti risorse paesistiche e storico-culturali dell'area possono consentire un'insieme di sinergie fra i comuni in grado di esaltarne la qualità dell'offerta turistica;

- il ruolo, complementare al punto precedente, che può essere svolto da una produzione agricola, che già adesso presenta punte di assoluta eccellenza soprattutto nel settore vitivinicolo ed olivicolo, può consentire una svolta nel trend produttivo attuale ed innescare nuove pratiche agricole che facciano da traino alla nuova territorializzazione del Montalbano;

- alle precedenti attività produttive si affiancano notevoli permanenze storiche che possono consentire di strutturare interessanti filiere con l'attività agrituristica e con la ricettività rurale;

- presenza di attori economici interni ed esterni molto attivi nei campi vitivinicolo, agrituristico, convegnistico, che possono essere interessati ad una rivitalizzazione territoriale del Montalbano;

- delle risorse sociali fanno parte anche le iniziative da parte della amministrazioni locali fortemente indirizzate alla valorizzazione del patrimonio territoriale; esempi di queste iniziative sono: la proposta avanzata dal Consorzio Interprovinciale del Montalbano per la creazione di un Parco Regionale, la costruzione da parte del Circondario Empolese Valdesa della Costituente partecipativa del Circondario e della Provincia di Prato, con una prima esperienza pilota del Progetto Bias per la media Valdibisenzio;

- il progetto di costituzione della strada del Vino, Olio e dei produttori agricoli e agroalimentari di qualità, promosso dal Patto per lo sviluppo del Montalbano;

- "Le Colline di Leonardo", i comuni del Montalbano unitamente alle associazioni di categoria intendono istituire il Comitato Promotore per la costituzione della Strada del Vino e dell'Olio del Montalbano, ponendosi come obiettivi: incremento reddito aziende agricole, rafforzamento aziende esistenti, costituzione nuove aziende (giovani);

- la "strada del Vino, Olio e dei Prodotti agricoli e agroalimentari di qualità del Montalbano" ha come punti di forza: viticoltura, olivicoltura, produzioni tipiche locali e toscane, alta intensità di aziende agrituristiche, consolidamento del 
volume di affluenza turistica nazionale ed estera, posizione strategica, clima mediterraneo, risorse ambientali di pregio; l'obiettivo dell'iniziativa è quello di realizzare una rete di strade che colleghino le località aventi elevata importanza, tenendo in debito conto la panoramicità e il loro stato di manutenzione. La strada del Vino e dell'Olio, unitamente all'esistente Strada Medicea dei Vini di Carmignano, si propone di diventare la spina dorsale di tutto il sistema del Montalbano.

\subsubsection{Le risorse territoriali}

L'elevata qualità del sistema ambientale del Montalbano si può identificare, nella lunga durata, con l'area boschiva sommitale, la media collina del paesaggio agrario storico e la zona umida del sistema ripariale dell'Arno e dell'Ombrone.

La notevole varietà morfologica del sistema orografico (forre, valli, colline sabbiose, dolci dorsali...) contribuisce a creare un ambiente contraddistinto da una media biodiversità, presente soprattutto nelle ampie superfici boscate del Barco Reale.

Risalendo dalle prime pendici fortemente urbanizzate verso la sommità del crinale aumenta la biodiversità e con essa le valenze ecologico-naturalistiche di questo sistema; le pressioni da inquinamento si fanno via via minori.

Coesistono sul Montalbano sia patrimoni vasti e significativi come ad esempio quello delle sistemazioni agrarie storiche, dei ciglioni e dei terrazzamenti (in differenti stati di conservazione), sia delle testimonianze più puntuali, come ad esempio i castelli, le pievi ed i centri storici.

L'estensione delle aree terrazzate è di circa 1050 ettari e rappresenta poco più del $4 \%$ dell'intera area di studio. Il dato quantitativo non esprime la straordinaria importanza, ambientale, storico-culturale e visiva dei terrazzamenti che definiscono, più di ogni altro tipo di sistemazione, l'impronta storica del Montalbano.

I terrazzamenti affondano le loro radici in tempi lontani, ma è certo che il massimo sforzo nella loro diffusione appartiene al periodo sette-ottocentesco che coincide in buona parte con il Granducato dei Lorena.

Il terrazzamento costituisce la manifestazione più laboriosa ed estesa di colonizzazione rurale su terre di per sé poco adatte all'agricoltura: un processo di domesticazione manuale della natura guidato dalla necessità di creare il piano sul pendio. Il vantaggio elementare di separare la terra dalla pietra, accumulando suolo coltivabile rinserrato dentro l'abbraccio dei muri a secco, si unisce all'efficacia idraulica: il terrazzamento è un ottimo sistema drenante perché non trattiene solo la terra con i muri a secco, ma anche l'acqua piovana che viene ceduta lentamente attraverso i muri da un ripiano all'altro invece di scorrere sul pendio dilavandone le superfici.

È impossibile trovare muri a secco molto antichi: come un corpo che rinnova incessantemente le sue cellule, il terrazzamento ha attraversato i secoli con una continua metamorfosi fisiologica interna. Il terrazzamento ha motivazioni funzionali e produttive, ma costituisce nel suo complesso anche una grandiosa operazione di mutamento scenografico del paesaggio originario: ciò gli attribuisce di fatto anche un valore di natura estetica. 
Le perdite di suolo costituiscono una delle principali cause di degradazione della fertilità del suolo: a seguito dell'azione battente delle gocce di pioggia e dello scorrimento superficiale delle acque, viene asportata la parte più superficiale del terreno, quella più ricca di elementi nutritivi e di sostanza organica, dove risiede la maggiore attività microbiologica. Il suolo rappresenta una risorsa non rinnovabile, o rinnovabile in tempi molto lunghi, e come tale deve essere conservata in modo da evitare una progressiva diminuzione della potenzialità produttiva e della sostenibilità economica.

Dobbiamo considerare come patrimonio anche i prodotti tipici che le terre del Montalbano continuano a produrre: i suoi vini soprattutto, poi l'olio, la cui valorizzazione è fondamentale per assicurare il mantenimento del paesaggio, ma anche produzioni più limitate come quella dei fichi di Carmignano, generalmente presenti negli oliveti tradizionali, e della cioccolata di Monsummano.

L'elevata qualità e identità del paesaggio storico è inoltre fortificata dal raffinato sistema insediativo policentrico formato da nuclei urbani storici di elevato valore, dalla rete delle pievi con le loro chiese suffraganee e dalle abbazie collocate sull'importante viabilità di crinale, dalle rocche e dai castelli, memoria di feroci lotte medievali, dal placido sistema delle ville fattoria, delle ville medicee, dei poderi, e dei mulini, dalla piccola rete dei musei che vanno da quello del merletto e del ricamo di Casalguidi a quello etrusco di Artimino.

Questo patrimonio, areale e puntuale, fortemente strutturato e connotato storicamente dalla presenza antropica, frutto dei sedimenti delle civilizzazioni etrusca, medievale, rinascimentale e lorenese, è connesso attraverso il reticolo stradale storico, ancora fortemente riconoscibile.

La valorizzazione di questa viabilità viene letta come opportunità per un recupero di percorsi storici e di connessioni ecologiche in grado di recuperare le relazioni ambientali, culturali e fruitive fra i due patrimoni.

\subsubsection{I progetti diffusi}

Questi capisaldi progettuali sono quelle azioni che vanno ad interessare tutto il territorio del Montalbano e si prefiggono di guidare lo sviluppo generale del territorio.

La loro è un'azione diffusa di cucitura e di valorizzazione fra le varie parti del territorio.

\subsubsection{La valorizzazione del paesaggio in senso esteso (ecologia del paesaggio)}

Mantenere e valorizzare il paesaggio del Montalbano riveste particolare importanza non solo per l'alto valore storico-artistico delle pendici collinari e delle coperture boscate, $\mathrm{ma}$ anche in relazione alle indicazioni contenute nello scenario, che indirizzano le azioni al riconoscimento ed al potenziamento del patrimonio territoriale ed alla sua valorizzazione economica con la crescita del turismo 'sostenibile'.

Gli obiettivi per la valorizzazione del paesaggio sono i seguenti:

- fornire regole che garantiscano un corretto rapporto tra territorio agricolo e relative pratiche d'uso, insediamenti urbani ed elementi naturali; 
- creare una rete di nuove connettività ecologiche su tutto il territorio con corridoi naturalistici di relazione tra tutte le risorse ambientali per consentire la continuità ecologica fra un habitat e l'altro;

- mantenere e recuperare la biodiversità;

- valorizzare i caratteri tradizionali del paesaggio agricolo forestale (coltivi ad olivo e seminativo su terrazzamenti a secco e ciglioni con relativi sistemi di scolo delle acque e manufatti di collegamento; complessità e alternanza delle trame agrarie e delle aree boscate; aree umide lungo i corsi d'acqua; sistemi di ville fattoria collegati alle coloniche ed ai poderi; sistemi insediativi di crinale e di mezza costa articolati in villaggi borghi, case da signore ed edifici rurali sparsi);

- contrastare la semplificazione colturale dovuta all'espansione dell'economia vinicola di alta qualità garantendo l'alternanza di campi a vigneto, oliveto, seminativo, bosco, siepi, viali alberati;

- regolare il recupero ed il mantenimento della trama agraria storica;

- promuovere il turismo rurale ed agrituristico connesso al recupero di edilizia storica;

- recuperare il patrimonio edilizio esistente ed in particolare gli insediamenti rurali come risorsa predominante per soddisfare la domanda residenziale;

- disciplinare interventi al patrimonio edificato attraverso abachi che contengano regole di trasformazione sia per i centri urbani che per l'edilizia sparsa;

- attivare processi di recupero urbanistico dove necessari.

\subsection{Il Laboratorio del "Parco dell'Arno"}

Il laboratorio del Parco dell'Arno, tuttora in corso di svolgimento, riguarda territori dei comuni di Empoli, Vinci, Montelupo Capraia e Limite, collegandosi con il parco fluviale di Lastra a Signa.

Il progetto tiene conto della recente evoluzione delle politiche sui sistemi fluviali (vedasi i "Contratti fiume" europei e il programma dell'Associazione per l'Arno allegato) da politiche di riduzione del rischio (idraulico e inquinologico) a politiche maggiormente attente sia agli aspetti ambientali (il fiume come corridoio ecologico) sia agli aspetti territoriali e fruitivi (praticabilità della riviera e sua percorribilità, piste ciclabili, ippovie, riqualificazione dei fronti urbani rivieraschi, riconnessione della riviera agli spazi pubblici urbani, riconnessione con i percorsi escursionistici, agrituristici ed enogastronomici nel territorio, navigabilità, riqualificazione dei valori paesistici e fruitivi del territorio rurale in relazione al fiume.

Il progetto si qualifica pertanto come multisettoriale integrato e si avvale di diversi contributi disciplinari (urbanistici, idrogeologici, idraulici, storici, paesistici, ecologici, agronomici, forestali).

La valorizzazione degli aspetti ambientali e territoriali del sistema fluviale richiede di selezionare e qualificare le politiche e i progetti di riduzione del rischio idraulico e inquinologico in modo da contribuire in modo multifunzionale all'aumento della qualità ambientale e territoriale. 
I riferimenti geografici per il progetto sono:

- l'intero bacino idrografico nel tratto di riferimento;

- le fasce di pertinenza fluviale (autorità di bacino);

- i sistemi urbani e i sistemi territoriali locali afferenti al fiume;

- i sistemi ambientali;

- i sistemi agriforestali.

Dall'integrazione dei diversi ambiti di riferimento è definita a livello di progetto un corridoio fluviale multifunzionale a 'geometria variabile' che costituisce la base territoriale della perimetrazione del parco e delle aree pre-parco. A sua volta il corridoio fluviale sarà articolato in subsistemi, ovvero tratti di fiume che presentano una tipologia peculiare dal punto di vista della relazione del fiume con il territorio. Es. tratti che percorrono aree agricole, aree boscate, aree urbane, aree miste, aree compromesse, ecc., che consentiranno di trattare in modo peculiare e differenziato le diverse problematiche che concorrono alla definizione del progetto.

Le analisi necessarie per impostare il progetto sono:

- usi storici del fiume e descrizione dell'evoluzione storica del sistema fluviale e del suo territorio;

- usi attuali del fiume e criticità (idrauliche, inquinologiche ambientali, territoriali);

- usi previsti dal progetto e risorse territoriali e ambientali (urbane, infrastrutturali, rurali e forestali) da attivare.

Le analisi settoriali riguardano:

- struttura e funzionamento idraulico del bacino imbrifero; le politiche di settore in progetto (Autorità di Bacino);

- $\mathrm{i}$ livelli inquinologici del fiume e le politiche in atto (ARPAT);

- la struttura e il funzionamento dei sistemi ambientali e le politiche in atto;

- la struttura dei sistemi territoriali e urbani e politiche in atto;

- la struttura del sistema rurale;

- le politiche in atto (di settore e locali, istituzionali e non) sul sistema fluviale.

Il progetto si sta avvalendo di un modello di valutazione polivalente che assume come criterio guida la valutazione di coerenza fra quattro ambiti di azione:

- azioni di riduzione del rischio idraulico;

- azioni di mitigazione del rischio inquinologico;

- azione di riqualificazione dei sistemi ambientali afferenti al fiume;

- azioni di riqualificazione territoriale e urbana per la fruizione del sistema fluviale.

Il modello, che si avvarrà di una griglia di riferimento per l'analisi delle politiche, consentirà:

- di valutare le politiche in atto in relazione al contributo di ciascuna di esse a uno o più ambiti di azione;

- di scegliere azioni settoriali coerenti e in sinergia con gli altri livelli di azione.

La valorizzazione del patrimonio

L'INNOVAZIONE PER LO SVILUPPO LOCALE 


\section{BIBLIOGRAFIA}

Bernetti, I., Fagarazzi, C. (2002), Limpiego dei modelli multicriteriali geografici nella pianificazione territoriale, "Aestimum", 41, dicembre.

Conti, S. (1999), Geografia economica: teoria e metodi, UTET, Milano.

Friedmann, J., Weaver, C. (1979), Territory and Functions. The Evolution of Regional Planning, Arnold, Londra.

Magnaghi, A. (2000), Il progetto locale, Bollati Boringhieri, Torino. 


\title{
Territorio, ricerca sul campo e progettualità sociale nei corsi di urbanistica e di pianifica- zione territoriale del Polo universitario di Empoli
}

\author{
di Giancarlo Paba*
}

1. I corsi di laurea triennale e specialistica del Polo universitario di Empoli nel campo territoriale (Urbanistica e Pianificazione Territoriale e Ambientale; Pianificazione e Progettazione della Città e del Territorio) sono caratterizzati dai seguenti elementi significativi:

- Una particolare concezione del radicamento dell'università nel territorio. In questa concezione il territorio non si limita ad ospitare le strutture universitarie e queste ultime non si limitano a chiedere risorse materiali e organizzative. Il rapporto è viceversa assai più complesso, recuperando una tradizione disciplinare che è possibile far risalire al plannerscozzese Patrick Geddes (nel famoso progetto per un'università in India e nello stesso progetto per l'Università di Gerusalemme). L'università viene infatti concepita come una componente fondamentale del capitale culturale e intellettuale del territorio, una risorsa in senso proprio, risorsa aperta, attiva, inserita in una matrice estesa di connessioni e collegamenti con la comunità insediata, le economie locali, e con la stessa molteplicità di saperi esistenti. Tra università e territorio si istituisce quindi un circuito virtuoso di relazioni e di vantaggi reciproci.

- I corsi a carattere urbanistico e territoriale del polo universitario di Empoli sono inoltre caratterizzati da una particolare impostazione didattica. Il territorio viene considerato come un intreccio complesso e stratificato di componenti geofisiche, ambientali, storiche, socio-economiche, identitarie. Questa concezione, posta alla base dell'impostazione scientifica dei corsi di Empoli, è all'origine di uno sguardo sfaccettato e molteplice che le strutture didattiche (moduli professionalizzanti, corsi e insegnamenti, esercitazioni, laboratori didattici, stage, tesi di laurea) rivolgono al territorio circostante. Il territorio di Empoli e della Valdelsa finisce per diventare una gigantesca aula didattica all'aperto a disposizione dei docenti, degli studenti, delle amministrazioni, della stessa popolazione.

- Infine il piccolo sistema universitario che si è concentrato e insieme ramificato nel polo empolese (abitato oggi da una vera e propria comunità di professori,

\footnotetext{
* Professore Ordinario di Tecnica e pianificazione urbanistica, Presidente del corso di laurea specialistica in Pianificazione e Progettazione della Città e del Territorio.
} 
tecnici, tutor, assegnisti di ricerca, docenti a contratto e giovani ricercatori) ha una concezione del rapporto tra università, ricerca e territorio di tipo attivo, esposto ai problemi sociali e culturali esistenti nel contesto. La ricerca deve quindi essere dentro il territorio, utile e socialmente significativa, aperta ai contributi e alle interazioni con la molteplicità degli attori sociali, degli interessi individuali e collettivi, degli abitanti.

2. In particolare la concezione di ricerca sopra esposta presenti i seguenti caratteri significativi.

- La ricerca è strettamente legata alle istituzioni locali e al sistema di amministrazione e di governo del territorio, sia attraverso rapporti diretti (attraverso una molteplicità di forme che vanno dalla collaborazione volontaria alla firma di vere e proprie convenzioni di ricerca), sia attraverso lo sviluppo dei molteplici fili di interazione che nascono dal funzionamento stesso della piccola macchina universitaria costruita nel corso del tempo (seminari, convegni, mostre ed esposizioni dei lavori didattici e dei prodotti di ricerca, attività culturali).

- La ricerca cerca di costruire il suo percorso di azione entro un rapporto positivo e attivo con la società locale, in particolare attraverso le relazioni con il ramificato tessuto associativo del circondario, con le scuole e soprattutto con le molteplici forme di progettualità sociale e di protagonismo delle associazioni e degli abitanti.

- La ricerca attivata nei corsi di Empoli è orientata al progetto, a un'idea di trasformazione positiva dei territori e degli insediamenti, in modo aperto e a volte persino informale e sperimentale: dalla costruzione di scenari condivisi di trasformazione della società locale, alla collaborazione progettuale verso la risoluzione di piccoli problemi talvolta legati all'esistenza stessa del polo universitario.

3. I campi di ricerca attivati in questi anni sono quindi legati alle caratteristiche del rapporto università, ricerca e territorio sul quale ci siamo soffermati nei punti precedenti. Non è possibile qui riportare in dettaglio un quadro completo delle attività in corso; esse tuttavia possono essere raggruppate nel modo seguente:

- ricerche di carattere teorico e metodologico (sulla rappresentazione del territorio e degli insediamenti, sulle tecniche innovative di rappresentazione delle identità locali, sullo sviluppo locale autosostenibile, sulla concezione del patrimonio territoriale come cardine di una politica alternativa di sviluppo e di gestione delle risorse ambientali, sulla partecipazione e sulle politiche territoriali interattive).

- ricerche indirizzate verso la costruzione di un quadro di conoscenze e di sollecitazione attiva dei processi di autoriconoscimento e autorappresentazione della società locale (atlanti territoriali e socio-economici, costruzione di sistemi informativi territoriali, repertori della progettualità sociale).

- ricerche coordinate con gli altri corsi di laurea (in particolare con i corsi di agraria, ingegneria e economia) orientate verso la costruzione di un quadro multidisciplinare di analisi della società locale. 
- ricerche a contenuto propositivo o esplicitamente progettuale, come quelle sul sistema fluviale dell'Arno e dei suoi affluenti, ma anche quelle che riguardano altri territori della Toscana come le ricerche sul recupero abitativo dei centri storici dell'Amiata o sulla riqualificazione urbanistica dei piccoli centri della pianura pistoiese.

- le ricerche hanno costituito inoltre il supporto di iniziative collaterali di qualche importanza come per esempio il contributo alla creazione della Costituente partecipativa del comune di Empoli, o il supporto scientifico fornito dalla comunità dei docenti di Empoli alla redazione della Carta del Nuovo Municipio e alle attività che, sulla base di quella carta, caratterizzano la Rete del Nuovo Municipio, alla quale le amministrazioni dell'area empolese e la piccola università di Empoli hanno dato un apporto determinante. 
I

IL

7 


\title{
Competitività e innovazione nelle piccole e medie imprese
}

\author{
di Pier Angelo Mori*
}

\section{NUOVI MERCATI E NUOVA CONCORRENZA}

Nell'ultimo scorcio del ventesimo secolo diversi eventi hanno aumentato la concorrenza a livello mondiale e mutato l'allocazione delle produzioni. Il crollo dei regimi dell'est europeo, l'integrazione europea e la liberalizzazione della circolazione di merci, persone e capitali nel mercato unico europeo, la moneta unica, una serie di accordi internazionali di libero scambio e non ultimo le politiche nazionali di tutela della concorrenza, tutto ciò ha significato per l'Italia, soprattutto a partire dagli anni novanta, una maggiore esposizione alla concorrenza europea e mondiale e una maggiore apertura dei mercati interni in settori tradizionalmente protetti o ad accesso regolamentato. In breve, le politiche per la concorrenza da una parte e l'evoluzione dello scenario politicoeconomico mondiale dall'altra hanno congiuntamente contribuito a produrre maggiore concorrenza. La domanda da cui vorrei partire nella mia riflessione è questa: il nuovo contesto che si è creato è favorevole o sfavorevole alla piccola e media impresa (PMI), soprattutto con riferimento a quella operante nel settore manifatturiero?

Tutto ciò che favorisce una maggiore concorrenza sul mercato interno dei prodotti (che per noi è ormai quello europeo) non può che rafforzare la piccola e media impresa nei confronti della grande. Prendiamo la tutela della concorrenza. Per sua natura la PMI normalmente non gode di posizioni di monopolio sul mercato e non ha un potere di mercato rilevante da sfruttare. Pratiche anticoncorrenziali quali le intese collusive e le varie forme di abuso di posizione dominante non sono proprie delle PMI: queste possono semmai essere danneggiate dal potere monopolistico dei concorrenti di maggiori dimensioni e dalle pratiche anticoncorrenziali di questi. La legislazione di tutela della concorrenza quindi non può che avvantaggiare la PMI. Anche l'allargamento del mercato dovuto alla liberalizzazione degli scambi rafforza la posizione della PMI nei confronti di quelle più grandi, sia dove queste competono sullo stesso lato del mercato sia dove la piccola è fornitrice della grande.

Le PMI godono di alcuni vantaggi competitivi sui mercati finali che hanno consentito loro di diffondersi e prosperare. Sui mercati dei beni standardizzati il vantaggio della

* Professore Ordinario di Economia Politica, Università di Firenze. 
piccola dimensione sta nei minori costi fissi dell'organizzazione. Nei mercati di nicchia, sempre grazie alla dimensione, la PMI può effettuare produzioni che alla grande non convengono. L'elevata flessibilità nell'organizzazione della produzione, anche questa in parte dovuta alla dimensione, è un ulteriore vantaggio competitivo. La PMI ha dunque armi e strategie sue proprie per rispondere a aumenti di concorrenza sui mercati dei prodotti, siano essi finali che intermedi.

L'apertura del mercato interno e la maggiore concorrenza sui singoli mercati nazionali offrono dunque alle PMI l'opportunità di rafforzare la propria posizione nei confronti delle imprese domestiche di maggiore dimensione - cioè di imprese che operano in ambienti normativi e tecnologici simili. Quindi, se nel corso del processo gli attori non variano, possiamo affermare che la maggiore concorrenza porta benefici alle PMI. Ma l'allargamento degli sbocchi ha comportato anche l'entrata di nuovi concorrenti di livello basso. Sono questi i concorrenti dei paesi emergenti e dell'est europeo, che possono contare su condizioni ambientali radicalmente diverse da quelle domestiche, con vantaggi di costo tali da annullare i vantaggi competitivi delle PMI interne. Pertanto le PMI esposte alla concorrenza internazionale rischiano oggi di essere schiacciate dalla pressione concorrenziale dei nuovi concorrenti.

Nei settori in cui la pressione dei nuovi concorrenti è rilevante la risposta strategica è principalmente una: spostarsi nella scala della qualità dei prodotti più in alto dei nuovi concorrenti, in modo da collocarsi su mercati che sono fuori della portata di questi (almeno temporaneamente), o spostarsi verso l'alto nella tecnologia di produzione, in modo da compensare con il vantaggio tecnologico gli svantaggi ambientali. In entrambi i casi la chiave di volta della strategia di riposizionamento sul mercato è l'innovazione di prodotto o di processo. Che per rispondere alla nuova concorrenza non siano sufficienti i fattori tradizionali di vantaggio competitivo (i nuovi concorrenti godono di vantaggi ancora maggiori), ma occorra invece innovare, appartiene ormai al senso comune. Ma in questa operazione di riposizionamento sul mercato le PMI indubbiamente si scontrano con alcune difficoltà oggettive, che vorrei rapidamente ricapitolare.

\section{LE DIFFICOLTÀ DI ACCESSO PER LE PMI AI MERCATI DEI FATTORI}

L'innovazione tecnologica dal punto di vista economico può essere rappresentata come un processo di acquisizione di nuovi fattori di produzione della tre specie fondamentali: capitale fisico (ovvero tecnologia), capitale finanziario e lavoro. I tre livelli sono strettamente collegati tra loro. L'acquisizione di nuova tecnologia spesso richiede a valle nuove competenze di lavoro in grado di interagire con essa e a monte conoscenze di supporto all' individuazione, scelta e implementazione della nuova tecnologia. Inoltre questa è tipicamente incorporata in capitale fisico -macchine, impianti, infrastrutture- che richiede investimenti e quindi la disponibilità di capitale finanziario. Dunque per competere sul mercato dei prodotti bisogna accedere ai mercati di questi fattori. Come si posiziona la PMI su essi?

Il piccolo/medio imprenditore è quello che maggiormente si avvicina all'archetipo schumpeteriano dell'imprenditore sprovvisto di risorse proprie, il quale per dare corpo 
alla propria idea imprenditoriale deve necessariamente ricorrere al credito. Nella teoria di Schumpeter è il credito (ovvero la creazione di moneta) il carburante del processo innovativo e in ultima analisi il motore della società capitalistica. Il banchiere è una figura cruciale di questo processo, perché è il vero e proprio produttore (più che l'intermediario $)^{1}$ della moneta che alimenta l'innovazione ed è colui che per conto della società seleziona i progetti imprenditoriali meritevoli di credito. Affinché il processo dell'innovazione tecnologica sia efficiente deve pertanto essere efficiente il circuito del credito. L'efficienza nell'erogazione del credito richiede anzitutto che vi sia trasparenza e uguaglianza di trattamento. In altre parole i progetti per i quali si richiede un credito devono essere valutati per la loro qualità intrinseca (ivi compresa la qualità gestionale garantita dal proponente) e non per aspetti estranei a esso (relazioni personali del proponente, scambi di favori, ecc.). Inoltre il credito che qui interessa in modo particolare è quello accordato all'idea imprenditoriale, non al patrimonio. In altre parole, la funzione socialmente rilevante del credito non è rendere temporaneamente liquidi i patrimoni, ma mettere risorse produttive a disposizione di chi non le ha e invece dispone di un progetto imprenditoriale.

Tutte queste condizioni sembrano lontane dall'essere soddisfatte in Italia. Nonostante i mutamenti che hanno interessato il settore nell'ultimo decennio e che si sono tradotti in un aumento della concorrenza al suo interno, il credito è tuttora estremamente conservatore: molto spesso si concede credito a un patrimonio (portato a garanzia) e non a un progetto imprenditoriale, e questo vale in particolare per la PMI. Si può affermare che la tendenza a concedere credito all'idea imprenditoriale piuttosto che al patrimonio è inversamente proporzionale alla dimensione di impresa: dunque, tanto più piccola l'impresa, tanto più difficile per l'imprenditore giocare il ruolo dell'innovatore schumpeteriano. Da questo punto di vista, nonostante le tante trasformazioni che hanno investito il settore creditizio negli ultimi anni, non si sono fatti grandi progressi e molto resta ancora da fare.

Ma ancor prima di proporre un progetto al banchiere per il finanziamento, l'imprenditore deve aver individuato la nuova tecnologia da adottare. Anche qui la dimensione non gioca favorevolmente. La tecnologia non può essere prodotta né adattata in casa: la PMI non ha la massa critica per creare un surplus sufficiente a fare in proprio un'attività di ricerca e sviluppo (ma potrebbe farla insieme ad altri, come diremo più avanti). Per accedere tempestivamente all'innovazione tecnologica (la tempestività è essenziale, perché la nuova tecnologia è efficace come strumento strategico fintanto che non è accessibile ai concorrenti), bisogna possedere delle conoscenze tecnologiche e canali d'accesso al mercato della tecnologia, che è un mercato mondiale. Anche qui carenze di risorse umane adeguate e una dimensione tradizionalmente localistica della PMI, rendono questo processo di gran lunga più difficile che non per le imprese di maggiori dimensioni.

${ }^{1}$ Schumpeter, J.A., La teoria dello sviluppo economico, Sansoni, Firenze 1971 (tr. it. della $4^{\mathrm{a}}$ ed. ted. di Theorie der Wirtschaftlichen Entwicklung, Duncker und Humblot, Berlino 1934 [I ed. 1912]). 
Quest'ultima osservazione rimanda al terzo problema, forse quello meno evidente, certamente quello meno discusso: le risorse umane a sostegno del processo innovativo. Nuova tecnologia significa oggi nuove competenze professionali. Non è stato sempre così. All'inizio dell'era fordista la nuova tecnologia ha progressivamente incorporato competenze che precedentemente appartenevano al patrimonio professionale del lavoratore e ha reso così possibile il deskilling della forza-lavoro, cioè il ricorso a competenze professionali più basse. Oggi numerosi studi indicano che ci troviamo in una fase diversa, soprattutto a causa della rivoluzione dell' informazione e della comunicazione, una fase in cui la nuova tecnologia sempre più spesso comporta non sostituzione con competenze più basse ma al contrario impone un innalzamento. Ciò richiede il supporto di un'adeguata formazione professionale - l'altra forma di investimento, oltre a quello in capitale fisico, necessario per l'innovazione. Anche a questo riguardo vale quel che vale per la ricerca e sviluppo: la PMI non la può produrre in proprio e quindi è soprattutto fruitrice di formazione prodotta da terzi. I problemi di accesso sono qui in parte diversi che per la ricerca e sviluppo, ma sono ugualmente rilevanti (su questo ritorneremo più avanti).

Tutti e tre i mercati -credito, tecnologia, lavoro qualificato- presentano per la PMI difficoltà oggettive di accesso. Non è questa una novità di oggi: sono problemi storici delle PMI in Italia, rispetto ai quali poco è mutato nel corso degli anni. La vera novità è proprio questa: mentre la posizione delle PMI sui mercati dei fattori non è sostanzialmente mutata, al contrario sono molto cambiati i mercati dei prodotti. Vincoli, strozzature, mancanza di concorrenza sui mercati dei fattori inevitabilmente riducono la competitività delle PMI e quindi la capacità di reggere a una maggiore concorrenza sul mercato del prodotto. Il problema è tutto qui: l'allargamento del mercato dei prodotti non si è accompagnato a un più agevole accesso ai fattori dell'innovazione e, stretta nella morsa tra una nuova concorrenza sul mercato dei prodotti e antiche rigidità sui mercati dei fattori di produzione, non solo la PMI rischia di non poter cogliere le opportunità di sviluppo che l'allargamento dei mercati finali indubbiamente comporta, ma anche di essere espulsa da essi. La PMI investita dalla nuova concorrenza si trova dunque in una fase evolutiva critica, dall'esito incerto: la maggiore concorrenza può alla fine rivelarsi letale anziché benefica per essa.

\section{RicerCA E INNOVAZIONE IN TOSCANA}

Tutto questo vale in generale per le PMI italiane. Ma quali sono la situazione attuale e le prospettive in Toscana? Come si sta effettivamente comportando il sistema toscano nel campo della ricerca e dell'innovazione? Una recente indagine svolta dal Dipartimento di Scienze Economiche dell'Università di Firenze consente di mettere a fuoco questi problemi e di dare alcune risposte. ${ }^{2}$

${ }^{2}$ La ricerca è stata pubblicata in Lombardi, M., Mori, P., Vasta, M. (a cura di), Sistema innovativo e fattori strategici: il caso della Toscana, Franco Angeli, Milano 2003. 
Il sistema della ricerca comprende due categorie principali di attori: centri di ricerca di varia natura e le imprese. In Toscana il problema critico è che i centri di ricerca-prevalentemente rappresentati dalle Università- e le imprese viaggiano su piani e a velocità differenti, con scarsa interazione tra loro. Questo fatto ha rilevanti implicazioni per l'innovazione industriale e la capacità dell'industria toscana di tenere il passo. Ma procediamo con ordine.

Il potenziale tecnico-scientifico di un territorio dipende da una molteplicità di fattori, dalla dotazione di capitale umano alla quantità di risorse dedicate alla ricerca. La nostra ricerca ha calcolato un indicatore sintetico del potenziale (IIR) che tiene conto dei seguenti fattori:

- prodotto interno lordo;

- percentuale di spesa in R\&S finanziata dal settore pubblico sul totale del PIL regionale;

- percentuale di spesa in R\&S finanziata dal settore privato sul totale del PIL regionale;

- tasso di scolarità;

- percentuale di laureati in discipline scientifiche sul totale dei laureati;

- indice regionale delle infrastrutture (calcolato come media ponderata delle infrastrutture esistenti nella regione: rete viaria, ferroviaria ed altri mezzi di trasporto, disponibilità di collegamenti telefonici, qualità e struttura della rete di distribuzione elettrica, ecc.).

I risultati mostrano che la Toscana occupa una posizione di confine tra le regioni a maggiore potenziale innovativo -in linea di massima quelle del Nord, oltre al Lazio-e quelle a minore potenziale, cioè quelle del Centro-Sud e la Liguria (vedi tabella seguente).

Scendendo su un piano più analitico, per quanto riguarda la dotazione di capitale umano la Toscana si colloca tra le regioni italiane con il più elevato tasso di scolarità secondaria superiore ma, al contempo, si caratterizza anche per una presenza di laureati inferiore a quella della maggior parte delle regioni centro-settentrionali. Particolarmente rilevante appare la debolezza nell'istruzione tecnica, sia per il livello secondario che per quello universitario. Queste tendenze sono almeno in parte spiegabili con le caratteristiche della domanda di lavoro espressa da ampi segmenti dell'economia toscana: come evidenziato da diversi studi, le esigenze delle imprese toscane, sia nell'industria che nei servizi, sono centrate su mansioni di carattere esecutivo, per le quali il possesso del diploma secondario risulta più che sufficiente. La ridotta presenza di diplomati in discipline tecniche fa anche ritenere che la debolezza della domanda di lavoro qualificato da parte dell'industria toscana si estenda alle mansioni tecniche intermedie, per lo svolgimento delle quali il possesso di un diploma tecnico non risulta strettamente necessario.

La particolare composizione dell'industria toscana, con una dimensione media delle imprese inferiore a quella nazionale e con una concentrazione nei comparti tradizionali molto più accentuata che a livello nazionale -assai bassa è, ad esempio, la quota di occupazione industriale in imprese science based, che pesa per la metà rispetto al dato 
nazionale- si riflette anche sull'intensità dell'attività di R\&S da parte delle imprese. La quota percentuale sul PIL della spesa in R\&S nel periodo 1992-1996 (periodo cui si riferiscono i dati più recenti) è per la Toscana leggermente inferiore al dato nazionale, ma con una particolarità: l'attività di R\&S risulta fortemente dipendente dal settore pubblico. Un ruolo di primo piano è infatti giocato dal sistema universitario in cui la Toscana ha un peso notevole a livello nazionale, distribuito su una molteplicità di aree di ricerca e particolarmente rilevante proprio nelle discipline tecnico-scientifiche, a cui si contrappone la particolare debolezza degli investimenti aziendali in R\&S, con valori allineati a quelli delle regioni italiane meno sviluppate. Anche nella produzione di bre-

\begin{tabular}{|c|c|c|c|c|}
\hline 1995 & 1996 & 1997 & 1998 & 1999 \\
\hline Lombardia & Emilia-Romag na & Emilia-Romag na & Emilia-Romag na & Emilia-Romag na \\
\hline Emilia-Romagna & Lombardia & Lombardia & Lombardia & Piemonte \\
\hline Piemonte & Piemonte & Piemonte & Piemonte & Lombardia \\
\hline Lazio & Lazio & Veneto & Veneto & Veneto \\
\hline Veneto & Veneto & Lazio & Lazio & Lazio \\
\hline Liguria & Toscana & Toscana & Toscana & $\begin{array}{l}\text { Trentino-Alto } \\
\text { Adige }\end{array}$ \\
\hline Toscana & $\begin{array}{l}\text { Friuli-Venezia } \\
\text { Giulia }\end{array}$ & $\begin{array}{l}\text { Friuli-Venezia } \\
\text { Giulia }\end{array}$ & $\begin{array}{l}\text { Friuli-Venezia } \\
\text { Giulia }\end{array}$ & Toscana \\
\hline $\begin{array}{l}\text { Friuli-Venezia } \\
\text { Giulia }\end{array}$ & Liguria & Liguria & $\begin{array}{l}\text { Trentino-Alto } \\
\text { Adige }\end{array}$ & $\begin{array}{l}\text { Friuli-Venezia } \\
\text { Giulia }\end{array}$ \\
\hline Marche & Marche & $\begin{array}{l}\text { Tre ntino-Alto } \\
\text { Adige }\end{array}$ & Marche & Marche \\
\hline $\begin{array}{l}\text { Trentino-Alto } \\
\text { Adige }\end{array}$ & $\begin{array}{l}\text { Trentino-Alto } \\
\text { Adige }\end{array}$ & Marche & Liguria & Liguria \\
\hline Abruzzo & Abruzzo & Abruzzo & Abruzzo & Abruzzo \\
\hline Umbria & Umbria & Umbria & Umbria & Umbria \\
\hline Puglia & Puglia & Puglia & Sicilia & Sicilia \\
\hline Campania & Sicilia & Sicilia & Puglia & Molise \\
\hline Sicilia & Campania & Campania & Campania & Campania \\
\hline Sardegna & Sardegna & Sardegna & Sardegna & Puglia \\
\hline Molise & Molise & Molise & Molise & Sardegna \\
\hline Calabria & Calabria & Calabria & Calabria & Calabria \\
\hline
\end{tabular}

Ranking delle regioni italiane in base allo IIR, anni 1995-1999 (manca la Basilicata; la Valle d'Aosta è unita al Piemonte). 
vetti la performance regionale non appare complessivamente molto brillante: la Toscana oscilla tra la settima e l'ottava posizione per numero di brevetti ogni 10.000 abitanti. In definitiva, a fronte di numerosi fattori di debolezza, l'unico vero punto di forza del sistema della ricerca tecnico-scientifica in Toscana è la presenza di un'elevata spesa in ricerca da parte del sistema pubblico.

Il quadro della Toscana risulta dunque caratterizzato da un divario tra il potenziale tecnico-scientifico esistente e la capacità di produrre innovazioni tecnologiche in grado di rafforzare la competitività dell'apparato produttivo esistente. Se si esaminano le strutture di ricerca presenti sul territorio (un'ottantina di centri), rispetto a diversi fattori filoni di ricerca, risorse umane e strumentali, strategie- emerge che i punti di forza sono: i) il livello scientifico della ricerca pubblica, che è generalmente buono, con alcune punte di assoluta eccellenza (elettronica, bio-medicale, informatica); ii) le forti relazioni nazionali e internazionali e una buona capacità di attirare fondi per la ricerca, soprattutto pubblici. A fronte di questi abbiamo alcuni punti di debolezza, tra cui il più rilevante è la rigidità nella gestione delle risorse umane che impedisce un veloce aggiornamento del capitale umano, ma nel complesso il livello di performance del sistema toscano della ricerca è di ottimo livello.

Il vero problema è che i due sistemi -centri di ricerca e imprese regionali- sono poco propensi ad interagire l'uno con l'altro: ciascuno dei due sembra cioè percorrere propri sentieri evolutivi che, nel corso degli ultimi anni, si sono probabilmente distanziati ulteriormente. In particolare, dai dati disponibili l'elevata capacità progettuale della ricerca pubblica toscana -in particolare delle Università- non sembra incidere in modo significativo sull'andamento dei principali indicatori di output dell'attività innovativa. Questa discrasia è indicativa di difficoltà nell' istituire solide relazioni tra Università ed impresa, in grado di stimolare la ricerca applicata e l'applicazione nell'impresa della ricerca prodotta all'esterno.

\section{Conclusioni}

I centri di ricerca debbono produrre innovazione tecnologica, ma è poi necessario che le competenze e le conoscenze esistenti siano incanalate verso le imprese: particolarmente carente appare la comunicazione tra ricerca e impresa, nonostante i progressi della società della comunicazione, a cui peraltro fa fronte un'accresciuta complessità della tecnologia. Sono scarsi i momenti di contatto tra imprese e mondo della ricerca, che favoriscano un' interazione anche operativa. Eppure la concentrazione sul territorio toscano di competenze e istituzioni di ricerca offre indubbi vantaggi. La presenza di numerosi centri di eccellenza nella ricerca e nella formazione scientifica e tecnica, Università in testa, comporta la disponibilità di risorse umane competenti nelle tecnologie di punta localizzate sul territorio, facilitando le interazioni.

La vera sfida è quindi aumentare la comunicazione e l'interazione tra le due realtà: gli interventi dovrebbero privilegiare l'area intermedia tra i centri di ricerca e le imprese, senza tuttavia trascurare l'altro fronte, quello dell'innovazione tecnologica prodotta 
internamente all'azienda. A questo proposito è necessario tenere presente soprattutto l'impresa tipica della realtà manifatturiera toscana, cioè quella piccola e media, che non favorisce lo sviluppo della ricerca tecnologica all'interno della realtà aziendale. Questo handicap strutturale può tuttavia essere attenuato mediante interventi che mirino ad incentivare: i) la cooperazione tra imprese, che è la via principale attraverso cui sviluppare la ricerca tecnologica in un contesto di frammentazione delle realtà aziendali; ii) gli spin-off di nuove imprese che, operando in settori congruenti con il potenziale tecnico-scientifico presente a livello regionale, possano non soltanto beneficiare delle condizioni favorevoli sopra delineate ma produrre anche un travaso di conoscenze verso l'intero sistema produttivo.

Tutto ciò richiede anzitutto iniziativa imprenditoriale, ma occorrono anche soluzioni organizzative nuove, funzionali agli obiettivi che si vogliono raggiungere: aggregazioni verticali e orizzontali, temporanee o permanenti per sviluppare quelle competenze professionali di cui la PMI necessita per competere, ma che singolarmente ha difficoltà a procurarsi.

I poteri pubblici non possono sostituirsi ai privati, ma possono aiutare questo processo, a livello finanziario e non solo: le imprese di concerto con i loro organismi di rappresentanza hanno bisogno, oltre che di risorse finanziarie, di norme, di consulenza e anche di canali di trasmissione delle informazioni per potersi aggregare e promuovere appropriate iniziative di formazione e ricerca e sviluppo. Per entrambe la chiave di volta è la cooperazione. Solo cooperando le imprese più piccole possono diventare attori del processo e competere ad armi pari con le imprese più grandi, pur mantenendo la propria originalità: 'cooperare' e 'aiutare a cooperare' sono le parole d'ordine per una nuova stagione di sviluppo delle PMI e del territorio in cui operano. 


\title{
Il ruolo del chimico per la sicurezza ambientale
}

\author{
di Piero Frediani*
}

Nell'Anno Accademico 1996-97 l'Università di Firenze decentrò ad Empoli il corso di Diploma in Chimica e per sostenere questa iniziativa fu costituita l'associazione per il Diploma in Chimica ad Empoli alla quale aderivano l'Università di Firenze, i Comuni dell'area empolese, l'Associazione Industriali Fiorentini, la Camera di Commercio, alcuni Istituti bancari ed altri sostenitori locali. Mentre i primi due anni a carattere propedeutico del corso di Diploma in Chimica venivano tenuti a Firenze, il terzo e conclusivo anno del Diploma veniva tenuto ad Empoli ove venivano insegnate le materie più professionalizzanti del corso stesso. Infatti i corsi del III anno erano in parte affidati a docenti provenienti dalle industrie dell'area empolese in modo da preparare gli studenti ad un rapido inserimento nelle realtà locali.

Con la riforma dell'Università del 1999 (DM n. 509 del 3/11/1999) l'insegnamento è stato modificato come illustrato nella figura 1 .

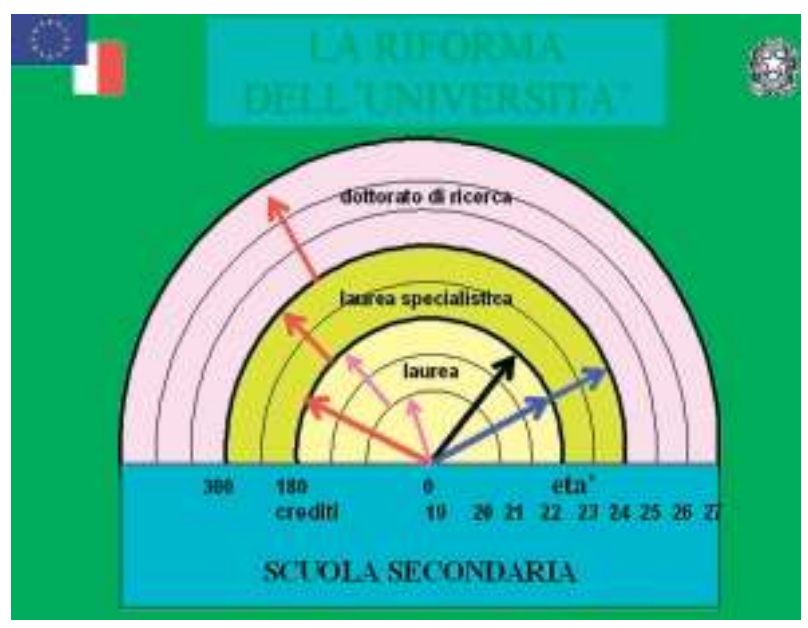

Figura 1 - Organizzazione degli studi universitari dopo la riforma del 1999.

* Professore Ordinario di Chimica industriale, Presidente del CDL Chimica Applicata, Università degli Studi di Firenze. 
Gli studenti provenienti dalle scuole medie superiori possono iscriversi ad una laurea triennale. Al termine gli studenti interessati possono frequentare una laurea specialistica della durata di due anni e successivamente coloro che lo vogliono possono proseguire ulteriormente gli studi frequentando un corso di dottorato della durata di 3 anni. Alla conclusione di questo percorso di studi uno studente ottiene il titolo di dottore di ricerca in Chimica.

Nell'adeguare i corsi di studio alla riforma universitaria del 1999 la Facoltà di Scienze MFN ha deciso di trasformare il corso di Diploma in Chimica nella Laurea triennale in Chimica Applicata che si inserisce nella Classe XXI della riforma universitaria (figura 2).
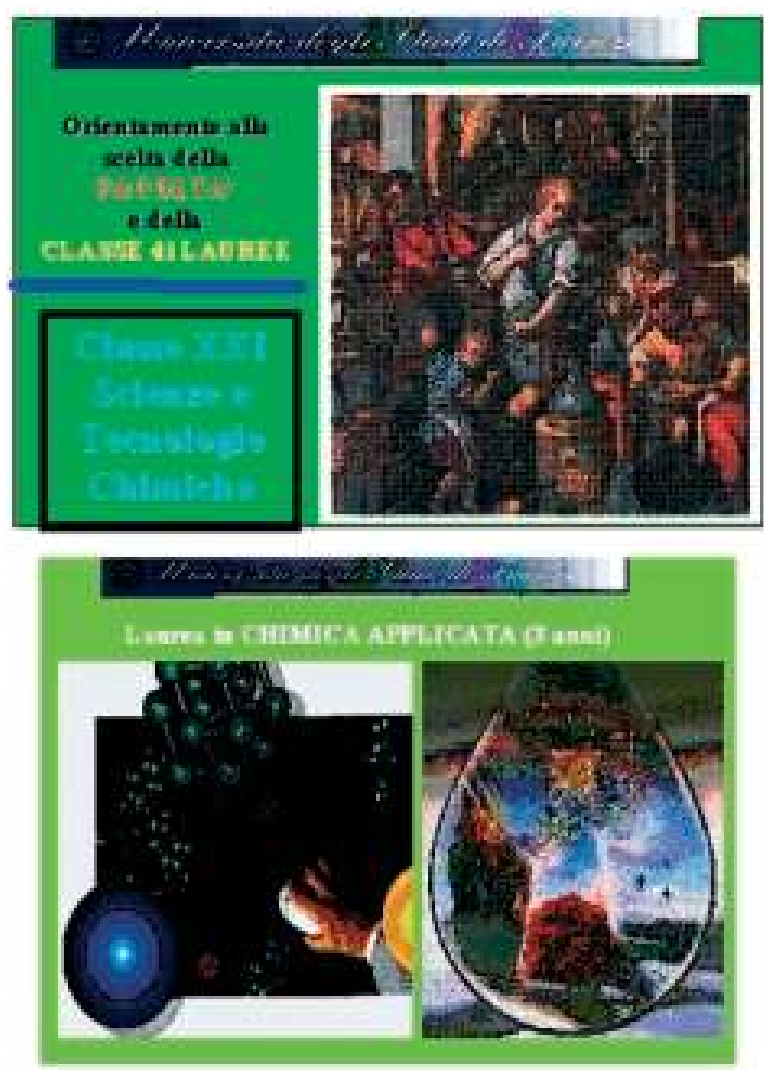

Figura 2 - La laurea triennale in Chimica Applicata nelle nuove classi di laurea.

La corrispondenza tra la laurea triennale in Chimica Applicata ed il precedente corso di Diploma in Chimica è riportata nella figura 3 insieme con il successivo percorso di studio che uno studente ha la possibilità di seguire. Il primo anno del corso di diploma è praticamente rimasto inalterato nella laurea triennale mentre gli altri due anni sono stati in parte modificati per soddisfare le esigenze della legge di riforma universitaria per quanto riguarda il tipo di corsi che devono essere svolti ed il numero dei crediti da attribuire a questi corsi. 


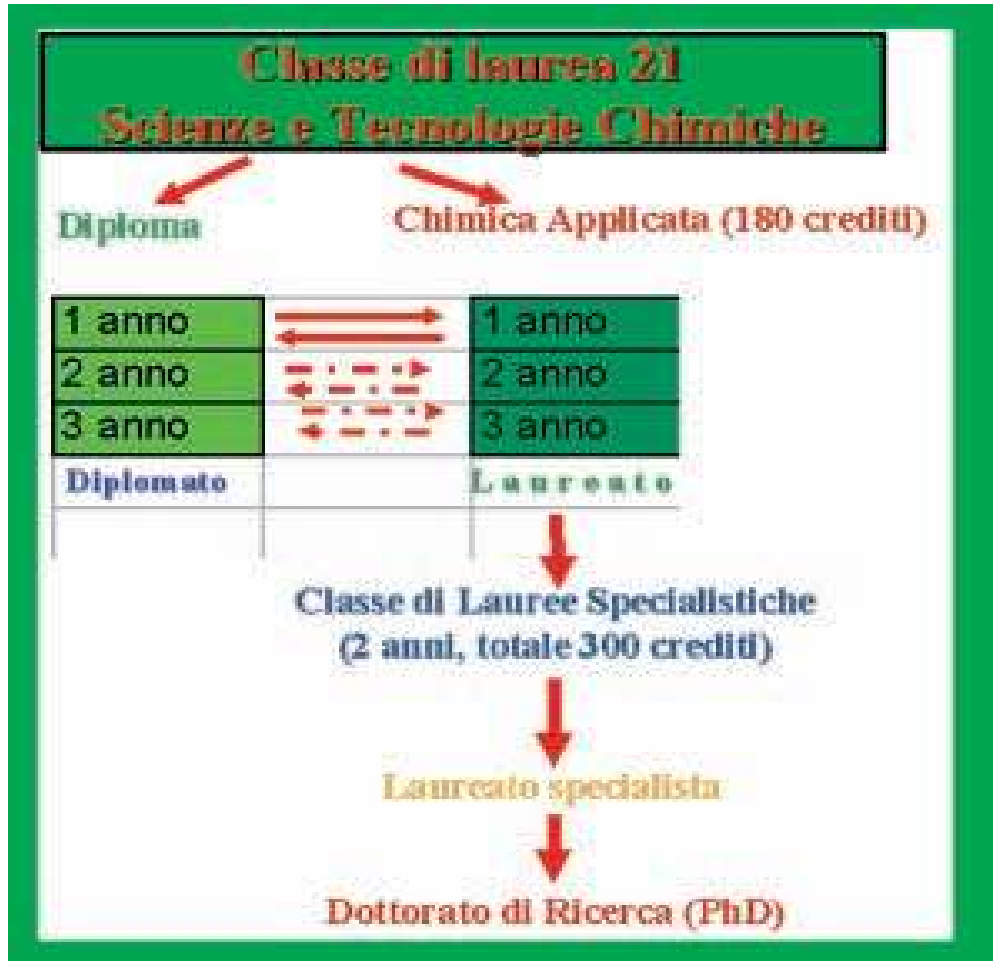

Figura 3 - Corrispondenza tra il Corso di Diploma in Chimica e la laurea triennale in Chimica Applicata ed il percorso di studi che è possibile seguire dopo la laurea triennale.

Allo scopo di soddisfare le richieste di formazione universitaria da parte di istituzioni dell'area fiorentina, il Corso di laurea in Chimica Applicata è stato strutturato in due percorsi formativi (figura 4):

- Chimica dei Processi

- Chimica Tessile

All'interno del percorso di studi in Chimica dei Processi è possibile ottenere una formazione più specifica verso la Chimica Ambientale o verso il Controllo delle Produzioni Chimiche scegliendo un itinerario di studi orientato secondo le specifiche esigenze dello studente. Questi percorsi di studio sono indicati nella guida dello studente e nei moduli di presentazione dei piani di studio.

La ripartizione dei crediti, delle ore di didattica e delle ore di studio previste per i vari settori scientifico disciplinari che sono necessarie per conseguire la laurea triennale in Chimica Applicata sono riportati nella figura 5. Si può osservare che agli studenti sono impartiti corsi di base di fisica e di matematica e un numero considerevole di corsi di natura chimica. È inoltre impartito allo studente l'insegnamento di una lingua straniera (generalmente inglese) ed un corso di informatica. 


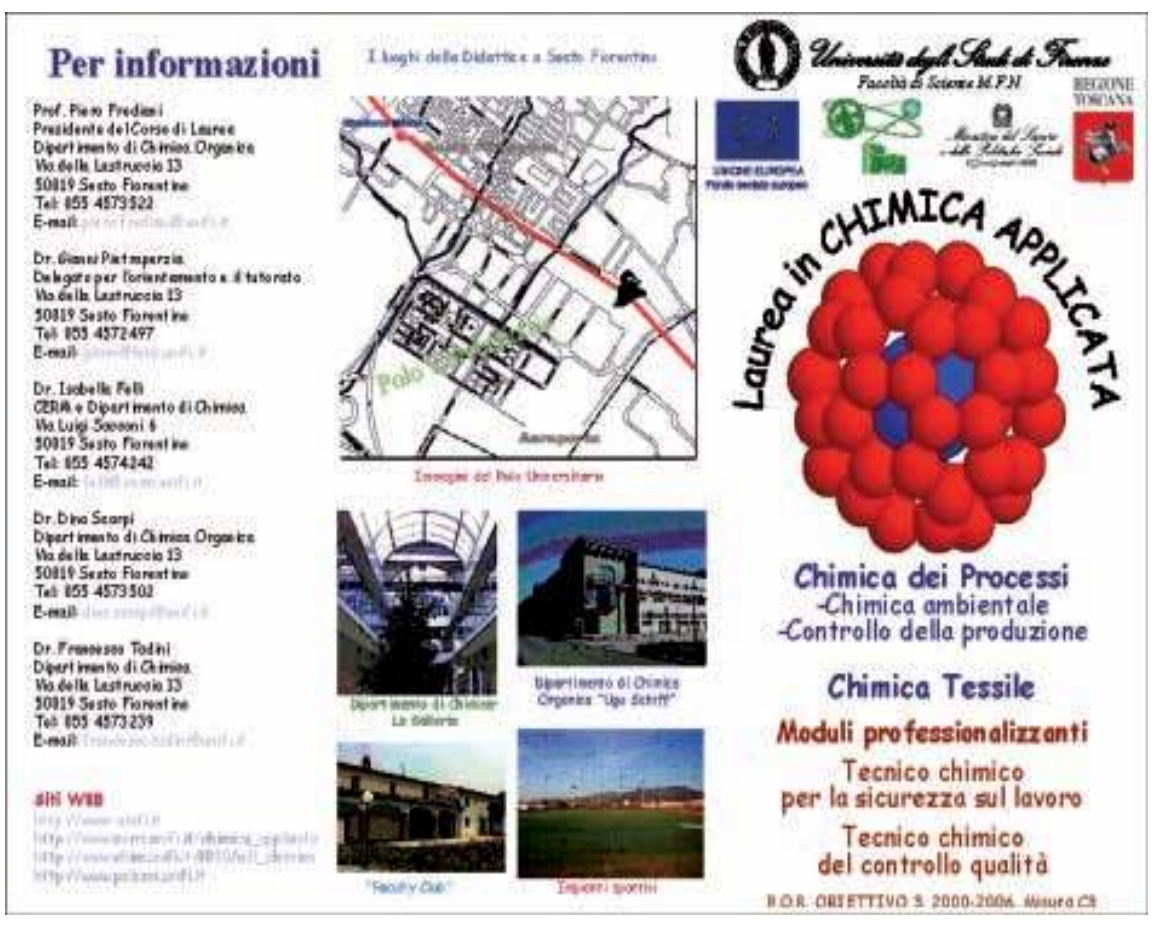

Figura 4a - Il manifesto del Corso di laurea in Chimica applicata.
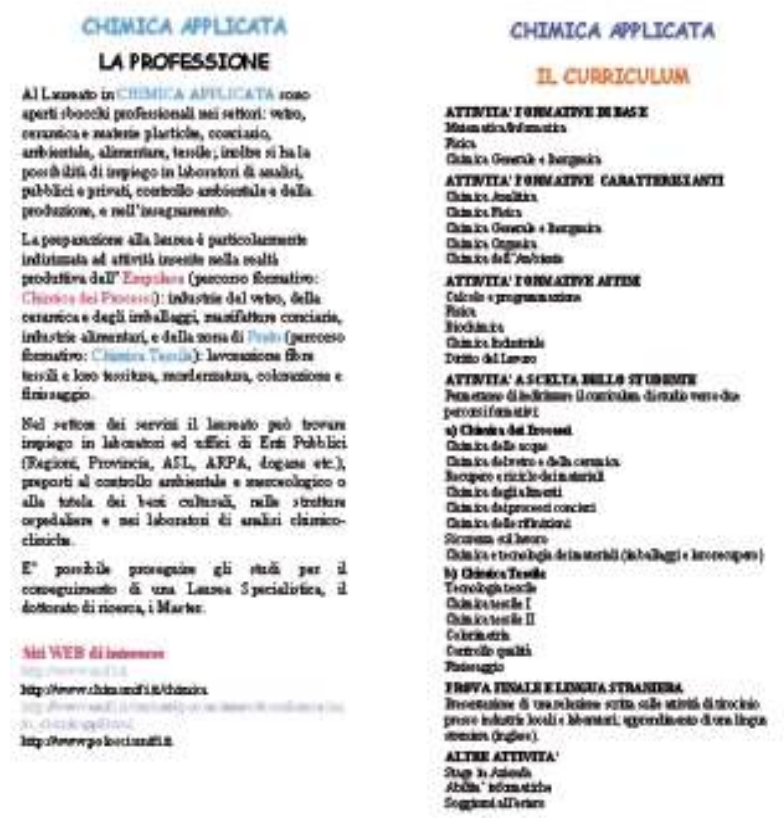

OHIMICA APPLICATA

LA DIDATTICA

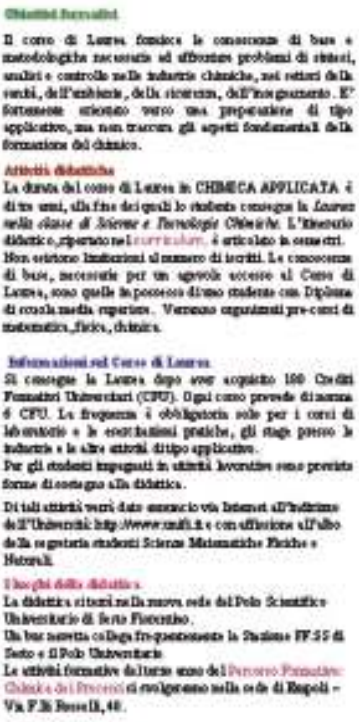

Figura $4 \mathrm{~b}$ - Il retro del manifesto del Corso di laurea in Chimica applicata. 


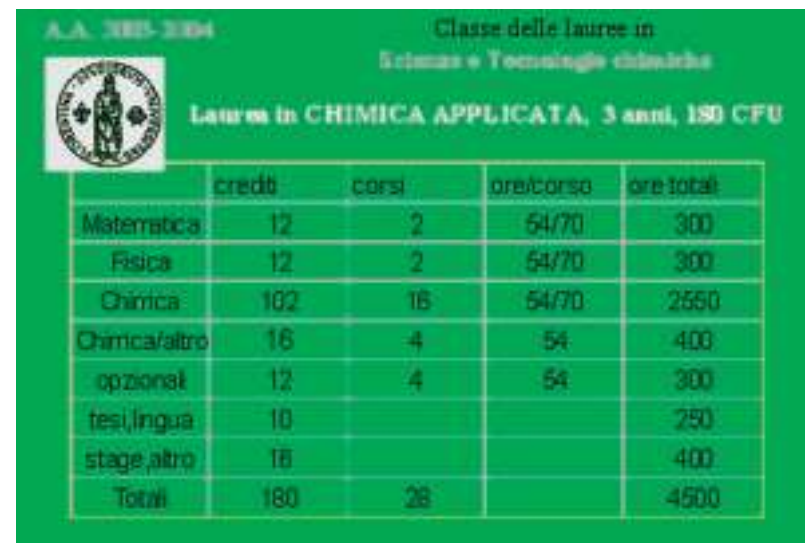

Figura 5 - Suddivisione dei crediti nel corso di laurea in Chimica Applicata.

Lo studente per completare il suo ciclo di studi e conseguire la laurea triennale, deve acquisire 180 crediti universitari come riportato nella figura 5. Il Corso di laurea in Chimica Applicata ha una eccellente offerta formativa come si evidenzia dal basso rapporto studenti/docenti, dalle strutture ed attrezzature messe a disposizione, fornisce agli studenti la possibilità di fare ricerca in Centri di eccellenza universitari o di fare uno stage presso un'azienda del settore chimico (figura 6). Al termine del percorso formativo uno studente consegue la laurea triennale in Chimica Applicata ed il titolo di dottore in Chimica Applicata.

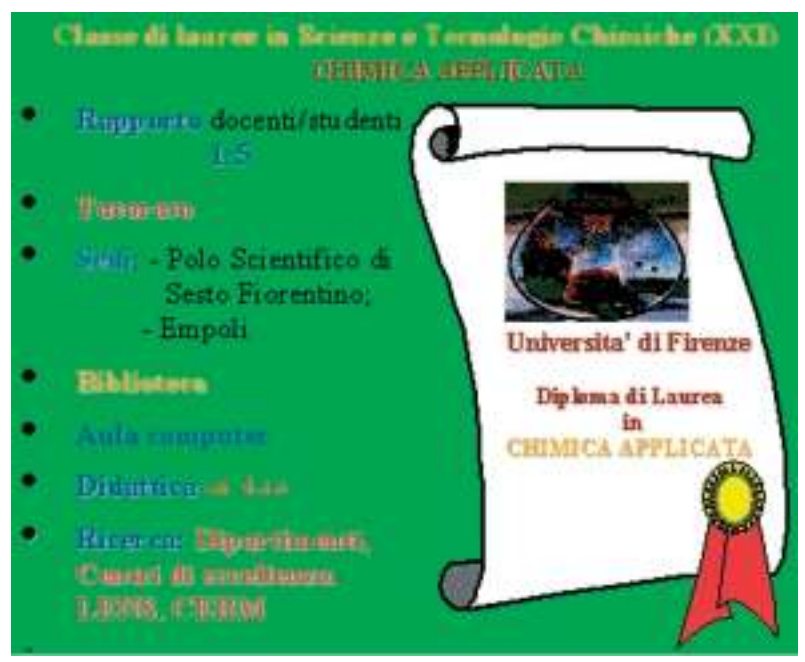

Figura 6 - Alcune caratteristiche del Corso di laurea in Chimica Applicata.

I corsi del III anno della laurea triennale in Chimica Applicata/orientamento processi sono stati mantenuti presso la sede universitaria di Empoli. Al fine di continuare a sostenere questa laurea, alla Associazione per il Diploma Universitario in Chimica di 
Empoli, che aveva completato il suo mandato essendosi concluso il corso di Diploma in Chimica, si è sostituito il Circondario Empolese Valdelsa (associazione tra i comuni dell'area empolese). È stata firmata una nuova Convenzione tra l'Università di Firenze e il Circondario Empolese Valdelsa in cui l'Università si impegna a mantenere ad Empoli il III anno del corso di laurea in Chimica Applicata ed il Circondario a sostenere tale offerta formativa fornendo le strutture didattiche necessarie ed un supporto finanziario.

Il corso di laurea in Chimica Applicata ha inoltre incrementato l'offerta formativa per i propri studenti al fine di un loro migliore e più rapido inserimento nel mondo del lavoro. A tale scopo nel percorso di studi universitario sono stati inseriti due moduli professionalizzanti. Tale inserimento è stato reso possibile in quanto il Corso di laurea ha partecipato, ed è risultato vincitore, di un bando della Regione Toscana per un finanziamento da parte del Fondo Sociale Europeo. Questi moduli professionalizzanti, sono stati inseriti nel primo e nel terzo anno del corso di studi universitari (figura 7).

I due moduli professionalizzanti sono stati inseriti al primo ed al terzo anno del corso di laurea in Chimica Applicata. Sono facoltativi, nel senso che uno studente può scegliere se frequentarli o meno e non incidono sulla sua carriera universitaria. Questi moduli possono essere frequentati anche da studenti esterni al Corso di laurea in Chimica Applicata. I due moduli professionalizzanti sono:

- Tecnico chimico per la sicurezza del lavoro

- Tecnico chimico per il controllo della qualità

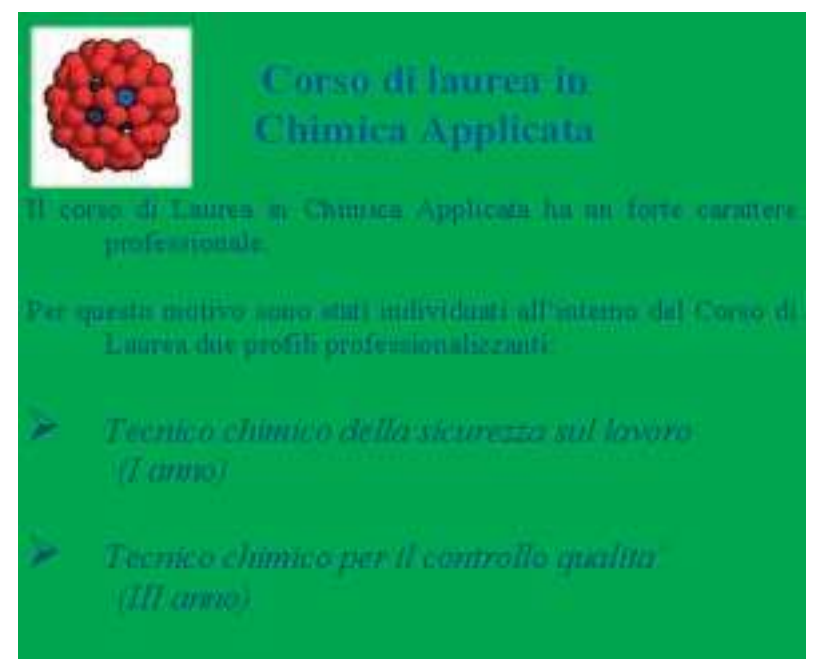

Figura 7 - I moduli professionalizzanti inseriti nel Corso di laurea in Chimica Applicata.

I moduli professionalizzanti si completano nel corso di un anno accademico, permettono un migliore collegamento tra l'Università ed il territorio in cui sono realizzati, formano delle figure altamente qualificate, facilitando un loro rapido inserimento nel 
mondo del lavoro ed infine rilasciano una qualifica che è riconosciuta dalla Regione Toscana (figura 8).

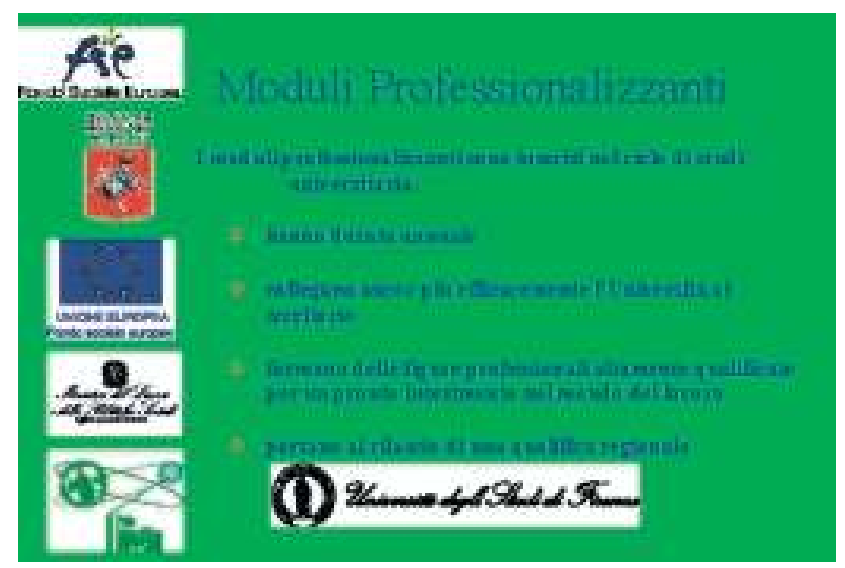

Figura 8 - Alcune caratteristiche generali dei moduli professionalizzanti.

Il modulo professionalizzante Tecnico chimico per la sicurezza del lavoro, che si tiene durante il primo anno di studi universitari del Corso di laurea in Chimica Applicata, insegna agli studenti quale sono le norme e le procedure che un lavoratore deve adottare per un corretto operare al fine della salvaguardia della salute e sicurezza proprie, delle persone che lavorano insieme a lui e dell'ambiente in cui opera. Questo risultato permette di controllare e minimizzare i rischi ambientali e salvaguardare la sicurezza delle persone che lavorano, delle strutture presenti e dell'ambiente interno ed esterno alla struttura operativa (figura 9).

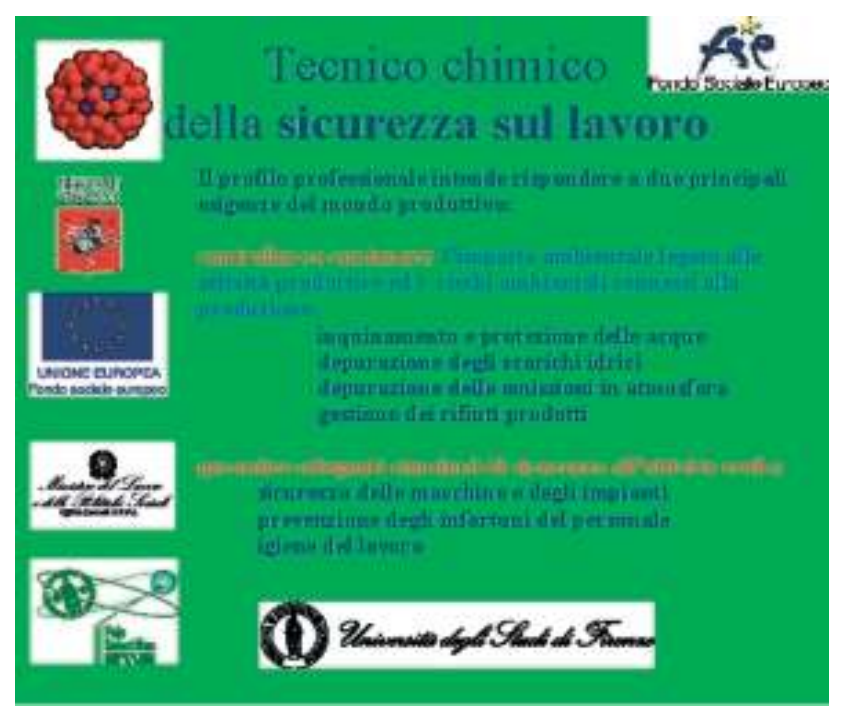

Figura 9 - Caratteristiche del modulo professionalizzante Tecnico chimico per la sicurezza del lavoro. 
I corsi che vengono impartiti e che sono ritenuti idonei a formare uno studente secondo i principi sopra esposti sono riportati nella figura 10. Allo studente, contemporaneamente alle prime nozioni chimiche, vengono impartite conoscenze di sicurezza del lavoro e di diritto del lavoro oltre ad una serie di seminari relativi alla cultura di impresa, alle tecniche di comunicazione, alla cultura europea ed alle pari opportunità. Durante il percorso di studi allo studente è richiesto di fare un tirocinio presso un laboratorio universitario oppure, se lo preferisce, uno stage presso una ditta esterna all'Università.

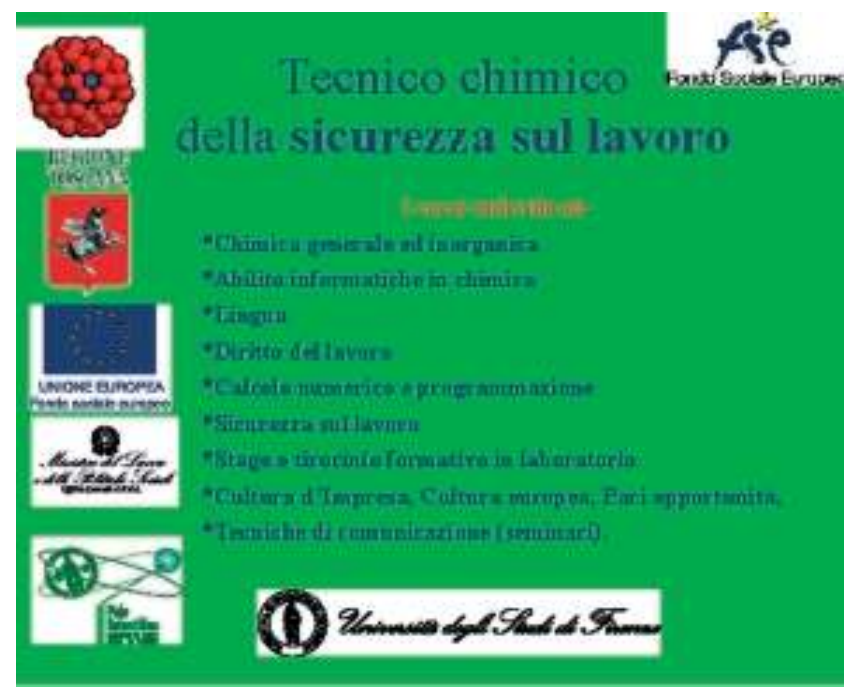

Figura 10 - Offerta formativa per il modulo professionalizzante Tecnico chimico per la sicurezza del lavoro.

Il modulo professionalizzante di Tecnico chimico per il controllo della qualità si tiene ad Empoli ed è dedicato agli studenti del III anno del corso di laurea in Chimica Applicata (figura 11). Tale modulo ha lo scopo di sensibilizzare gli studenti a lavorare secondo uno standard predefinito di qualità in accordo con quelle che sono le norme internazionali meglio conosciute come ISO 9000, ISO 9001 e, per l'ambiente ISO 14000, EMAS, ecc.

Questo modulo è stato inserito al terzo anno del corso di studi universitario perché necessita che gli studenti abbiano già acquisito le nozioni chimiche di base ed acquisiscano, contemporaneamente alle conoscenze chimiche professionalizzanti che permetteranno loro di inserirsi nel modo produttivo di una azienda e la sensibilità per lavorare secondo uno standard predefinito della qualità.

I corsi che gli studenti devono frequentare e superare variano secondo l'orientamento che lo studente ha scelto (figura 12). Si tratta comunque di corsi professionalizzanti che uniti ai corsi seguiti dagli studenti negli anni precedenti completano la loro formazione secondo i requisiti indicati.

Il percorso formativo del corso di laurea in Chimica Applicata si conclude con uno stage e la discussione di una tesi di laurea che ha per oggetto il lavoro fatto durante lo stage. 


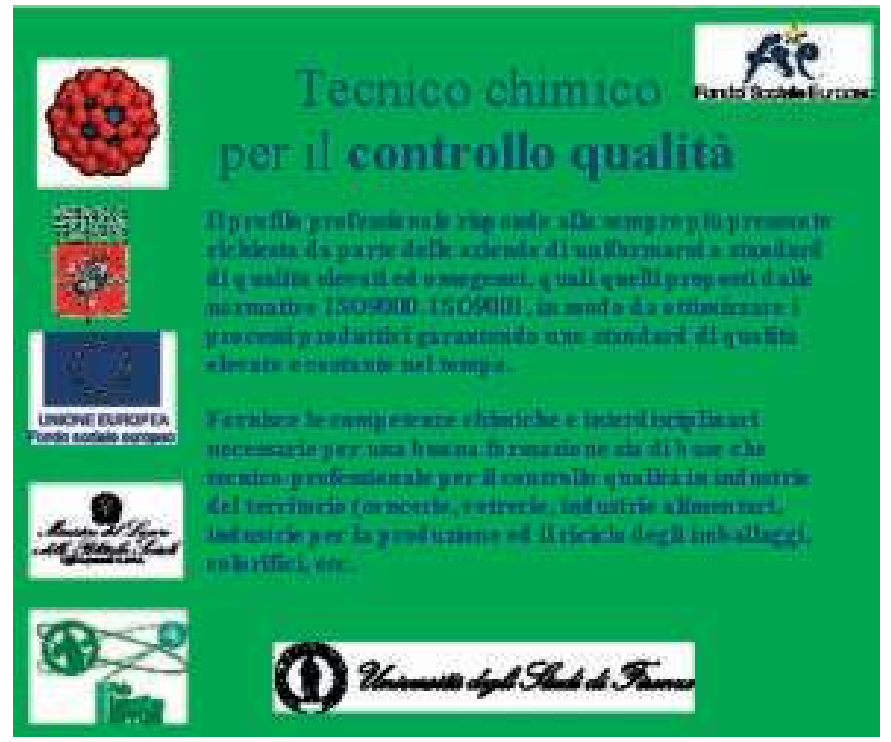

Figura 11 - Caratteristiche del modulo professionalizzante Tecnico chimico per la sicurezza del lavoro.

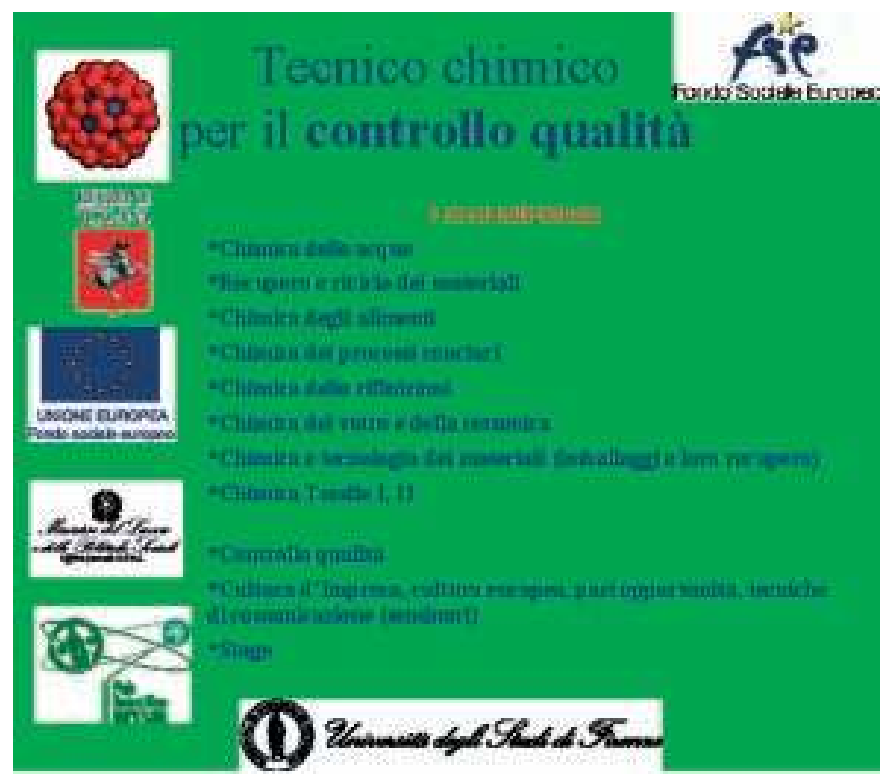

Figura 12 - Offerta formativa per il modulo professionalizzante Tecnico chimico per la sicurezza del lavoro.

Lo stage può essere effettuato sia presso i laboratori di ricerca universitari (figura 13) che presso una delle numerose aziende del settore chimico che si sono dichiarate disponibili ad accogliere uno studente del corso di laurea in Chimica Applicata e che il corso di laurea stesso in una visita preliminare ha ritenuto idonee perché in esse si possa effettuare lo stage (figura 14). 


\section{La ricerca CHIMICA a Firenze}

Ambiente

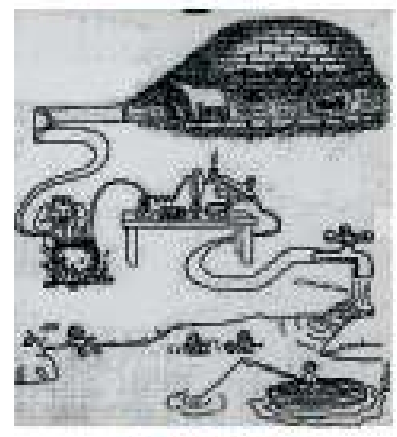

Chimica \&

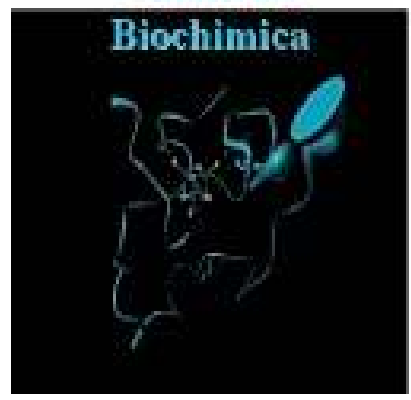

Scienza e restauro

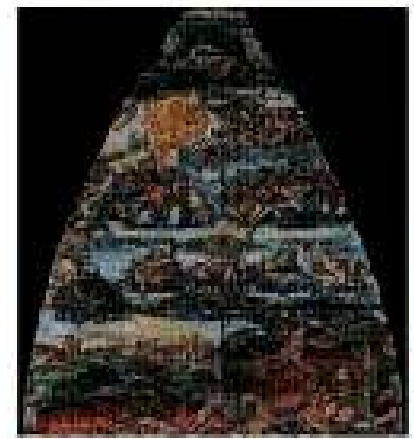

Mesochimica

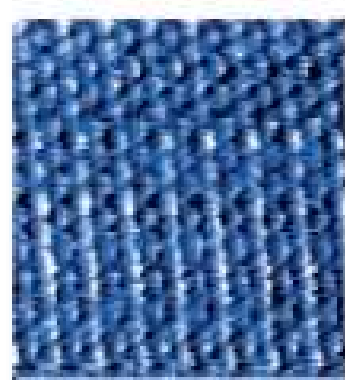

Nuovi materiali

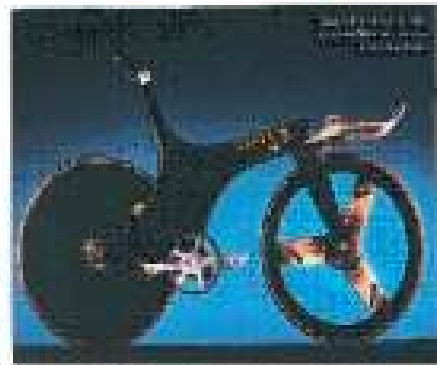

Fotochimica Laser

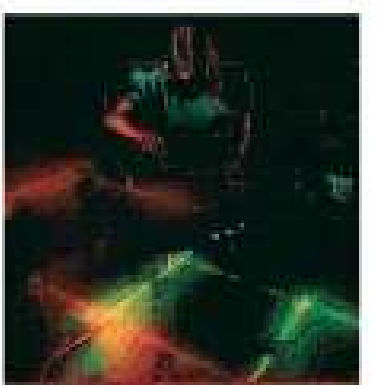

Figura 13 - La ricerca nei laboratori chimici dell'Università di Firenze.

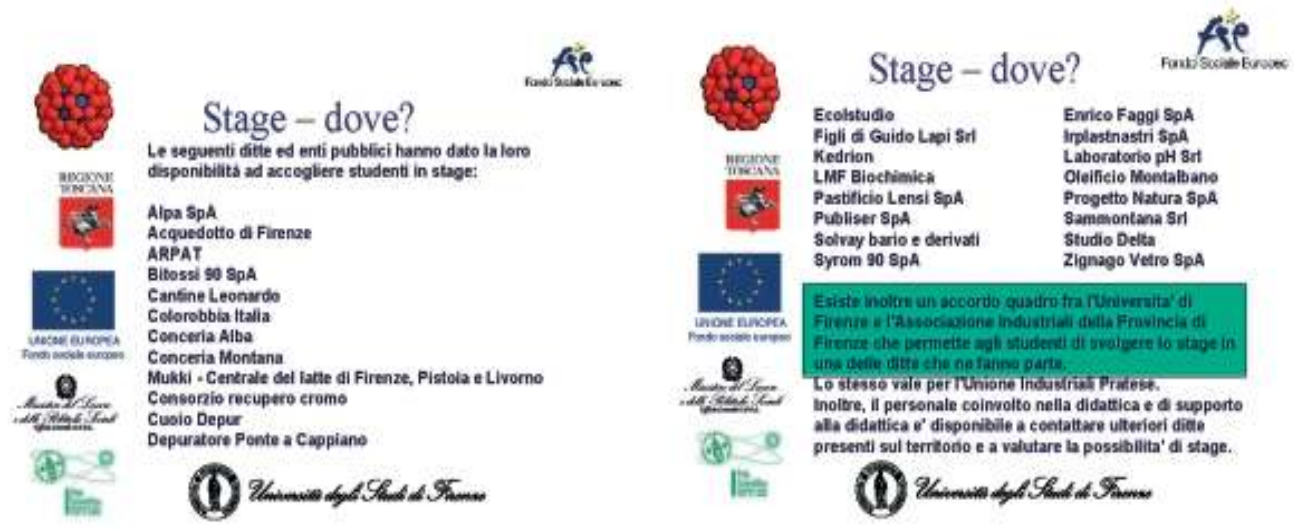

Figura 14 - Possibilità di stage aziendali presso il Corso di laurea in Chimica Applicata.

Ulteriori informazioni relative al Corso di laurea in Chimica Applicata possono essere ottenute utilizzando i riferimenti telematici riportati in figura 15.

Per concludere la maggior parte degli studenti del corso di laurea in Chimica Applicata trova un'opportunità di lavoro entro pochi mesi dalla conclusione del percorso di studi. 


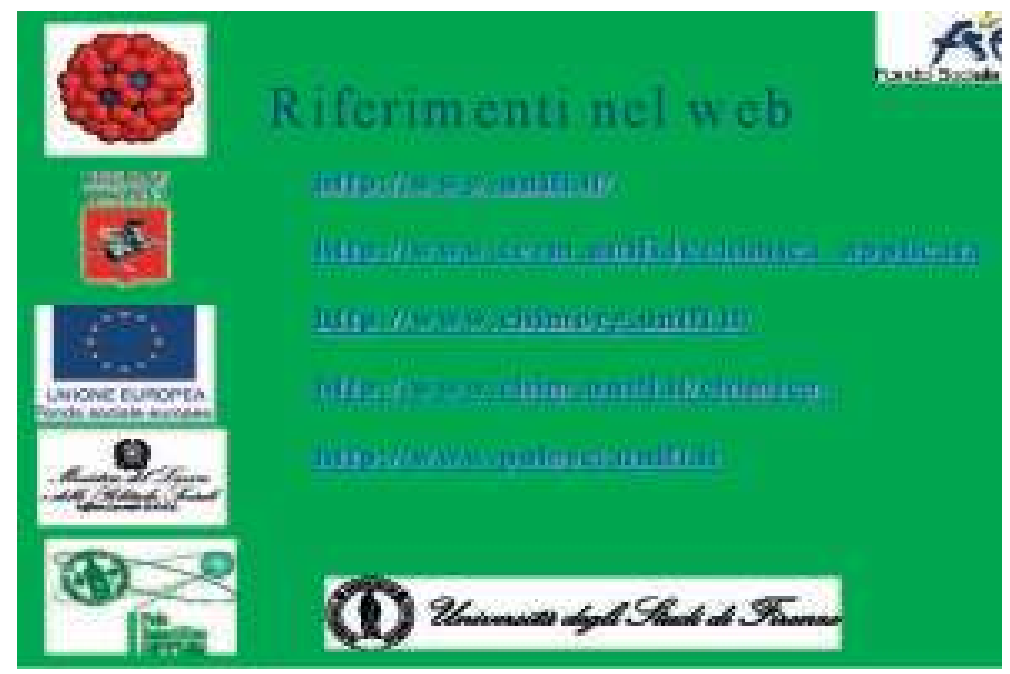

Figura 15 - Riferimenti telematici per il corso di laurea in Chimica Applicata. 
I

IL

7 


\title{
L'eredità di Leonardo: una ricchezza per l'ottica di oggi
}

\author{
di Silvano Abati, Emilio Borchi, ${ }^{* *}$ Alessandro Farini ${ }^{* * *}$
}

È inutile sottolineare ulteriormente la fama di Leonardo (figura 1) e l'importanza che le sue ricerche hanno avuto in moltissimi settori anche assai diversi tra loro. Importanza che può essere riscontrata in ogni angolo della cittadina (figura 2) che gli ha dato i natali, e che ospita l'Istituto Regionale Studi Ottici e Optometrici e il corso di laurea in Ottica dell'Università di Firenze. Potremmo dire con Romano Nanni che «non ci sono dubbi comunque che la riflessione sull'ottica accompagna tutta la vita di Leonardo, forse come nessun altro tema nella sua pur vasta e poliedrica attività: certamente perché il tema del vedere era fisiologicamente costitutivo della sua originale identità di artista-scienziato» [Nanni, 1998]. È infatti possibile riscontrare nelle sue opere moltissime osservazioni legate all'ottica. Allo stesso tempo, e non è l'unico paradosso di fronte a cui ci troviamo affrontando l'opera del grande vinciano, «dobbiamo dire subito che l'ottica non è il campo in cui Leonardo ha raccolto i frutti migliori, perché molto di ciò che ci ha lasciato a questo proposito perde quasi tutta la sua importanza attribuitagli, quando lo si inquadri nel complesso delle conoscenze ottiche del tempo» [Ronchi, 1968]. Leonardo infatti più che alla correttezza formale delle teorie sembra essere interessato a qualcosa che possa essere utile per la sua attività. Un esempio tipico lo abbiamo quando si vada ad osservare la sua posizione rispetto alla teoria della visione. Si combattevano all'epoca due teorie, quella estrusiva secondo cui vi erano 'raggi visuali', cioè bastoni infinitamente sottili, rettilinei, emessi dagli occhi e capaci di esplorare il mondo antistante all'osservatore, in modo da portare agli occhi gli elementi necessari alla visione, e quella intrusiva secondo la quale da ogni corpo, come descrive sempre Vasco Ronchi, partono in ogni momento come delle 'scorze' sottilissime, impalpabili, identiche per forma e colori al corpo emittente. La teoria dei raggi visuali aveva avuto nell'antichità enorme importanza, soprattutto perché veniva esposta nell' Ottica e nella Catottrica di Euclide. Si deve ad Alhazen (965-1040) una rivincita della teoria intrusiva.

* Direttore dell'Istituto Regionale di Studi Ottici e Optometrici.

${ }^{* *}$ Professore Ordinario di Fisica sperimentale, Facoltà di Ingegneria.

*** CNR Istituto Nazionale Ottica Applicata, Istituto Regionale Studi Ottici e Optometrici. 
Egli evidenzia molto chiaramente una serie di problemi che sorgono dall'ammettere che sia l'occhio a emettere qualcosa (solo per fare un esempio è difficile spiegare in questo modo le post-immagini che permangono sulla nostra retina quando chiudiamo gli occhi dopo aver osservato un oggetto luminoso). L'unico limite che rimaneva ad Alhazen era quello di ammettere che l'immagine potesse giungere sulla retina al contrario e ciononostante essere percepita diritta: per evitare di accettare questo apparente paradosso arrivò ad ipotizzare che fosse il cristallino a 'registrare' l'immagine.

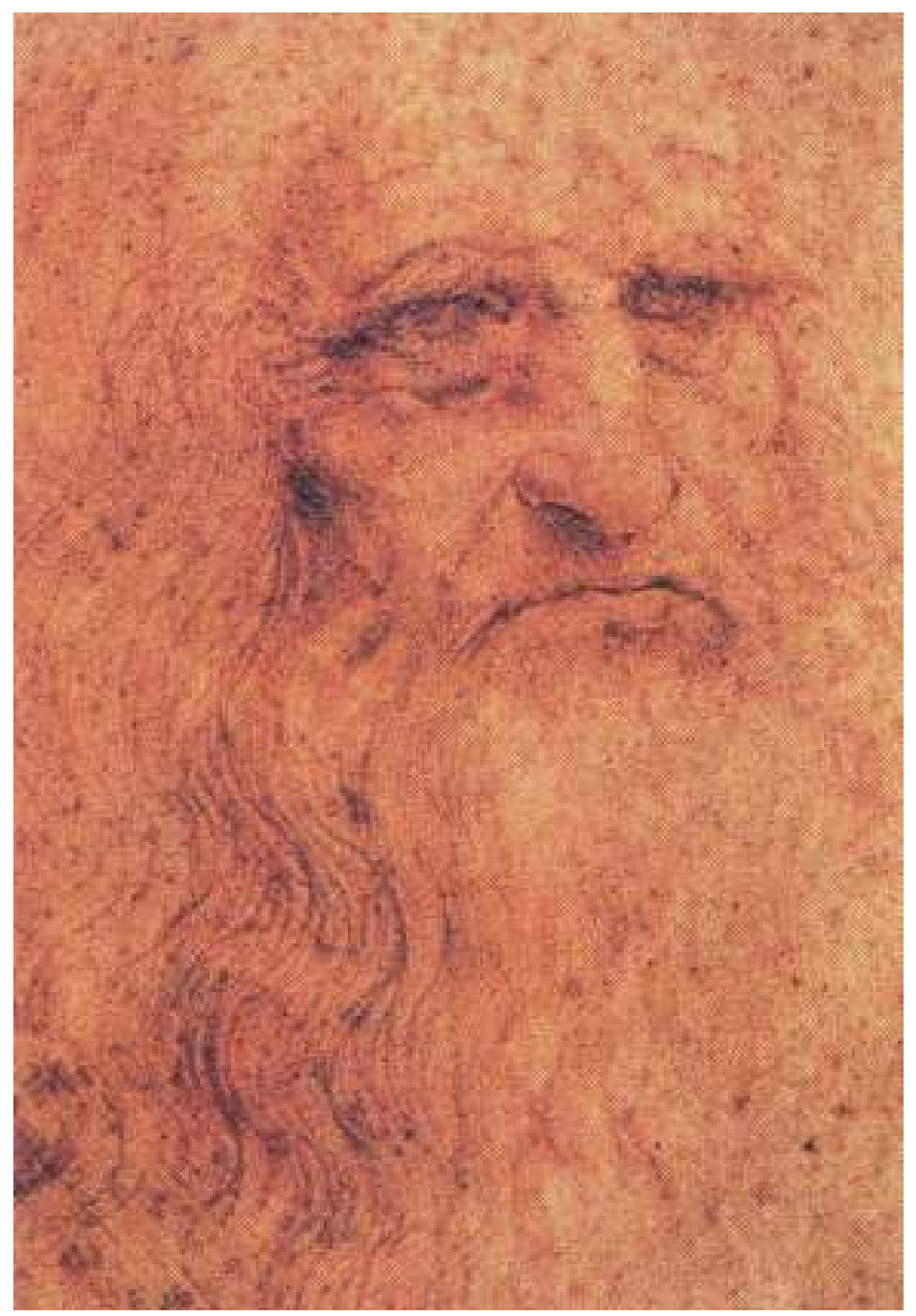

Figura 1 - Autoritratto di Leonardo.

Curiosamente Leonardo [Nanni, 1998] prende nel 1491 posizione per la teoria estrusiva: «Dico la virtù visivale astendersi per li razzi visuali in sino alla superfizie de' corpi non trasparenti e la virtù d'essi corpi astendersi in sino alla virtù visivale» (Codice 
atlantico fol. 729 v) e nel 1492 per la teoria intrusiva: «Impossibile è che l'occhio mandi fori di sé per li razzi visuali la virtù visiva...». Come spiegare questa contraddizione? La teoria estrusiva è in effetti assai poco realistica e sostenibile, ma ha un grande pregio: i raggi visuali permettono la costruzione di figure prospettiche e di ombre in maniera molto semplice. Era cioè una teoria sicuramente sbagliata, ma che forniva risultati utili nella pratica quotidiana e quindi poteva essere sostenuta da Leonardo. Anche per il genio di Vinci sorge poi il problema del ribaltamento dell'immagine che affronta da molti punti di vista, non sempre completamente corretti; il suo desiderio di capire questo problema lo spinge a ipotizzare la costruzione di un modello di occhio per ricreare il percorso dei raggi.

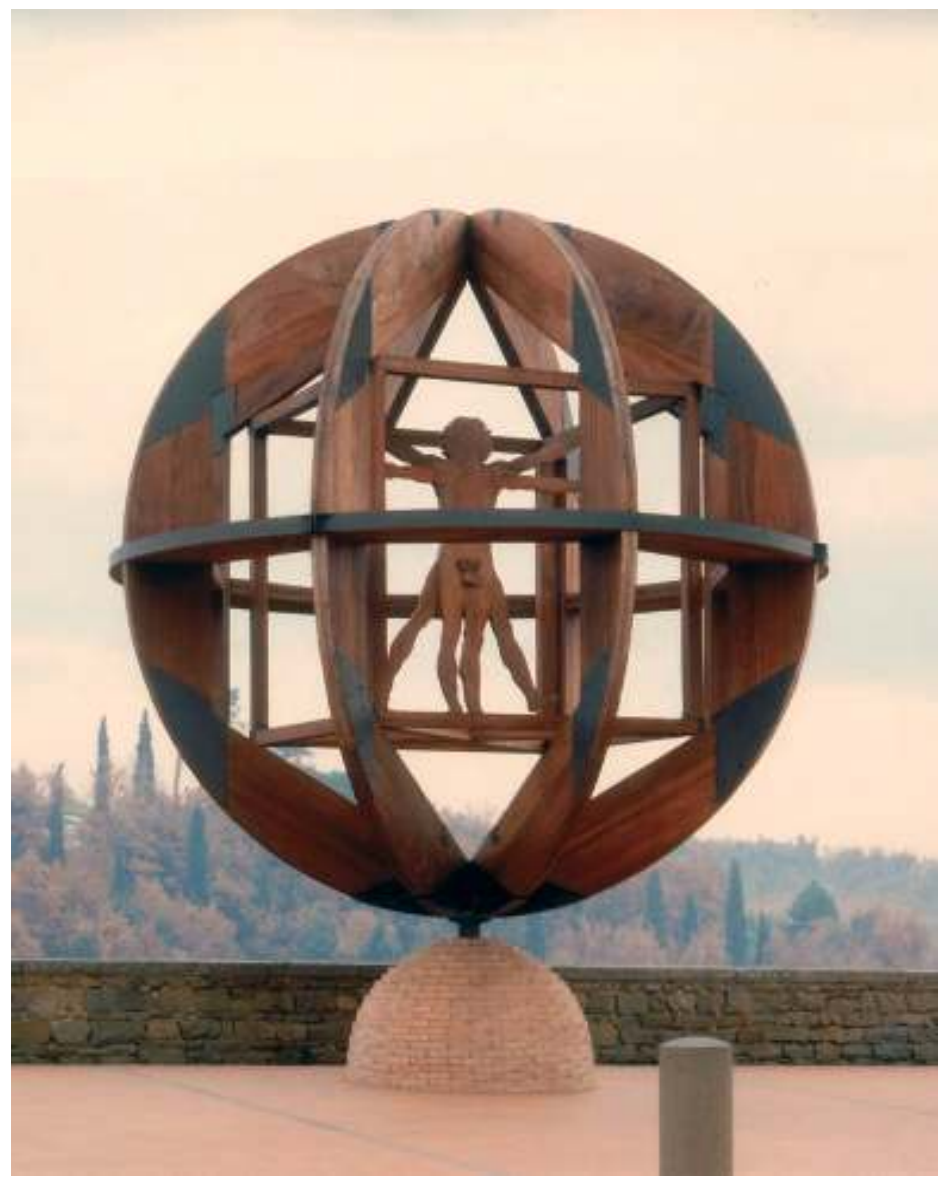

Figura 2 - L'uomo vitruviano in una piazza di Vinci.

Per comprendere un contributo decisivo dato da Leonardo nel settore dell'ottica è necessario capire la situazione dello studio delle lenti alla sua epoca. Gli occhiali erano già nati, probabilmente in ambito toscano verso la fine del 1200 , anche se la loro nascita non era stata accompagnata da una profonda comprensione delle leggi dell'ot- 
tica. Nei confronti delle lenti vi era però una profonda sfiducia da parte della scienza ufficiale. Le lenti non erano considerate uno strumento di conoscenza della realtà, poiché si inserivano tra soggetto e oggetto, generando illusioni; non ci si poteva fidare di ciò che si vedeva attraverso di esse. Le lenti erano nate fuori della scienza ufficiale («L'aver dato il nome di un legume a questi dischi di vetro è prova della loro origine artigiana: lenti di vetro, vetri a forma di lenticchia») [Ronchi, 1968], e vi rimarranno a lungo, al punto che anche il padre fondatore dell'istologia Xavier Bichat (1771-1802) fu molto restio nell'utilizzare microscopi. Leonardo invece valorizzò molto le lenti per tutte le possibilità che offrivano: più che interessato agli aspetti filosofici (è un'immagine reale o è illusione?) egli era interessato al fatto che funzionavano e potevano offrire grandi potenzialità. Così nel codice Atlantico possiamo trovare il famoso rigo «Fa ochiali da vedere la luna grande» (Cod. Atlantico fol. 190 a) oltre alla descrizione di macchine per la lavorazione di lenti e specchi. Il passo dedicato alla «luna grande» ha talvolta fatto pensare che Leonardo avesse costruito un cannocchiale: possiamo dire, seguendo uno dei personaggi storici dell'astronomia fiorentina, che «si è occupato del problema di vedere nitidi e ingranditi i corpi lontani e senza arrivare alla combinazione delle due lenti come Galileo, o al telescopio newtoniano deve aver fatto esperimenti che si avvicinavano molto all'invenzione di Newton" [Abetti, 1953]. Resta il fatto che sicuramente dedicò parte del suo tempo alla realizzazione tecnologica della componentistica ottica. Il foglio 1103 v. del Codice Atlantico, disegnato da Leonardo nel 1513, è dedicato ad esempio a macchine per la lavorazione degli specchi.

Di estremo interesse per coloro che studiano le lenti oftalmiche è un altro famoso passo: «Li ochiali quanto più li alontani dall'ochio [tanto] più mostrano magiori, quando sono di 50 anni. E se l'ochio vedrà due equali cose, al paragone l'una fori dell'ochiale e l'altra dentro, quella dell'ochiale li parà grande e l'altra picola. Ma le vedute cose deono essere lontane dall'ochio 200 braccia» (Cod. A fol. 12 v). Seguiamone con Vasco Ronchi [1968] la spiegazione; in primo luogo è interessante osservare che la frase «quando sono di 50 anni» indica che stiamo parlando di una lente positiva di circa 2 diottrie. Infatti all'epoca si usava indicare la potenza delle lenti specificando l'età di un presbite normale che necessitava di un tale potere. Accettando questa osservazione ecco che è corretto notare che se allontaniamo questa lente dall'occhio otteniamo un ingrandimento sempre maggiore (almeno per un certo intervallo). Allo stesso modo è giusto notare che se guardiamo un oggetto attraverso tale lente e ad occhio nudo, l'immagine ottenuta con la lente sarà più grande. A Vasco Ronchi, e noi con lui, non torna invece la necessità di avere un oggetto a 200 braccia, al punto che suggerisce un'errata interpretazione della grafia: il passo sarebbe corretto leggendo « 2 o 3 braccia».

Un contributo allo studio dell'ottica fu dato da Leonardo anche attraverso gli studi di anatomia (figura 3) per indagare il funzionamento del sistema visivo.

Possiamo così leggere «Nella notomia dell'occhio, per ben vederlo dentro senza versare il suo umore, si debba mettere l'occhio intero in chiaro d'ovo e far bollire, e soda ch'ell'è, tagliare l'ovo e l'occhio a traverso acciochè la mezza parte di sotto non versi nulla» (Cod. K fol. 119 r). Altra osservazione fisiologica, questa volta effettuabile senza la necessità di operazioni come quelle prima descritte, è quella relativa al 
restringimento della pupilla in presenza di luce: «La popilla dell'occhio si muta in tante varie grandezze quante son le varietà delle chiarezze e oscurità delli obbietti che dinanti se li presentano» (Cod. D fol. 5 v).

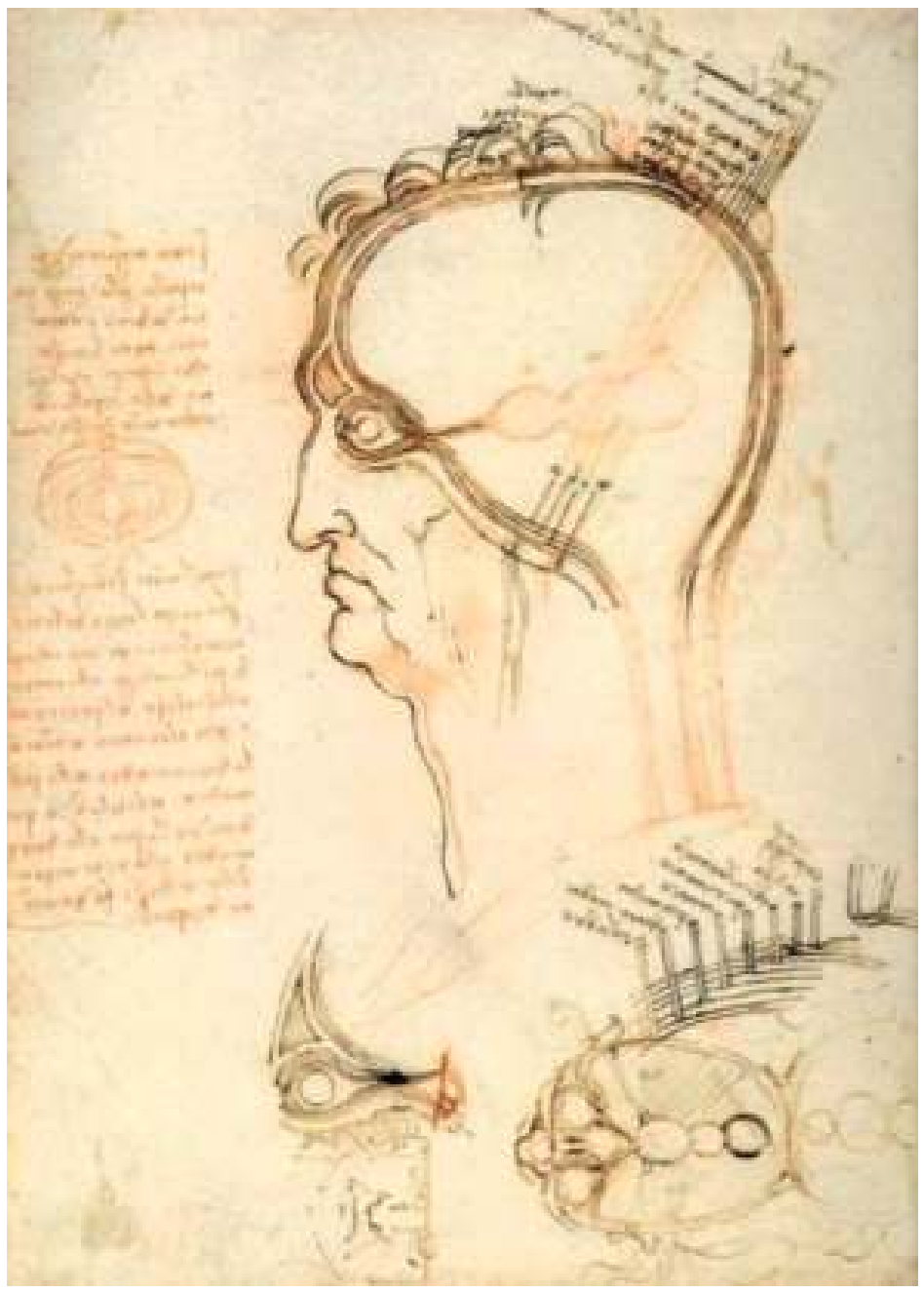

Figura 3 - Schema anatomico del sistema visivo.

Un capitolo vastissimo è ovviamente quello che potrebbe essere dedicato allo studio della camera oscura e della prospettiva, in cui non ci addentriamo, dato che potrebbero essere dedicati all'argomento volumi interi [Veltman]. Può però essere di interesse andare a rileggere la descrizione che Leonardo fa della camera oscura, senza entrare nel dibattito, oggi di gran moda, relativo al fatto che le opere dei grandi pittori del rinascimento siano state compiute con l'ausilio di strumenti ottici o con la sola abilità [Hockney, 2002]: «Pruova come tutte le cose poste 'n un sito sono tutte per tutto e tutte nella parte. Dico che, se una faccia d'uno edifizio o altra piazza o campagna che 
sia illuminata dal sole, arà al suo opposito un'abitazione, e in quella faccia che non vede il sole sia fatto un piccolo spiraculo retondo, che tutte le alluminate cose manderanno la loro similitudine per detto spiraculo e appariranno dentro all'abitazione nella contraria faccia, la quale vol essere bianca, e saranno lì appunto e sottosopra, e se per molti lochi di detta faccia facessi simili busi, simile effetto sarebbe in ciascuno» (figura 4).

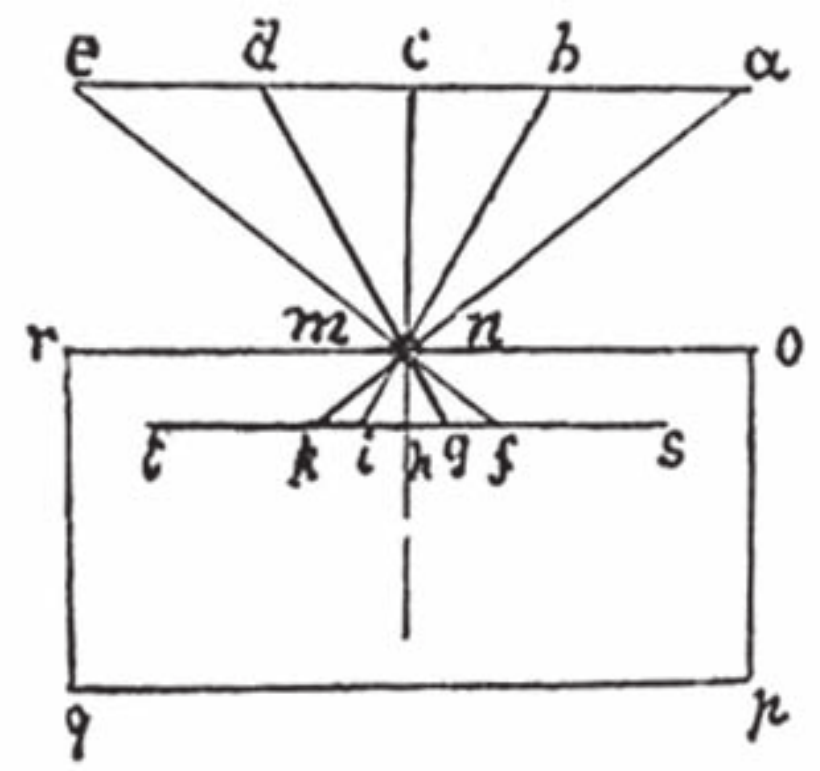

Figura 4 - Schema della camera oscura.

Ma per Leonardo lo studio della prospettiva non si limitava alle leggi matematiche del trattamento dei raggi; egli si rese presto conto che volendo rappresentare un paesaggio in lontananza era molto utile ricorrere alla cosiddetta 'prospettiva aerea' [Maffei, Fiorentini, 1995]. Si poteva cioè rappresentare i contorni sfumati e far assumere al paesaggio una dominante azzurrina, tenendo in questo modo conto della diffusione atmosferica. Questo è molto evidente in alcuni dipinti come ad esempio Sant'Anna, la Madonna e Gesù Bambino con l'agnello (figura 5) e La Vergine delle Rocce (figura 6).

Lo studio della prospettiva nei dipinti di Leonardo è sempre estremamente curato e attento, al punto che quando emergono imprecisioni, come quelle che un occhio allenato può cogliere ad esempio nell'Annunciazione, si può arrivare ad ipotizzare che non di errori si tratti, ma di una scelta voluta. Possiamo cioè dire con Antonio Natali di «mettere nel conto la possibilità che la tavola preveda un punto di vista privilegiato [...] quelle che paiono incongruenze potrebbero trasformarsi in espedienti compositivi, addirittura brillantemente escogitati» [Natali, 2000]. Saremmo cioè di fronte ad una specie di anamorfosi, un'immagine che acquista la giusta prospettiva se osservata ad esempio lateralmente. Uno studio che Leonardo aveva sicuramente presente dato che scrive: «E se dipingerai ciò su un muro davanti al quale potrai spostarti liberamente, ti sembrerebbe sproporzionato». 
Per un pittore come lui fu facile rendersi conto del fatto che il nostro occhio, più che valutare la luminosità (o per meglio dire la luminanza) assoluta degli stimoli, è uno strumento che funziona al meglio quando si trova a che fare con contrasti; in questo modo la luminosità di uno stimolo viene a dipendere criticamente dallo sfondo su cui si presenta (figura 7). Leonardo scriveva: «Quel riflesso sarà di più spedita evidenza, il quale è veduto in campo di maggiore oscurità, e quel fia meno sensibile, che si vedrà in campo più chiaro: e questo nasce che le cose di varia oscurità poste in contrasto, la meno oscura fa parere tenebrosa quella che è più oscura e le cose di varie bianchezze poste in contrasto, la più bianca fa parere l'altra meno bianca che non è».

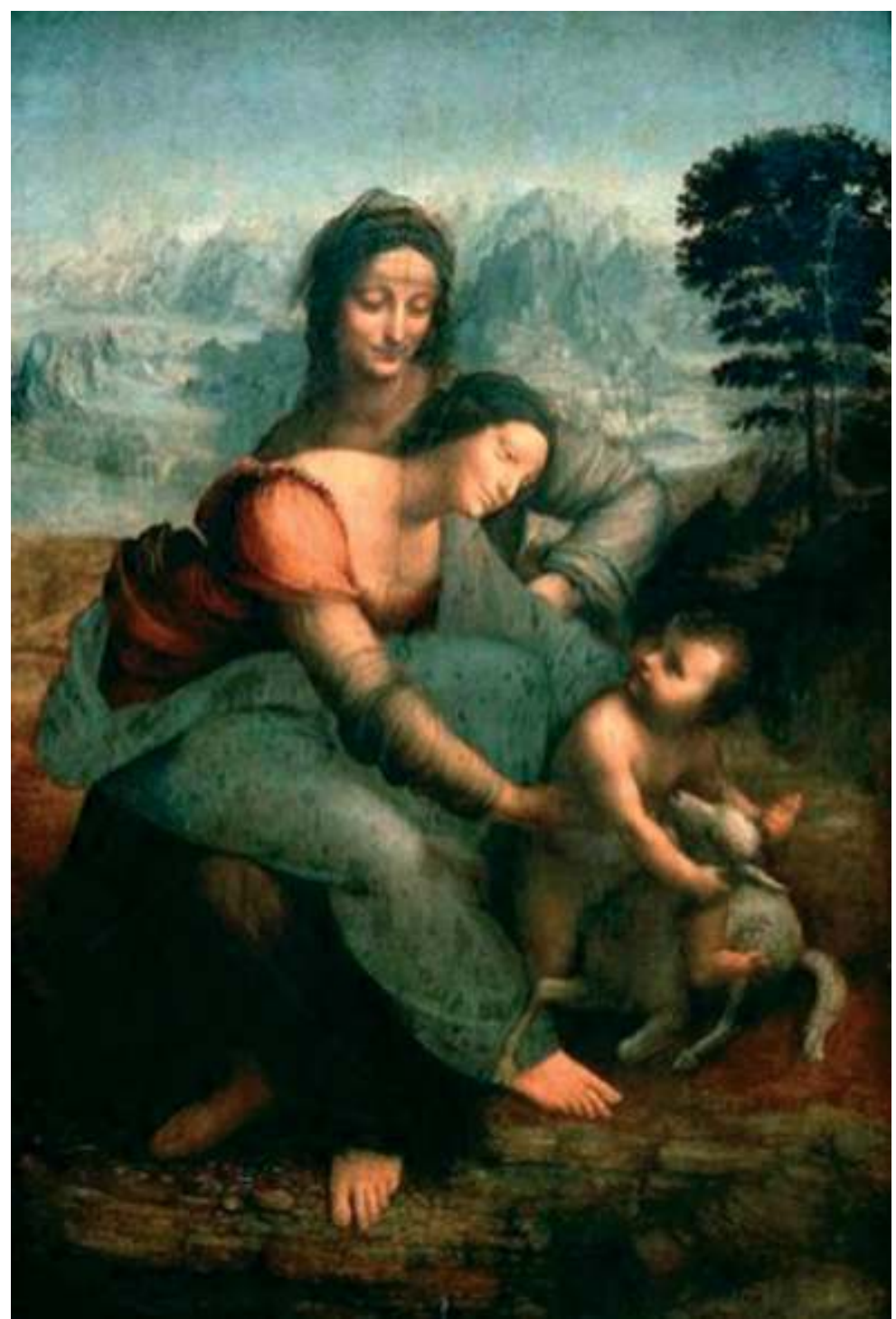

Figura 5 - Sant'Anna, la Madonna e Gesù Bambino con l'agnello. 


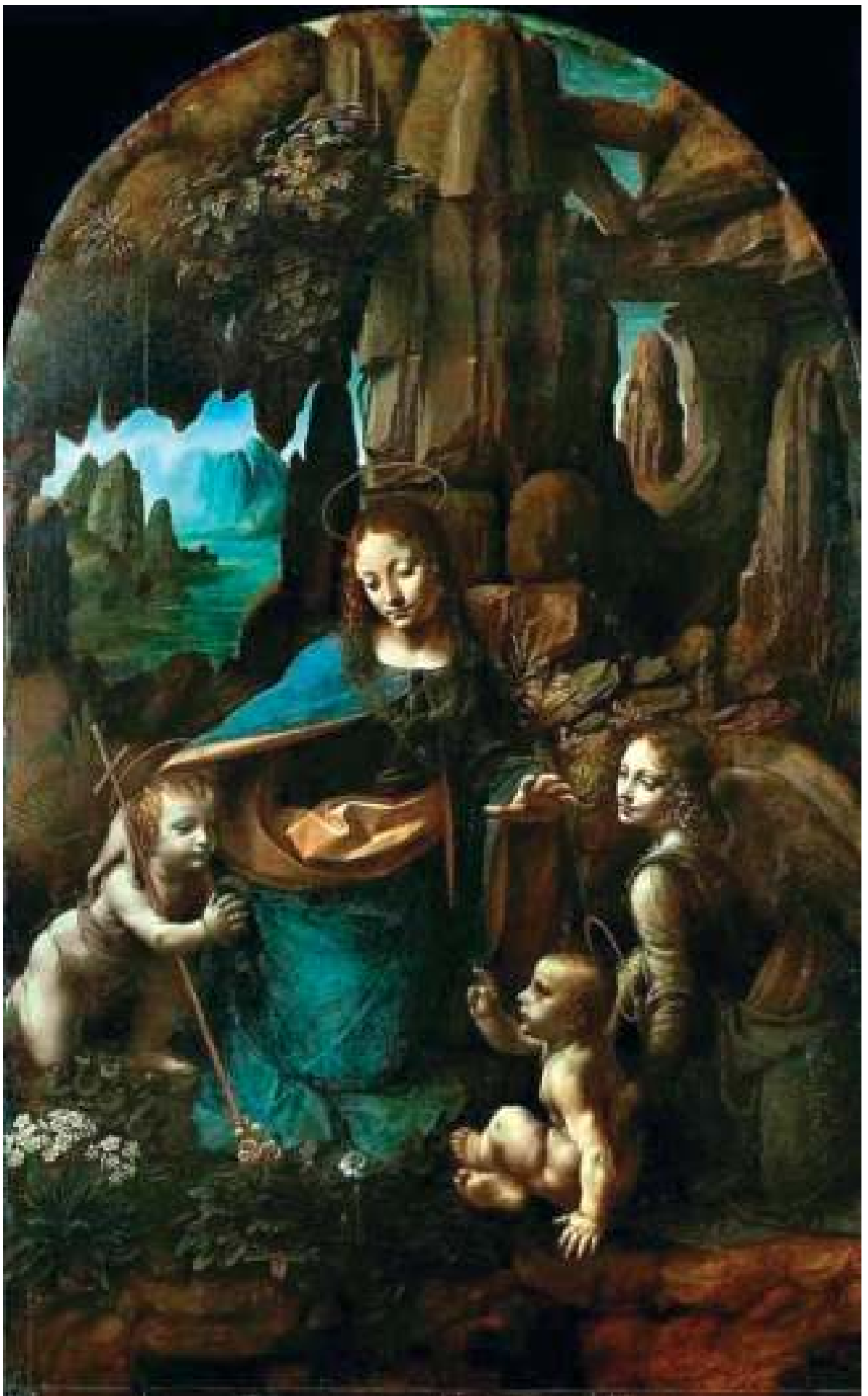

Figura 6 - La Vergine delle Rocce.

60 L'INNOVAZIONE PER LO SVILUPPO LOCALE Sivano Abati, Emilio Borchi, Alessandro Farini 


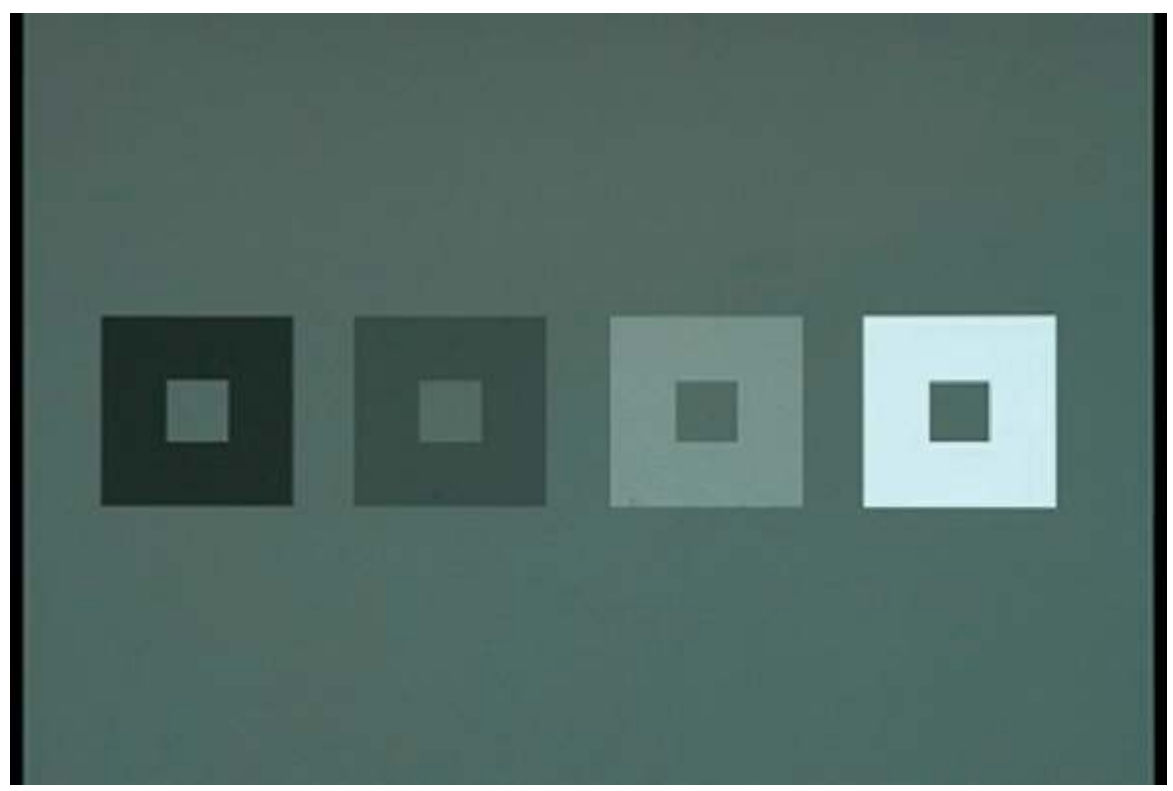

Figura 7 - I quadrati interni sono della stessa luminanza ma sono percepiti diversi a causa del diverso sfondo.

Per concludere, con un'operazione spericolata e forse discutibile, possiamo chiederci cosa penserebbe Leonardo del Polo Universitario Empolese, nato con un'attenzione grande alle necessità 'pratiche' della zona. Ci piace immaginare che sarebbe lieto di un'occasione di formazione nata nella sua patria, dato che nel Trattato della pittura (n. 77) scriveva: «Dell'errore di quelli che usano la pratica senza la scienza. Quelli che s'innamorano della pratica senza la scienza, sono come i nocchieri che entrano in naviglio senza timone e bussola, che mai hanno certezza dove si vadano. Sempre la pratica deve essere edificata sopra la buona teorica, della quale la prospettiva è guida e porta, e senza questa nulla si fa bene».

L'elenco dei meriti di Leonardo potrebbe continuare a lungo; possiamo però già in base a queste brevi note concludere che il da Vinci non fu solo un genio, ma uno sperimentale determinato e uno studioso attento a cogliere tutto ciò che nella realtà e in ciò che studiava metteva in moto la sua curiosità.

\section{BIBLIOGRAFIA}

Nanni, R. (1998), Leonardo e la teoria della visione, nel catalogo della mostra Scienza della Visione: aspetti e strumenti tra Leonardo e l'età moderna.

Ronchi, V. (1968), Leonardo e l'ottica, in Leonardo. Saggi e ricerche, 1954, e Scritti di ottica.

Abetti, G. (1953), Ottica e astronomia in Leonardo, estratto dagli Atti del Convegno di Studi Vinciani.

L'eredità di Leonardo

L'INNOVAZIONE PER LO SVILUPPO LOCALE $\mathbf{6} 1$ 
Veltman, K., Continuity and Discovery in Optics and Astronomy, <http://www.mmi.unimaas.nl/ people/Veltman/books/contin/title.html>.

Hockney, D. (2002), Il segreto svelato. Tecniche e capolavori dei maestri antichi, Electa Mondadori, Milano.

Maffei, L., Fiorentini, A. (1995), Arte e Cervello, Zanichelli, Bologna.

Natali, A. (2000), Dubbi, difficoltà e disguidi nell'annunciazione di Leonardo, in Natali, A. (a cura di), L’Annunciazione di Leonardo: la montagna sul mare, Silvana Editoriale, Milano. 


\title{
La formazione sanitaria ad Empoli da Vincenzio Chiarugi ad oggi
}

\author{
di Antonio Conti, ${ }^{*}$ Donatella Lippi**
}

Il 28 febbraio 1743, il Gonfaloniere e i Consoli di Empoli inviarono una supplica al Granduca, affinché "[...] gli assegnamenti lasciati dal Dottor Del Papa servino in l'erezione e mantenimento di uno spedale per gli infermi [...] perché in tutto il tratto fra Pisa e Firenze ed in Empoli, ancora non vi è alcuno spedale per i poveri infermi, i quali per lo più muoiono nelle proprie case senza assistenza [...]».

In effetti, la costruzione dell'ospedale San Giuseppe di Empoli si deve all'eredità del Dottor Giuseppe di Marco Del Papa.

Nato ad Empoli nel 1648, si era laureato in Medicina a Pisa, nel 1670; fu amico di Francesco Redi, rivestì cariche prestigiose, tra cui quella di membro del Collegio Medico Fiorentino, l'organismo deputato ad assegnare la Matricola, con cui si veniva autorizzati all'esercizio professionale.

Nel testamento, rogato in data 30 gennaio 1735 , egli lasciava i suoi averi per erogare borse di studio ai giovani empolesi, per un salario di un maestro di scuola e per aiutare giovani ragazze della sua città natale.

Dato che le somme elargite erano veramente cospicue, si arrivò alla decisione di utilizzarle per la costruzione dell'ospedale, intitolato a San Giuseppe, in memoria del Del Papa.

Veniva, in questo modo, colmata la lacuna di strutture sanitarie nel territorio.

Lospedale fu terminato nel 1749 e comprendeva due diverse infermerie e vari annessi.

Le Costituzioni per lo Spedale di San Giuseppe di Empoli furono approvate nel 1765, dopo che il Granduca Leopoldo era già diventato Imperatore.

L'Ospedale ha vita, come vedremo, ancora oggi, nonostante sia stato integrato dalla nuova struttura, edificata nel 1965 e raccoglie, senza soluzione di continuità, una tradizione che, anche secondo quanto viene affermato da Emanuele Repetti, affonda le sue origini in epoche molto antiche.

La storia dell'offerta sanitaria nel territorio empolese risale, infatti, al XII secolo, quando si registra la fondazione di strutture deputate all'assistenza, localizzate, però,

\footnotetext{
* Professore Associato di Fisica applicata, Presidente LM in Scienze delle professioni sanitarie della prevenzione, Facoltà di Medicina e Chirurgia, Università di Firenze.

${ }^{* *}$ Professore Associato di Storia della medicina, Facoltà di Medicina e Chirurgia.
} 

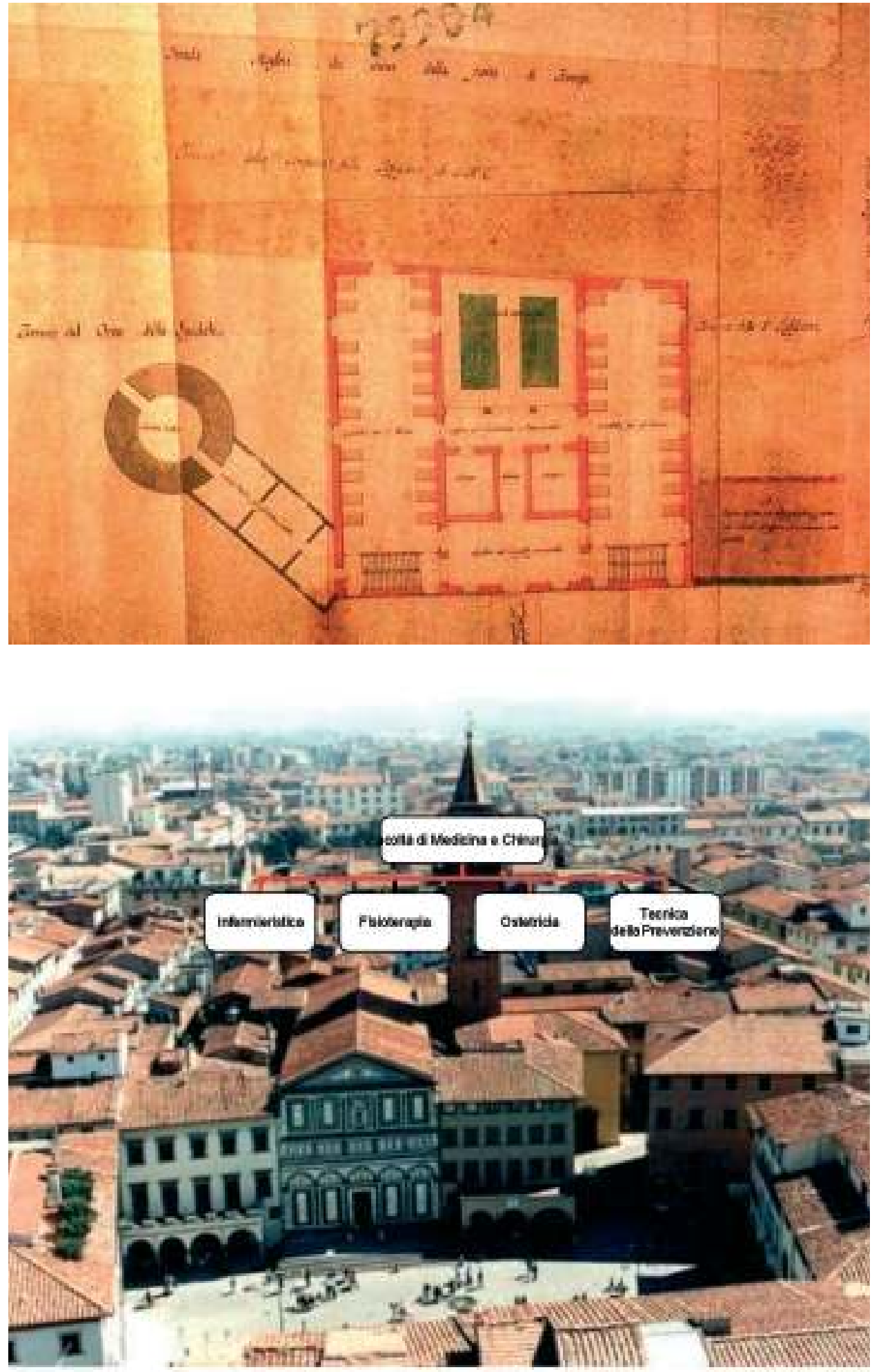

64 L'INNOVAZIONE PER LO SVILUPPO LOCALE

Antonio Conti, Donatella Lippi 

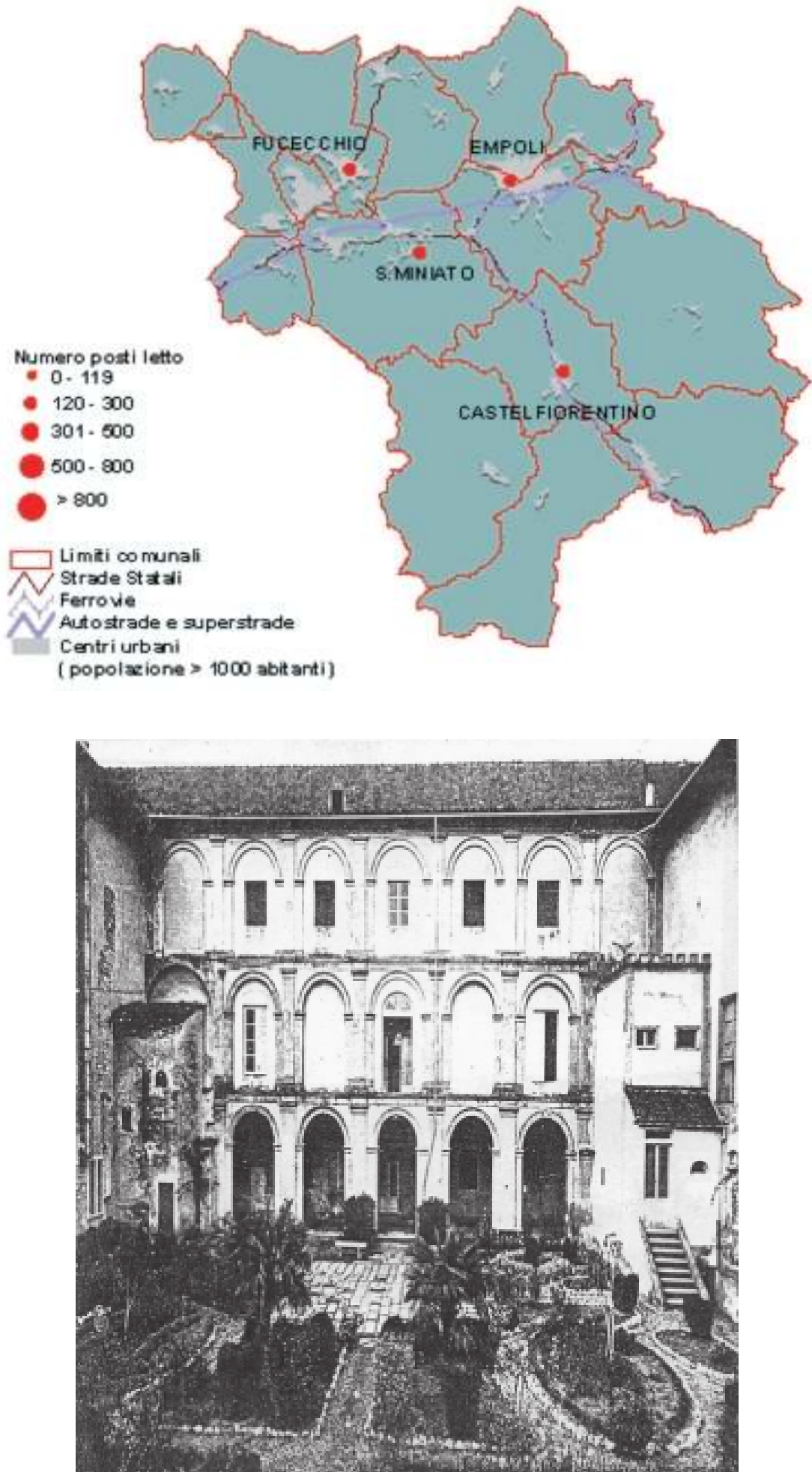

La formazione sanitaria ad Empoli

L'INNOVAZIONE PER LO SVILUPPO LOCALE 
nei centri maggiori, come l'Ospedale di San Salvatore, a Fucecchio, l'Ospizio di San Jacopo a Castelfiorentino, l'Ospedale di San Martino a San Miniato.

I toponimi rivelano retrospettivamente questa vocazione del territorio: basti pensare al nome di 'Spicchio', derivante, con ogni verosimiglianza, dal termine Hospiculum, come luogo di ricovero per i pellegrini.

Non erano, però, vere e proprie strutture a fini sanitari, ma genericamente assistenziali.

Risale al XIX secolo, infatti, la fondazione dell'Ospedale di San Pietro Igneo a Fucecchio, che fu realizzato verso gli anni trenta del 1800, dedicato a Pietro Aldobrandini che, nel 1068, uscì indenne da un rogo che aveva attraversato, come in una sorta di ordalia, per confermare la illegittimità della nomina del vescovo di Firenze, Pietro Mezzabarba.

Anche Castelfiorentino ebbe, a partire dal Basso Medioevo, diverse strutture di accoglienza, ma, nel corso dell'Ottocento, venne fondato l'Ospedale di Santa Verdiana, che, in questi ultimi anni, è diventato Ospedale generale di zona.

Proiettati sul pisano sono, invece, gli Ospedali Riuniti di San Miniato, che vennero riorganizzati in età leopoldina, ma che raccoglievano un'eredità molto più antica. Se, del resto, nel territorio empolese, mancò a lungo una vera e propria struttura sanitaria, la presenza di personale medico di spessore era invece ben attestata e investigare questa documentazione consente di ripercorrere retrospettivamente anche le vicende sanitarie del periodo.

Queste presenze sono particolarmente significative, grazie anche alla maggiore disponibilità di fonti, tra XVIII e XIX secolo.

Due personalità, per motivi diversi, appaiono di grande interesse, una per la Medicina, l'altra per la Chirurgia.

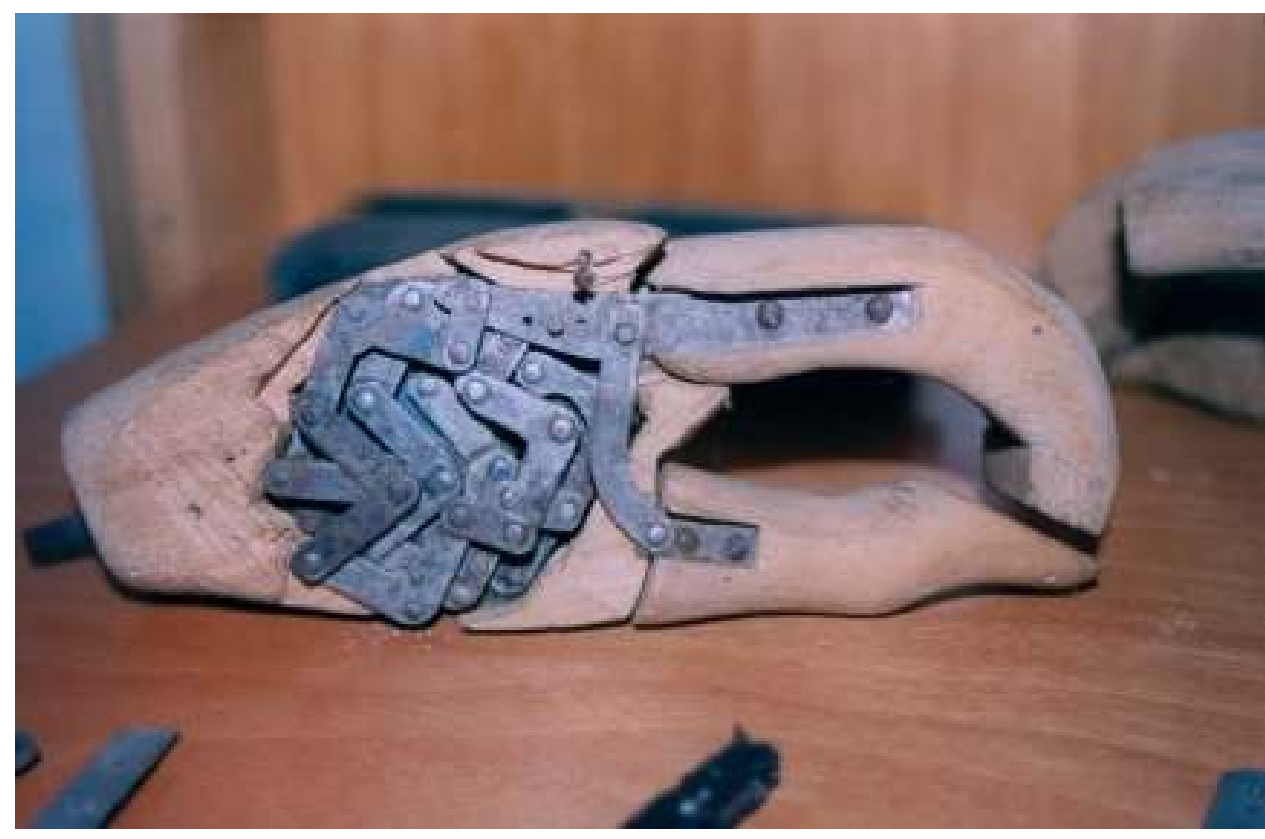

Protesi realizzata da Giuliano Vanghetti e conservato presso il nucleo museale della Biblioteca Comunale R. Fucini di Empoli (Firenze). 

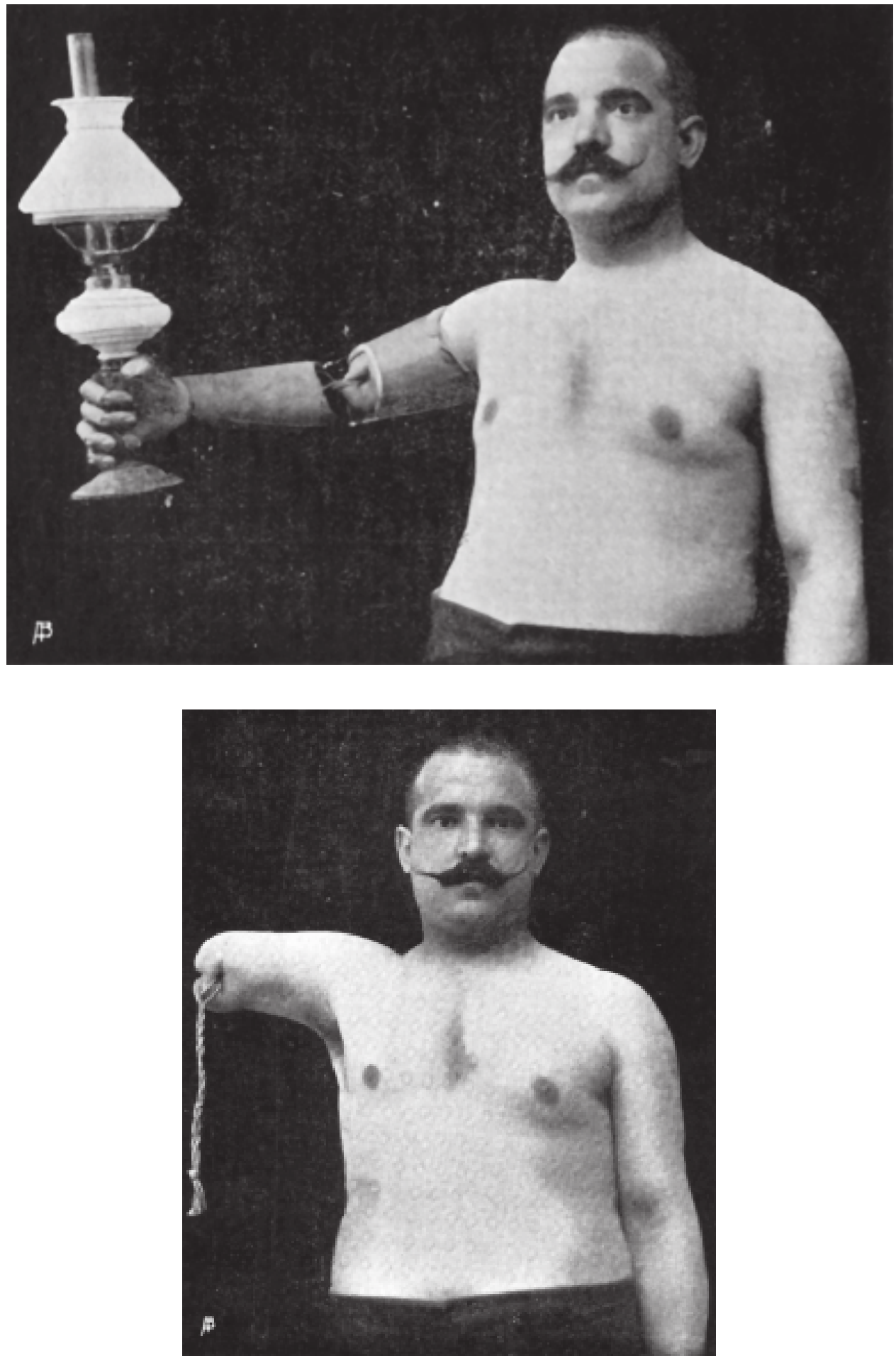

Recupero delle funzioni secondo il metodo di G. Vanghetti. 


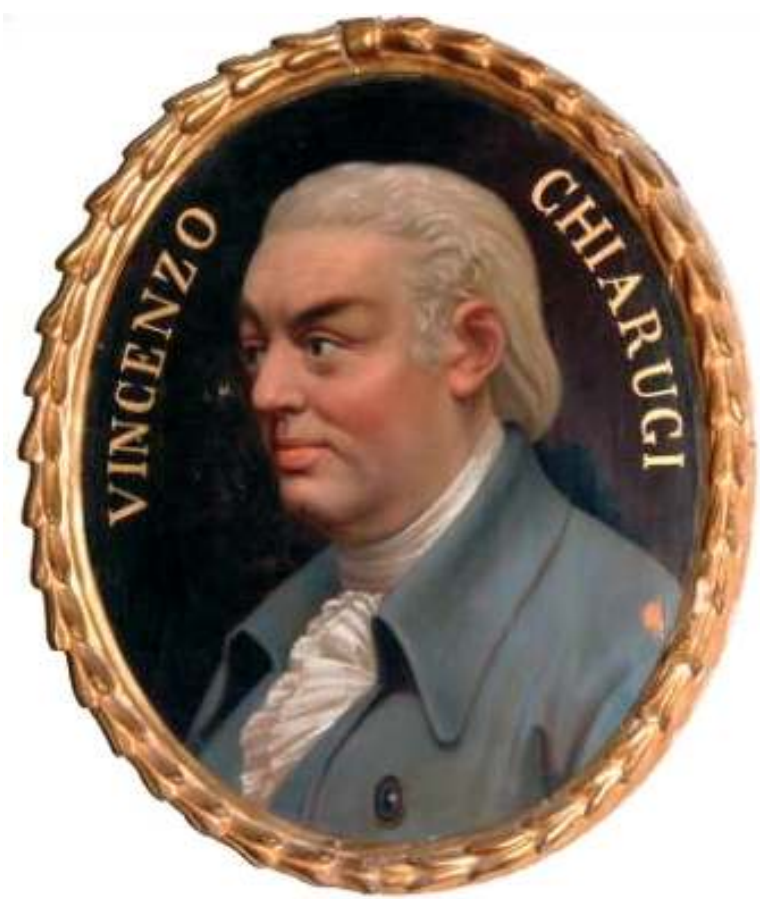

Vincenzio Chiarugi. Ritratto conservato presso la Biblioteca Biomedica di Careggi (Firenze).

Due destini opposti, se vogliamo, che sono accomunati, però, da un analogo atteggiamento nei confronti della persona che ha bisogno; un 'prendersi cura', che va al di là del dovere professionale e che trova, nei fondamenti scientifici, gli elementi per procedere a una rivisitazione del trattamento terapeutico.

"Ci vedremo a Empoli forse per la fiera ed allora ti porterò di prova un cannocchiale olandese ed abbracciandoti e portandoti a saggiare la mia inaugurale ...».

Così scrive Vincenzio Chiarugi, il 10 settembre 1802, all'amico e collega Cosimo Salvagnoli, personaggio di grande spicco nella Empoli di fine Settecento-inizi Ottocento.

Medico, provveditore del Monte Pio, Cosimo Salvagnoli è il destinatario del corpus di lettere, conservate nell'Archivio Storico del Comune di Empoli, scritte da Vincenzio Chiarugi tra il 1794 e il 1820.

«Portandoti a saggiare la mia inaugurale...»; si tratta della lezione inaugurale del Corso di Malattie Cutanee e Mentali che Chiarugi tenne dal 1802 come Lettore Onorario dell'Università di Pisa presso la Scuola di Complemento e Perfezionamento di Santa Maria Nuova di Firenze.

In effetti, presso la Scuola fiorentina, in quegli anni, si stavano verificando mutamenti di grande importanza, tra cui, appunto, l'istituzione di nuove cattedre di insegnamento.

Presso Santa Maria Nuova, infatti, venivano compiuti gli ultimi due anni del Corso di Laurea in Medicina e Chirurgia, iniziati presso le Facoltà di Pisa e di Siena.

La fondazione di nuovi insegnamenti risponde sempre a esigenze di carattere scientifico, ma, soprattutto, sociale: la necessità di creare una cattedra che si centrasse su un'emergenza 
dermatologica e psichiatrica, infatti, traduceva dal punto di vista istituzionale la diffusione di una patologia che assommava in sé questo apparente doppio carattere clinico.

Stiamo parlando della pellagra, il mal della rosa, che fino al 1914, quando Funk ne individuerà l'agente responsabile, rimarrà un mistero dal punto di vista eziologico, ma che era stata già compiutamente descritta nei secoli precedenti, nelle sue manifestazioni cutanee e nelle complicanze neurologiche.

Non a caso, nella Fabbrica di Bonifazio, in via San Gallo, dove oggi ha sede la Questura, vennero ricoverati, dal 1789, i Cutanei e i Dementi.

Vincenzio Chiarugi ebbe una carriera faticosa: solo dal 1805 il suo impegno universitario ebbe un riconoscimento economico e la conferma dell'incarico di Soprintendente alle Infermerie e agli Studi in Santa Maria Nuova gli giunse soltanto un anno prima della morte.

Nell'epistolario con Cosimo Salvagnoli, emergono le istanze più personali della sua biografia, tra cui il legame con la città natale, dove, nel febbraio del 1805 , vendette $\mathrm{i}$ suoi beni, dopo aver acquistato una villa sulla collina di Careggi, nella valle del Terzolle.

Molto probabilmente, nella valutazione del personaggio Chiarugi, figlio del sapere medico che caratterizzò il passaggio tra Settecento e Ottocento, ha influito negativamente la 'fuorviante' tesi del 'primato' rispetto a Pinel, che, nello stesso anno, liberava dalle catene i dementi rinchiusi nella Salpetrière.

Chiarugi si confronta a pieno campo con la medicina del suo tempo e lo fa, accogliendo le concezioni di Cullen e di Brown: la novità del suo apporto, al di là dell'approccio filantropico, sta proprio nella inedita messa a fuoco della funzione nervosa, sia in psichiatria, sia in medicina.

Il folle viene finalmente collocato nel contesto della medicina e ne viene fondata la curabilità, ma non è un'operazione semplice, in quanto Anima e Sensorio Comune non sono legati semplicemente da un rapporto spaziale e non trovano una loro articolazione nella complessa fisiologia di Chiarugi.

La matrice illuminista, che presiede l'opera di Chiarugi, è fortissima, ma effimera: sul piano del contributo culturale-scientifico, la sua riforma si esaurisce, ma, in seguito, in Toscana e nell'Italia post-unitaria si evidenziano i percorsi che possono essere ricondotti a Chiarugi stesso.

Il termine 'precursore', che tanto spesso è stato attribuito a Chiarugi, nell'ottica della moderna storia della medicina non trova più una sua collocazione, ma è funzionale all'inquadramento di un altro personaggio, Giuliano Vanghetti, in modo diverso 'precursore' in campo chirurgico, in quella chirurgia ortopedica che, nell'Ottocento, era diventata un campo tragicamente attuale.

È doveroso a questo proposito ringraziare sia il collega Dott. Mario M. D'Elios, per la collaborazione scientifica, sia la signora Giuliana Santini, erede di Giuliano Vanghetti, che ha conservato preziosamente il materiale del nonno.

L'ortopedia, come branca autonoma della chirurgia, aveva ricevuto grande impulso alla fine del XVIII secolo, ma già in passato il chirurgo si era dovuto confrontare con la necessità di ripristinare una funzione menomata, tramite il reimpianto protesico, soprattutto dopo la diffusione delle armi da fuoco. 
Nella seconda metà dell'Ottocento, questa esigenza era aumentata, alla luce soprattutto di nuove acquisizioni nel campo della cinematica e della fisiologia muscolare.

Se Chiarugi aveva, infatti, assorbito gli insegnamenti di Cullen e di Brown, in relazione alla fisiologia del sistema nervoso, che avevano un punto di riferimento ineludibile nel concetto di reattività di Albrecht von Haller, Vanghetti condivide questa riflessione, nel momento in cui indica la possibilità di recuperare l'energia del muscolo mozzato durante un'amputazione, per trasmetterla alla protesi applicata.

Vanghetti non era un chirurgo e, per questo, si rivolse ai chirurghi per realizzare sull'uomo quello che aveva sperimentato solo sugli animali.

Ebbe un colloquio con Putti e con Codivilla, dell'Istituto Ortopedico Rizzoli di Bologna, con Ceci, a Pisa, dove ebbe l'opportunità di applicare una protesi a un operaio, ferito sul lavoro, e l'intervento riuscì, ma non ci fu la tanto attesa svolta professionale.

Proprio nella tipografia Traversari di Empoli, Vanghetti, nel 1906, pubblicava un altro lavoro, dal titolo Plastica e protesi cinematiche.

La prima guerra mondiale segnò la svolta nella storia della chirurgia protesica, che dovette affrontare il problema dei numerosissimi reduci mutilati.

Il chirurgo tedesco Sauerbruch, dopo aver letto gli scritti di Vanghetti, se ne impossessò, iniziando ad applicare a tappeto la sua cura.

Forte della sua fama e delle evidenze raccolte da Vanghetti, rivendicò a sé la paternità di queste scoperte, finché, nel 1949, nove anni dopo la morte di Vanghetti stesso, fu cacciato dall'ospedale, in quanto la sclerosi cerebrale lo aveva trasformato in un pericoloso omicida.

Resta il fatto che il metodo di Vanghetti aveva trovato applicazione, grazie alla fama di Sauerbruch, che lo fece proprio.

L'opera dello sfortunato chirurgo empolese rimane, comunque, un turning point nella concezione della chirurgia ortopedica ricostruttiva: il principio della restituito ad integrum era un sogno, infatti, vagheggiato sin dalla chirurgia rinascimentale.

Siamo negli anni cinquanta del Novecento: medicina e chirurgia hanno conosciuto uno sviluppo impensato, grazie, in parte, a quei pionieri che avevano aperto la strada.

Né Chiarugi, né Vanghetti avevano lavorato in una struttura ospedaliera della loro città: l'uno aveva esercitato a Firenze, l'altro aveva anticipato i tempi a tal punto che non era stato possibile seguirlo; si avverte, quindi, rispetto a quel periodo, una frattura profonda tra l'istituzione sanitaria e l'uomo di scienza, tra l'ospedale e il medico innovatore.

Nel corso di questi ultimi decenni, invece, si è verificato il salto di qualità: questo iato si è venuto progressivamente colmando e tra il piano della assistenza e quello della ricerca non esiste più una cesura.

Il collante, l'elemento, cioè, che ha svolto la funzione di unificatore è da rintracciare, sicuramente, nell'opzione formativa, nel senso che il momento didattico ha rappresentato il luogo di incontro delle suggestioni scientifiche con le esigenze assistenziali.

A partire da alcuni anni, infatti, gli ospedali del comprensorio empolese sono stati sede elettiva del tirocinio teorico-pratico del personale infermieristico e, nel periodo a noi più vicino, anche del momento formativo, in quanto ad Empoli hanno trovato 
sede molti Corsi di Laurea decentrati dell'Area Sanitaria, potenziati sia dal sistema $e$ learning di apprendimento a distanza, sia dalla valorizzazione di competenze locali, che hanno trovato un ruolo attivo in questa prospettiva.

Dall'anno accademico 1996/1997, infatti, ad Empoli ha avuto sede il Corso di Diploma in Scienze Infermieristiche, poi convertito in Corso di Laurea Triennale e, dall'anno accademico 2002/2003, sono stati attivati anche il Corso di Laurea Triennale per Fisioterapisti, quello per Ostetriche e quello per Tecnici della Prevenzione.

Non a caso, la prima segreteria attiva per gli studenti, tra i vari corsi decentrati, ha avuto sede in Empoli, fornendo un utile ausilio organizzativo.

Oggi l'ospedale San Giuseppe è sede di un Centro di Fitoterapia, che ha caratteristiche pressoché uniche in Italia, e i nosocomi del comprensorio svolgono, spesso in strutture di carattere storico, un ruolo di primo piano dal punto di vista formativo.

\section{BIBLIOGRAFIA}

Bucchi, G., Guida di Empoli illustrata, Tip. Domenicana, Firenze 1916.

Cabras, P.L., Campanini, E., Lippi, D., Uno psichiatra prima della psichiatria. Vincenzio Chiarugi ed il Trattato "Della parte in genere ed in specie" (1793-1794), OIC, Firenze 1994.

Delitala, F., La vita e l'opera di Giuliano Vanghetti, «La Chirurgia degli Organi di Movimento", 28, 1942, pp. 1-13.

Lazzeri, L., Storia di Empoli, Tip. Monti, Empoli 1873.

Lippi, D., Considerazioni inedite da un manoscritto di V. Chiarugi, «RHMP», 1990, pp. 69-73.

Lippi, D., Campanini, E., Nel privato di una grande vita. L'epistolario di Vincenzio Chiarugi, psichiatra, Vecchiarelli, Roma 1992.

Pellegrini, G., Giuliano Vanghetti, «La Chirurgia degli Organi di Movimento», 26, 1940, pp. 1-4.

Siemoni, W., La Chiesa ed il Convento di S. Stefano degli Agostiniani a Empoli, «Storia della Valdelsa» (miscellanea), 7, 1986, pp. 40-42. 
I

IL

7 


\title{
Il polo Empolese universitario e le sue prospettive
}

\author{
di Alberto Magnaghi*
}

\section{PREMESSA}

Il rapporto università-territorio si modifica profondamente nella trasformazione del sistema socio-produttivo dal fordismo al postfordismo. Nell'organizzazione fordista della produzione dove prevalgono la produzione quantitativa, omologata, seriale e la standardizzazione dei prodotti, il rapporto fra sistema produttivo e territorio è di tipo strumentale: il territorio è un mero supporto di sistemi produttivi esogeni, i comuni offrono localizzazioni e servizi in cambio di occupazione e reddito.

Nel postfordismo prevale la domanda di produzione qualitativa, di differenziazione, unicità e tipicità dei prodotti. Il territorio interviene con $\mathrm{i}$ suoi giacimenti patrimoniali locali (ambientali, territoriali, culturali, artistici, ecc.) che divengono risorse per la produzione di 'valore aggiunto territoriale'. Questo nuovo ruolo delle specificità patrimoniali dei luoghi attribuisce nuovi ruoli agli enti territoriali nel promuovere il capitale fisso socio-territoriale: qualità ambientale, urbana, paesistica, del milieu socioculturale locale. In questo percorso diviene essenziale la valorizzazione della conoscenza e della formazione per lo sviluppo locale. I comuni attrezzano il territorio per elevarne la qualità e chiedono formazione, ricerca, innovazione. La domanda di università per il territorio si caratterizza in questa direzione. Non più solo capannoni, ma aule e laboratori.

\section{L'UNIVERSITÀ PER IL TERRITORIO ${ }^{1}$}

In questo nuovo contesto l'Università può diventare un vero e proprio incubatore e motore di sviluppo locale, attraverso la valorizzazione delle risorse patrimoniali locali,

* Professore Ordinario di Pianificazione territoriale, Università di Firenze; Presidente del Corso di laurea in Urbanistica e pianificazione territoriale e ambientale (Empoli).

${ }^{1}$ Questo paragrafo riprende in parte la mia relazione Per una Toscana delle Toscane: il decentramento dell'Università come fattore di sviluppo dei sistemi territoriali locali, tenuta nell'ambito 
nella formazione della forza lavoro per la società dell'informazione e della conoscenza, nella ricerca per l'innovazione dei processi produttivi per lo sviluppo locale.

In relazione ai 'modelli idealtipici' che hanno guidato le esperienze di decentramento territoriale, sia in Toscana che altrove (decentramento metropolitano, sedi 'clonate', distance learning, campus, decentramento diffuso unidirezionale, sistema reticolare multipolare), ${ }^{2}$ l'Ateneo fiorentino si è mosso inizialmente entro un modello di decentramento funzionale metropolitano, adottato prevalentemente da atenei ove la pressione dell'Università nella città storica risultava ormai eccessiva e altamente congestionante (es. Milano, Roma). Il problema di tale modello (entro cui si sono sviluppati nel tempo il Polo Biomedico di Careggi, il Polo Scientifico e Tecnologico di Sesto, il Polo delle Scienze Sociali di Novoli) è che spesso allontana solo di pochi chilometri il problema della congestione, provocando peraltro costi di urbanizzazione e di infrastrutturazione molto alti (edificazione ex novo, costo delle aree fabbricabili, accessibilità, servizi, ecc.), senza promuovere una riformulazione del ruolo urbano, economico e sociale dell'Università nel territorio, contribuendo altresì ad impoverire la città storica di funzioni pubbliche di terziario avanzato.

Nel frattempo, è tuttavia cresciuta in Toscana una forte domanda di attività universitarie nei diversi sistemi territoriali locali; l'insediamento di servizi rari di ricerca e formazione è infatti sempre più interpretato dalle amministrazioni locali-coerentemente con una più ampia riflessione anche nazionale e internazionale sul ruolo dell'Università- come potenziale motore di rafforzamento o conversione verso modelli di sviluppo locale, fondati sulla valorizzazione delle peculiarità e dei giacimenti patrimoniali del territorio. ${ }^{3}$

La politica urbanistica ed edilizia dell'Università diventa quindi un motore importante della pianificazione socioeconomica e territoriale volta alla valorizzazione delle identità delle 'molte Toscane', sul cui sviluppo peculiare si basa la produzione di futura ricchezza durevole, come indica il Piano di Sviluppo della Regione Toscana (2003).

La crescente e riconosciuta diversificazione e specializzazione del sistema delle economie locali regionali, delle sue variegate dotazioni patrimoniali ambientali, culturali,

del convegno promosso dall'Ateneo fiorentino Università e territorio: decentramento dell'Ateneo nella Provincia di Firenze, Firenze, 23 gennaio 2004.

${ }^{2}$ Una disamina critica dei diversi modelli e un'ipotesi di riorganizzazione territoriale multipolare e reticolare del modello universitario toscano si trova in Fanfano, D., L'Università del territorio, Alinea, Firenze 2001.

${ }^{3}$ Si veda al riguardo il cosiddetto 'Manifesto di Oviedo': Universitas e civitas, che definisce un 'decalogo' di punti programmatici da perseguire per ridefinire la relazione fra Università e territorio in termini di innovazione del capitale sociale, sviluppo endogeno e sostenibile: Gutierrez, R., Fermin Villeneuve, R., Il 'manifesto di Oviedo': Universitas et civitas. Il ruolo delle università nei processi di sviluppo territoriale, «Sviluppo locale», III, 16, pp. 120-129, Rosemberg \& Sellier, Torino 2001; sul tema della diffusione dei servizi rari e della 'territorializzazione' del sistema universitario come strumento di riequilibrio regionale vedasi anche: Magnaghi, A., Il progetto locale, Bollati Boringhieri, Torino 2000. Da segnalare anche il rilevante filone di ricerca sul tema delle learning regions sviluppato in particolare dal Center for Urban and Regional Development Studies (CURDS) dell'Università di Newcastle Upon Tyne e coordinato da John Goddard. 
produttive e di servizio ${ }^{4}$ pone infatti in maniera sempre più chiara la questione della capacità di riprodurre e potenziare a livello locale i saperi contestuali ed il patrimonio territoriale che costituisce il vero e proprio 'motore dello sviluppo' locale.

Nel sostegno di questo processo riproduttivo è evidente il nuovo ruolo che si pone per l'Università: la 'tutela e valorizzazione delle diversità locali' auspicata dalle ricerche sui sistemi territoriali locali e i loro giacimenti patrimoniali -nella prospettiva del rafforzamento del sistema regionale- si appoggia sull'esistenza di una solida base di 'agenzie' in grado: a) di riprodurre localmente le condizioni ed i patrimoni cognitivi che costituiscono l'elemento generatore specifico anche delle attività economiche; b) di mantenere attiva la relazione fra sapere codificato (scientifico) e sapere comune (contestuale) che garantisce l'innovazione del milieu locale. Un indebolimento di questo legame fra conoscenza, territorio e sviluppo comporta il rischio del venire meno di quella dimensione 'attiva' della territorialità che garantisce l'autosostenibilità locale dello sviluppo, rispetto alla prospettiva di sfruttamento e degrado di sistemi locali 'passivi' rispetto alle congiunture delle dinamiche esogene. ${ }^{5}$

Obiettivi prestazionali di questa natura non appaiono, anche sulla scorta di esperienze già avviate anche in altre regioni, perseguibili attraverso i tradizionali modelli di decentramento universitario. Ognuno dei modelli più sperimentati, dal decentramento metropolitano all'Università 'per cloni' - che gemma in maniera tendenzialmente indifferenziata discipline già presenti a livello di sede madre-, pone problemi relativi all'incerto legame con le caratteristiche del territorio in cui si colloca e, in particolare, rispetto alla capacità di migliorare globalmente l'ambiente e il patrimonio territoriale locale interpretandoli come risorsa per lo sviluppo endogeno.

$\grave{E}$ in questo contesto che l'Ateneo fiorentino ha avviato da qualche anno una seconda fase del decentramento, che chiamiamo di territorializzazione dell'Università adottando un modello di sistema policentrico e reticolare. Il modello ipotizza, attraverso le 'economie di scopo', consentite dalle tecnologie telematiche ed informatiche, di strutturare un sistema discreto di poli universitari locali ove le tipologie didattiche (e di ricerca) siano connesse in una relazione di reciprocità e mutua utilità alle specificità dell'ambiente socio-economico e fisico locale. $\mathrm{Ne}$ discende un sistema dai contenuti didattici complessi e 'localmente orientati' ove ciascuna sede è strutturata in forme didattiche e di ricerca che consentono anche attività differenziate e specifiche (laboratori di ricerca sul campo, stage, diffusione territoriale di specifici livelli formativi e di ricerca, ecc.) che sostanziano questo rapporto attivo con il contesto.

L'intenso legame che questo tipo di modello -seppure nella sua versione idealtipicasi orienta a costituire con il territorio e con le sue risorse, sviluppa in definitiva e tende a rafforzare una specifica polarità prestazionale che sembra prefigurarsi come strategica

${ }^{4} \mathrm{Si}$ veda al riguardo l'insieme di ricerche condotte recentemente dall'IRPET sulla diversificazione dei sistemi economici locali e sui diversi profili socio economici che essi sono venuti assumendo. Si tratta di fatto di una letteratura che evidenzia la complessità di relazioni fra base locale per l'esportazione, scambi interni alla regione e relazioni di tipo sovralocale generata dalla relativa specializzazione ed identità dei diversi sistemi locali. I lavori di riferimento da questo punto di vista sono: Cavalieri, A., Toscana e toscane, IRPET-F. Angeli, Milano 1999, e Bacci, L., Sistemi locali in Toscana. Modelli e percorsi territoriali dello sviluppo regionale, IRPET-F. Angeli, Milano 2002. 
per un rinnovato ruolo dell'Università nella società e che riguarda il compito del supporto e di cooperazione allo sviluppo locale. Tale ruolo richiede, per essere praticato, la costituzione di un complesso ed ampio spettro di centri e servizi in grado di interagire con il capitale sociale e territoriale e con gli attori socio economici ed in grado di funzionare come 'antenne' di flussi informativi e tematici 'da' e 'per' il territorio. ${ }^{6}$

I caratteri innovativi e strategici di questo modello regionale "territorializzato" possono riassumere nei seguenti:

- la connessione a rete dell'intero sistema consente ad ogni polo di fruire di servizi didattici (tra cui e-learning) e di strutture di eccellenza dell'intero Ateneo, e viceversa consente all'Ateneo di arricchire il proprio patrimonio e la propria peculiarità attraverso la valorizzazione dei singoli giacimenti culturali locali;

- la funzione di incubatore di sviluppo locale consente al singolo polo di produrre 'valore aggiunto territoriale', ovvero di produrre innovazione sociale ed economica attraverso la messa in valore del patrimonio socio-territoriale locale, di saperi 'contestuali' (locali o di rete regionale) e la formazione di competenze e di ricerche mirate a questo fine; ${ }^{8}$ sul piano sociale ed economico tale modello può utilmente appoggiarsi su forme di partnership pubblico-privato che rafforzano la coerenza interna del sistema locale e, al tempo stesso, producono un importante radicamento ed innalzamento culturale opposto ai fenomeni di 'migrazione culturale' e di abbandono degli studi che una sede universitaria 'remota' induce sugli utenti di aree periferiche;

- l'Università territorializzata eleva il rango delle città in rete, indipendentemente dalla loro dimensione;

- la struttura policentrica aumenta accessibilità e diritto allo studio per le aree periferiche e marginali della regione, costituendo nuovi bacini di utenza, decongestionando il polo centrale; ${ }^{9}$

${ }^{5}$ Sul concetto di 'territorialità attiva' in relazione allo sviluppo dei sistemi territoriali locali si veda Dematteis, G., Per una geografia della territorialità attiva, in Bonora P. (a cura di) SLoT quaderno 1, Baskerville, Bologna 2001.

${ }^{6}$ I punti 5 e 6 del citato manifesto di Oviedo focalizzano a tale riguardo l'attenzione sulla costituzione di 'centri o istituti per la cooperazione allo sviluppo territoriale' e sulla diffusione ed accrescimento di 'centri di trasferimento tematico' che hanno il compito di integrare le tradizionali attività di formazione e ricerca universitaria ampliando il campo della interazione con gli attori e le esigenze degli attori locali.

${ }^{7}$ L'avvio e lo sviluppo dei 'poli' di Prato, Empoli, Pistoia, delle sedi di Scandicci, Calenzano, S. Casciano, Figline, Vaglia, l'avvio di analoghi poli per gli atenei di Pisa e Siena (Lucca, Livorno, Arezzo, Grosseto), ma anche la diffusa offerta insediativa in atto da parte di enti locali (S. Giovanni Valdarno, Calenzano, Quarrata, Colle Valdelsa, Livorno, ecc.), richiedono in futuro un'attenta programmazione della distribuzione funzionale e territoriale delle attività, dato il significato strategico che va assumendo questo secondo modello di decentramento.

${ }^{8}$ Ad esempio nel Piano locale di sviluppo del Circondario Empolese Valdelsa (IRPET, 2002), il Polo Universitario è visto come uno dei motori dello sviluppo nella riorganizzazione del sistema produttivo multisettoriale, in particolare nella attivazione delle nuove filiere legate al ciclo agricoltura-turismo-ambiente-cultura.

${ }^{9}$ E stata verificata peraltro, a livello regionale, la stretta relazione fra abbandoni e distanza dal centro universitario della abitazione degli studenti. Si veda al riguardo Compagnino, A., 
- l'offerta di sedi, servizi e attrezzature da parte delle amministrazioni locali (solitamente in aree e edifici dismessi di pregio in posizioni urbane centrali ad alta accessibilità pubblica, es. l'ex ospedale di Empoli; o di alto pregio architettonico, es. la villa di Castelpulci a Scandicci) riduce o azzera i costi di insediamento per l'Università; favorisce la rivitalizzazione del patrimonio delle città storiche medie e piccole della Toscana, accrescendone il rango con l'attivazione di servizi rari alla persona e all'impresa; si appoggia a infrastrutture di trasporto pubblico esistenti, contribuendo al loro potenziamento;

- il contributo economico degli enti territoriali (sia in forma di contributi diretti sia coinvolgendo l'Università nelle attività socio economiche e culturali del territorio) consente in prospettiva di dotare i poli di una relativa autonomia finanziaria e di personale, garantendone la continuità, la qualità della formazione e della ricerca, radicando così in maniera efficace le attività di formazione universitaria al milieu territoriale ed istituzionale locale, superando il pendolarismo dei docenti e l'estraneità al contesto che affligge le sedi 'clonate' dalla sede centrale.

\section{Il Polo Universitario Empolese: un laboratorio per lo sViluppo della "Toscana delle Toscane".}

Rispetto a questo percorso di territorializzazione delle università toscane il Polo Universitario Empolese può specificamente caratterizzarsi come incubatore per la riqualificazione socioproduttiva di un sistema territoriale locale a economia complessa e integrata, ad alta qualità paesistica e ambientale e artistica.

La presenza nel polo di molti corsi di laurea (Medicina-Infermieristica, Ottica, Chimica applicata, Tossicologia dell'ambiente, Urbanistica e Pianificazione territoriale ambientale, Ingegneria gestionale, Economia aziendale) che vanno consolidando la loro presenza territoriale consente di attivare sia relazioni specifiche di settore sia con gli enti pubblici ${ }^{10}$ che con le singole aziende ${ }^{11}$ e associazioni (imprenditoriali, sindacali,

Gori, E., L'università in Toscana, evoluzione problemi e prospettive, IRPET-F. Angeli, Milano 1995.

${ }^{10}$ Un esempio in questa direzione è costituito dal Corso di Laurea di Urbanistica e Pianificazione Territoriale e Ambientale che contribuisce, con le attività dei suoi laboratori didattici, alla costruzione del SIT del Circondario Empolese Val d'Elsa, qualificandolo con la costruzione di un "Atlante del patrimonio territoriale e ambientale" in collaborazione con l'IRPET e con il Circondario, utile al Piano di sviluppo del Circondario stesso; organizza il tirocinio degli studenti del modulo professionalizzante in Cartografia Tematica, attivato con la Regione, negli uffici tecnici del Circondario e dei Comuni; costruisce un atlante socioeconomico e della progettualità sociale, per l'implementazione delle conferenze d'area sul piano di sviluppo locale; attiva diverse convenzioni su progetti di valorizzazione del territorio e dell'ambiente, quali ad esempio il Master plan per il Parco dell'Arno, della Bassa Val d'Elsa e della bassa Val di Pesa.

${ }^{11}$ Ad esempio le intense e qualificate relazioni del Corso di laurea di Medicina con la ASL, le relazioni di consulenze formazione, ricerca dei corsi di Ingegneria e Chimica con aziende e associazioni dell'area e gli studi sul sistema economico locale. 
sociali, come già sta avvenendo), sia di ipotizzare in futuro una relazione organica fra il Polo Universitario Empolese e le problematiche socioeconomiche, territoriali e ambientali del Circondario.

I giacimenti patrimoniali che caratterizzano il Circondario, e che possono essere oggetto di promozione e messa in valore con il concorso dell'Università si possono sintetizzare nei seguenti:

- un insieme di sistemi ambientali di alta qualità: le colline delle colture pregiate della vite e dell'ulivo, la montagna del Montalbano con le sue articolate pendici boscate e sentieristiche, le valli dell'Arno, dell'Elsa, della Pesa e dei loro sottobacini, costituenti un reticolo idrografico di grande potenzialità come corridoi ecologici e attività fruitive, sportive e turistiche;

- sistemi territoriali a forte identità storica e culturale, costituiti da un fitto reticolo, integrato in sottosistemi policentrici di piccole e medie città storiche di grande pregio artistico, culturale e di produzioni tipiche (Empoli- Fucecchio-Montelupo, Vinci-Cerreto, Guidi-Capraia e Limite, Gambassi-Montaione, CertaldoCastelfiorentino-Montespertoli);

- i sistemi produttivi: un sistema multisettoriale aperto (vetro, ceramica, cornici, legno tessile-abbigliamento, alimentaristica, cuoio, calzature, pelletteria...) ad alto valore aggiunto artigiano ed artistico; cui si aggiungono le potenzialità di un 'distretto rurale' e di 'eccellenza agroalimentare' (vino, olio, tartufo, formaggio...) e di un turismo culturale, ambientale, enogastronomico che può avvalersi di una dotazione patrimoniale ingente e articolata: un paesaggio agrario collinare e vallivo (il sottosistema del Montalbano, il nodo orografico Montespertoli, Fiano, Certaldo, la media valle dell'Arno, la bassa Val d'Elsa) di grande valore estetico, storico, ambientale;

- emergenza di nuove filiere integrate quali quella relativa a agricoltura-ambienteturismo-cultura, e di terziario di servizio che possono connettere in modo integrato i valori ambientali, territoriali e paesistici con la tradizionale produzione artigianale, valorizzandone la qualità e attivando nuove reti di commercializzazione;

- il capitale sociale: forte presenza di reti civiche, di associazionismo, di volontariato, di imprese sociali, di saperi diffusi (nel campo artigianale e artistico, ambiente, dell'assistenza, della solidarietà, della cultura, dei servizi...).

Il Circondario Empolese Valdelsa rappresenta istituzionalmente uno dei sistemi territoriali locali sui quali si appoggia "la Toscana delle Toscane", ovvero il progetto del Piano Regionale di Sviluppo che vede nelle tante peculiarità dei luoghi i giacimenti patrimoniali per uno sviluppo sostenibile.

Il Polo di Empoli è dunque esemplificativo di un sistema di relazioni che si possono attivare con un sistema socioeconomico ad alta complessità, articolato territorialmente in sub-sistemi territoriali locali dotati di specifiche identità, di un'alta integrazione produttiva a forte valenza artigianale e artistica, di una forte valenza agroalimentare di eccellenza, di centri urbani di alto livello artistico culturale, e di una forte coesione sociale. 


\section{NuOVI RUOLI DEL GOVERNO LOCALE PER RIQUALIFICARE LO SVILUPPO}

Per quanto riguarda i modelli di sviluppo, la crisi e l'evoluzione dei sistemi economici locali, in particolare dei distretti della prima generazione, nel quadro della globalizzazione dei mercati, ha ormai indotto in molte amministrazioni locali la diffusa consapevolezza, che la risposta locale (regionale e subregionale) non può che muoversi nella ricerca della eccellenza delle produzioni di valore aggiunto legato alla qualità e alla tipicità, ma soprattutto della unicità del proprio 'stile di sviluppo' nello scambio internazionale attraverso la piena valorizzazione dei giacimenti patrimoniali locali, pena la delocalizzazione della produzione di beni in regioni a minori costi salariali, ambientali, sociali. Questa unicità consente di impostare sistemi produttivi che scambiano beni irriproducibili altrove: in questo sta il loro 'valore aggiunto territoriale' rispetto ai mercati globali. Di qui il cambiamento di ruolo del territorio da supporto delle attività produttive a produttore di risorse rare per qualificare il modello di sviluppo.

Il modello che meglio affronta questa trasformazione è il modello di sviluppo per sistemi territoriali locali caratterizzati da sistemi produttivi multidistrettuali integrati, fondati sulla valorizzazione dei giacimenti patrimoniali locali in forme durevoli e autosostenibili.

La complessità multisettoriale del sistema produttivo locale empolese, costituisce in questa prospettiva una risorsa adatta a mettere in valore sinergicamente tutti gli aspetti del patrimonio locale (e non un aspetto a discapito degli altri come sovente è successo nel modello fordista e nei modelli distrettuali di prima generazione); la complessità socioproduttiva del sistema locale è la chiave della durevolezza dello sviluppo, nel senso che consente nel tempo al sistema locale gli adattamenti necessari all'evoluzione turbolenta del sistema globale senza perdere la propria identità, come rischia di avvenire nei sistemi distrettuali monosettoriali.

Per perseguire questo modello il compito del governo locale (Circondario e Comuni) si configura: a) come denotazione e rappresentazione socialmente condivisa del patrimonio locale (ambientale, territoriale, culturale, sociale), fino a definire lo "statuto del territorio", ${ }^{12}$ ovvero le regole di trasformazione del patrimonio stesso in quanto risorse che ne consentano la riproducibilità e l'arricchimento nel tempo; b) come attivazione di istituti di partecipazione degli attori sociali per delineare uno scenario di futuro condiviso per la messa in valore integrata delle risorse territoriali individuate nello statuto; con ciò attivando un patto tra gli attori, ognuno con i suoi compiti specifici (e limiti di compatibilità delle azioni) per la realizzazione e la gestione dello scenario condiviso.

In questa prospettiva l'ente locale assume funzioni nuove di governo dell'economia, se si assume il compito di selezionare, promuovere e valorizzare la formazione di imprese e sistemi di imprese che producono beni sinergici con la valorizzazione del patrimonio.

${ }^{12}$ Vedasi in proposito il rilievo attribuito dalla nuova legge di governo del territorio della Regione Toscana (1/2005) alla parte identitaria e statutaria dei piani con lo sviluppo dei concetti (già presenti nella LR 5/95) di 'risorse essenziali del territorio', di 'invarianti strutturali' e di 'statuto del territorio'. 
D'altra parte la crisi dei distretti monofunzionali e la complessità intersettoriale dei processi di valorizzazione del patrimonio territoriale (pensiamo alla trasversalità e integrazione della filiera agricoltura di qualità e tipica-ambiente-paesaggio-turismo-cultura) richiede approcci integrati alla pianificazione; i distretti devono essere pensati come filiere complesse, integrate, multisettoriali, il cui 'prodotto' non è valutato per settore, ma per 'valore aggiunto territoriale'; occorre di conseguenza concepire un distretto produttivo integrato (che è capace di integrare attività agricole, artigianali, industriali, turistiche, commerciali, terziarie avanzate).

Infine è necessario curare la qualità degli insediamenti produttivi (che sono oggi una delle cause principali di degrado ambientale, territoriale, paesistico, distretti compresi), dal momento che la qualità territoriale e paesistica costituisce uno degli elementi qualificanti un modello di sviluppo fondato sulla valorizzazione del patrimonio; occorre porre dei criteri valutativi (es. aree ecologicamente attrezzate) che certifichino l'insediamento dal punto di vista ambientale (acqua aria, suolo, rifiuti, ecosistemi), della qualità urbanistica (minimizzare gli impatti funzionali e paesistici, elevare la qualità urbana complessiva), e della qualità edilizia (riduzione dell'impatto ambientale e paesistico, tipologie edilizie, materiali e modalità costruttive coerenti con i caratteri paesistici locali).

\section{I COMPITI DEl Polo Universitario}

Se la complessità e l' integrazione delle filiere del modello economico locale in rapporto attivo con le risorse territoriali sono gli elementi alla base della capacità di autoriproduzione futura del sistema, lo sono altrettanto i processi di innovazione, conoscenza, formazione, ricerca, progetto. In questo processo notevole importanza acquisisce il Polo Universitario che interviene, oltre che nella formazione (sempre più importante nel rinnovamento qualitativo del sistema economico manifatturiero e delle sue componenti di terziario avanzato), anche nella ricerca, nell' innovazione, nell' aiuto al sistema locale a sviluppare le nuove filiere di eccellenza connesse alla valorizzazione del patrimonio.

In sintesi i ruoli del Polo nei confronti del territorio possono essere:

- produrre conoscenza, progetti e processi formativi per la valorizzazione del patrimonio ambientale, territoriale e sociale locale;

- produrre conoscenze progetti e processi formativi per la sicurezza ambientale, la salute e il benessere;

- produrre servizi e progetti di innovazione tecnologica e organizzativa per il sistema economico produttivo multisettoriale e integrato;

- attivare laboratori specialistici per il trasferimento tecnologico alle imprese;

- produrre formazione articolata ai diversi livelli (diplomi, lauree specialistiche, stage, master, dottorati, centri di formazione, ecc.), mirata alla riqualificazione e specializzazione della forza lavoro per cicli produttivi di alta qualità e tipici, per le funzioni complesse di governo del territorio ai diversi livelli. 
Per assolvere a queste complesse funzioni di 'incubatore' di sviluppo locale il Polo Universitario ha bisogno di sviluppare alcune funzioni essenziali:

- innanzitutto costituirsi non come somma di corsi di laurea 'clonati' dall'Università di Firenze, ma come sistema organico di competenze, con una sua identità ed eccellenza, per trattare i problemi dello sviluppo locale del Circondario; ciò richiede che il Polo si costituisca come progetto culturale, formativo e di ricerca, attraverso un processo che veda impegnati i corsi di laurea delle diverse Facoltà in un dialogo reciproco ${ }^{13}$ e con gli attori del Circondario per mettere a fuoco e organizzare il progetto stesso;

- dotarsi di personale universitario, docente e non docente, radicato nel Polo, per superare una fase di 'pendolarismo' e rendere stabili le relazioni con il territorio;

- dotarsi di strutture di ricerca con forti componenti multidisciplinari, che siano in grado di attivare relazioni produttive con i diversi ambiti e attori del territorio, pubblici e privati;

- dotarsi di un sistema di spazi adeguati alla formazione, alla ricerca, alle relazioni con la città e il territorio (valorizzazione della città storica, dei trasporti pubblici, dei luoghi della cultura) $;^{14}$

- essere dotato di una sufficiente autonomia economica e gestionale;

- essere sostenuto da una struttura locale di attori pubblici e privati interessati all'innovazione e allo sviluppo locale. Al di là della forma specifica che assumerà questa struttura ${ }^{15}$ essa deve procedere dalla costruzione di un 'patto per lo sviluppo locale' fra attori socioeconomici, enti pubblici e Università in cui il governo del processo formativo, della ricerca e della produzione culturale viene vissuto e praticato, nell'epoca della società e dell'economia della conoscenza, come un problema vitale del territorio e del suo sviluppo.

${ }^{13}$ Attivando ad esempio un Centro Servizi di Ateneo, o un Comitato Scientifico Didattico come parte della struttura di gestione del Polo.

${ }^{14}$ Rispetto a questo obiettivo la scelta del Circondario di utilizzare per il Polo Universitario e i suoi servizi un sistema integrato di edifici della città storica (l'ex Convento degli Agostiniani, l'ospedale, il Palazzo delle esposizioni, l'ex circondario, gli edifici aggregati alla stazione ferroviaria, ecc.) è destinata a produrre qualificazione funzionale e culturale della città stessa di Empoli contribuendo ad elevarne il rango nel sistema regionale (vedasi su questo argomento la relazione del Prof. Marco Massa in questo stesso volume).

${ }^{15}$ Ad esempio a Prato il PIN/Consorzio Ingegneria Prato) vede compresenti soci pubblici e privati: Università di Firenze, Camera di Commercio di Prato, Unione Industriale, Serin (Unione Industriale), Associazioni artigiane, SATA (Confartigianato), Fondazione Cariprato, Comune di Prato, Provincia di Prato, Provincia di Pistoia, Consiag, Co.pr.a.s. Analoga struttura presenta l'UNISER del Polo Universitario di Pistoia, che aggiunge al Consiglio di Amministrazione una struttura di Comitato Scientifico-didattico. 
I

IL

7 


\title{
Il programma di sviluppo del Polo universitario e il riuso dell'ex ospedale di San Giuseppe
}

\author{
di Marco Massa
}

Il mio intervento illustra il lavoro che da due anni sto svolgendo con gli studenti del Laboratorio di progettazione urbanistica sul tema della sede del Polo universitario. ${ }^{1}$

Il Polo universitario di Empoli è nato da un accordo fra enti locali e università che prevedeva una decina di corsi di laurea, (delle Facoltà di Medicina, Architetttura, Ingegneria, Scienze matematiche fisiche e naturali, Economia e Commercio) selezionati in relazione alle caratteristiche e alle esigenze della realtà produttiva e sociale locale. Molti di questi corsi si sono installati e l'università è oggi una presenza significativa nella vita locale.

La rapidità della crescita del Polo non è priva di inconvenienti e disagi, dovuti all'inefficiente distribuzione delle sedi e alla scarsità degli spazi e dei servizi (si veda la planimetria con le sedi attuali: figura 1); la situazione è tale che di recente l'ASL ha deciso autonomamente di localizzare in un'area dismessa del comune di Vinci la propria attività formativa (istituzionalmente autonoma ma connessa con i corsi di laurea di medicina).

A questo punto è necessario consolidare e qualificare le funzioni universitarie con un vero e proprio progetto/programma di sviluppo edilizio e di integrazione delle diverse sedi fra loro e nella struttura della città e del territorio. Il lavoro del Laboratorio ha preso corpo su questo obiettivo. Dai risultati potranno essere eventualmente tratte indicazioni per le politiche urbanistiche istituzionali che dovranno quanto prima mettere a punto una strategia appropriata.

* Professore Ordinario di Urbanistica, Coordinatore del Laboratorio di progettazione urbanistica del Corso di laurea in Urbanistica e pianificazione territoriale e ambientale.

${ }^{1}$ Il laboratorio è collocato al terzo anno del cdl e nell'Anno Accademico 2003-2004 si compone del corso base di Urbanistica (M. Massa); dei moduli di Recupero e riqualificazione urbana (G. Giovannelli); Estimo e valutazione dei piani urbanistici (C. Borocci); Pianificazione degli insediamenti (F.M. Allegretti). Il lavoro si è avvalso anche di una verifica dell'accessibilità al polo e della riqualificazione dell'asse Spicchio-stazione eseguito nell'ambito del corso di Pianificazione dei trasporti e delle infrastrutture (M. Facchinetti). Gli studenti del Laboratorio che hanno lavorato su questo tema sono: per l'Anno Accademico 2003/2004, J. Bennati; M. Caroti; F. Ghergo; D. Jacopetti; N. Martino; C. Pagliai; S. Ramerini; per l'Anno Accademico 2002/ 2003, L. Aquili; S. Maccari; M. Mameli; M. Tredici; C. Albertosi; F. Bavoni; L. Leonardi; M. Umiliani.

Il programma di sviluppo edilizio

L'INNOVAZIONE PER LO SVILUPPO LOCALE 


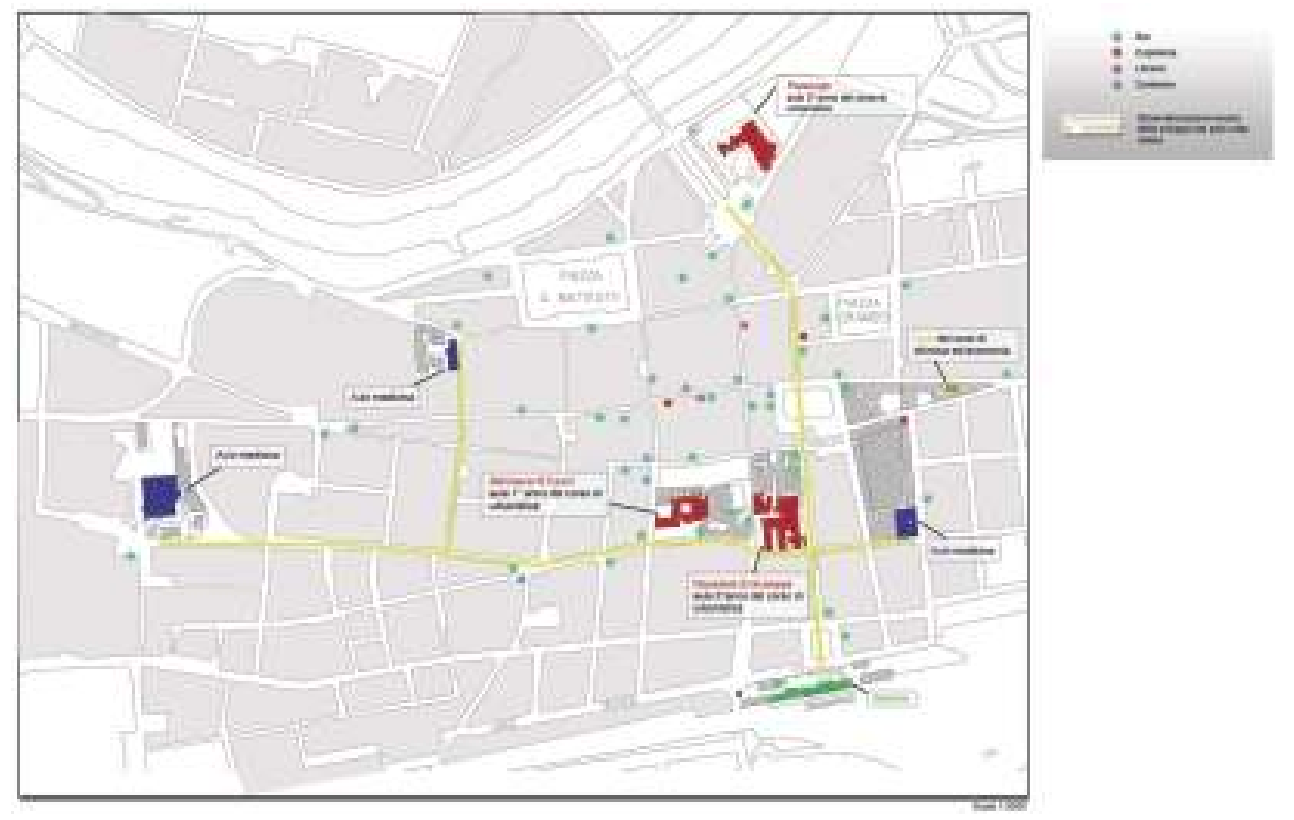

Figura 1 - Le sedi attuali del Polo universitario nel tessuto urbano.

In un primo momento, dopo aver preso in considerazione e scartato l'ipotesi di un campus esterno alla città, il lavoro si è concentrato sul riuso dell'ospedale di San Giuseppe di cui si prevedeva entro pochi anni la totale dismissione, come unica sede di tutti i corsi di laurea; su tale ipotesi si era infatti realizzato un consenso da parte delle istituzioni implicate. Peraltro, una piccola parte dell'ospedale è stata subito messa a disposizione per ospitare aule di alcuni corsi di laurea.

Le ragioni della convenienza di questa scelta sono evidenti, per il circondario e per il Polo.

Per il Polo universitario, sono l'accessibilità dai mezzi pubblici, in particolare dalla stazione ferroviaria, la collocazione lungo il principale asse urbano che consente la disponibilità di numerosi servizi, come biblioteche, servizi di ristorazione, ecc., il rapporto di 'comunicazione' diretta con la città e gli abitanti.

Per il territorio sono da un lato le ricadute socio-economiche di questo insediamento, sotto diversi aspetti (miglioramento del rango urbano e dell'offerta di lavoro specializzato, sviluppo di nuove attività economiche legate alla presenza di studenti e docenti, ecc.), ma anche gli effetti di prestigio per l'immagine urbana che derivano dalla valorizzazione di un monumento attualmente trascurato e dal ruolo dell'università come una componente dell'identità di Empoli e del circondario.

\section{LA VALUTAZIONE DELLA DOMANDA DI SPAZI DIDATTICI}

Il lavoro si è mosso su due binari: da un lato la valutazione della 'domanda' di spazi espressa dall'insieme dei corsi di laurea; dall'altro la valutazione delle caratteristiche 
della 'offerta' di spazi disponibili espressa sopratutto, ma non solo, dall'ospedale di San Giuseppe. Il progetto/programma è scaturito dal confronto ragionato fra queste due valutazioni.

La valutazione della 'domanda' considera essenzialmente il numero di studenti prevedibili rispetto ai quali, applicando gli standard seguiti dall'Università di Firenze per il proprio 'piano edilizio', si può stimare il fabbisogno di spazio. ${ }^{2}$ Tale valutazione è stata un processo non lineare, dato che di recente si sono manifestate consistenti $\mathrm{e}$ nuove esigenze sopratutto nella Facoltà di Medicina e che di conseguenza si è attivata una intensa riflessione da parte delle istituzioni con implicazioni sulla scelta delle sedi.

\begin{tabular}{|l|c|}
\hline Corso di Laurea & Iscritti $\boldsymbol{n}$. \\
\hline $\begin{array}{l}\text { Scienze infermieristiche, ostetricia, fisioterapia, tecniche } \\
\text { della prevenzione in ambienti e luoghi di lavoro }\end{array}$ & 446 \\
\hline Urbanistica e pianificazione ambientale & 250 \\
\hline Chimica applicata e ottica & 150 \\
\hline Ingegneria gestionale & 25 \\
\hline Economia & 25 \\
\hline TOTALE & $\mathbf{8 9 6}$ \\
\hline
\end{tabular}

Tabella 1 - Studenti iscritti nell'A.A. 2003/2004.

\begin{tabular}{|c|c|c|}
\hline \multirow{2}{*}{$\begin{array}{l}\quad \text { Facoltà } \\
\text { Faceltà di Se.Matematiche, } \\
\text { Fisiche e Naturali }\end{array}$} & Corso di laurea & previsioni \\
\hline & Chimica & 25 \\
\hline \multirow{2}{*}{ Facoltà di Ingegneria } & Ingegneria gestionale (laurea specialistica) & 30 \\
\hline & $\begin{array}{l}\text { Econoenia Axiendale of le piccole e medie } \\
\text { imprese) }\end{array}$ & 60 \\
\hline \multirow{2}{*}{$\begin{array}{l}\text { Fucoltà di Economia } \\
\text { Facolts di Arehitettura }\end{array}$} & Urbanistica e Pianificazicne Territoriale & 220 \\
\hline & Lauree Specialistiche & 80 \\
\hline \multirow[t]{6}{*}{ Facoltà di Medijcina e Chirurgin } & Infermieristica & \\
\hline & Fisioterapia & \\
\hline & Ostetricia & \\
\hline & Tecniche della Prevenzione nell'ambiente & \\
\hline & e nei laoghi di lavoro & \\
\hline & Totale laures mediche & 1100 \\
\hline \multirow[t]{2}{*}{ TOTALE COMPLESSIVO } & & 1515 \\
\hline & Master & 300 \\
\hline
\end{tabular}

Tabella 2 - Previsioni di sviluppo al 2010.

${ }^{2}$ Gli standard utilizzati per la didattica misurano $4,5 \mathrm{~m}^{2}$ per studente $(2,6$ per aule e 1,9 per connettivo/servizi). Va sottolineato che tali standard sono applicati a edifici di nuova costruzione (come Novoli). In questo calcolo non si considera il fabbisogno di spazi per la ricerca, ossia di spazi dipartimentali, localizzati nelle sedi di Firenze; si può ipotizzare tuttavia che uffici per svolgere ricerche nel polo siano disponibili nell'ambito della quota di spazi adibita a servizi. 
Comunque, rispetto alla situazione degli iscritti nell'Anno Accademico 2003/2004 (complessivamente circa 900), nel breve periodo di 4-6 anni si può ipotizzare, sulla base di colloqui con i responsabili dei diversi corsi di laurea e delle tendenze degli ultimi due anni, una crescita di 600 ulteriori studenti, senza contare i master (che sono circa 300, cfr. tabelle 1 e 2). Questa crescita è sostenuta principalmente da medicina cha passa dal 50\% attuale al 70\% degli iscritti. Sono i corsi di Scienze infermieristiche che per il momento fanno di Empoli un polo di attrazione a livello nazionale.

C’è poi un'altra struttura di rilevanti dimensioni (il 'centro di formazione' dell'ASL, che accoglie più di 1.000 persone), al momento non coordinata coi programmi del Polo; tale struttura, accanto all'insufficienza degli spazi disponibili in Empoli, è un'altra delle spinte all'origine della scelta di insediare tutte le attività di formazione dell'ASL a Spicchio.

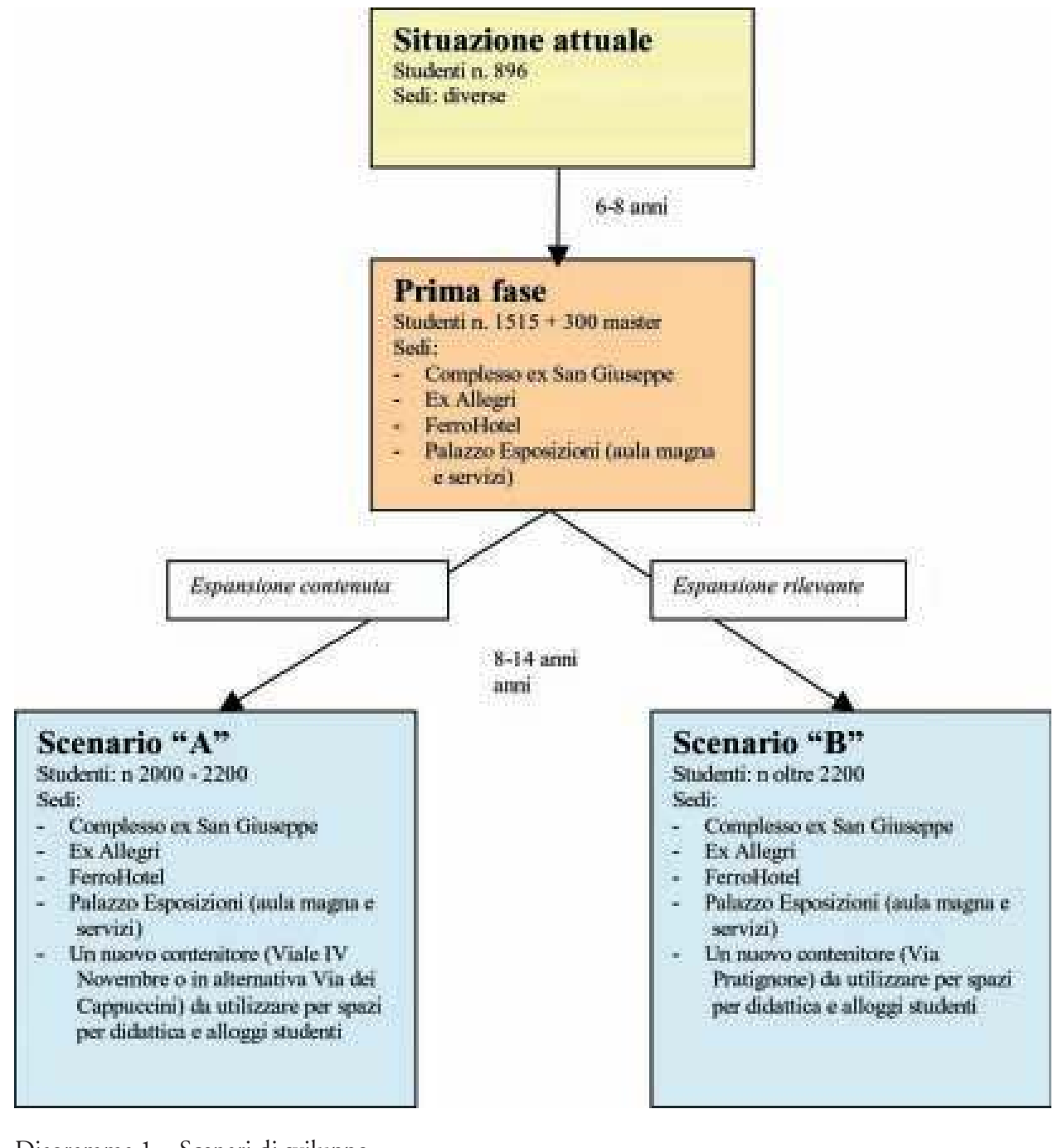

Diagramma 1 - Scenari di sviluppo. 
Occorre fare un'altra riflessione sul periodo successivo. Si danno infatti due possibilità: può darsi che il numero di studenti continui a crescere, oppure che la crescita rallenti. Per sintonizzare le previsioni del programma di sviluppo del Polo con la durata del Piano regolatore (dieci anni) occorre considerare anche le due possibili direzioni di crescita (vedi diagramma 1 'Scenari di sviluppo').

Applicando gli standard dell'Università di Firenze si è calcolato il fabbisogno complessivo del Polo per il breve periodo: circa $6.900 \mathrm{~m}^{2}$ per spazi didattici ai quali sono da aggiungere gli incrementi per i master e i servizi comuni extradidattici (servizi amministrativi, aula magna, ecc.), per un totale di circa $8.500 \mathrm{~m}^{2}$.

Utilizzando poi uno studio svolto dalla ASL si è costruita una prima ipotesi di individuazione del numero e del 'taglio' dimensionale delle aule (tabelle 3 e 4).

\begin{tabular}{|c|c|c|}
\hline LOCALIE & NUMARO & CARATTERISTICHE \\
\hline \multicolumn{3}{|l|}{ Corsi facoltu di medicina } \\
\hline Aule & 10 & Da 50 posti \\
\hline Aule & 3 & Ds 100 posti \\
\hline Aule & 6 & $D_{n} 40$ posti \\
\hline Sale per laveri di grappo & 6 & $\begin{array}{l}\text { Da } 20 \text { posti , con tavoli eireolari } \\
\text { Da } 30 \text { posti cen } 4 \text { salette laterali per lavori df } \\
\text { gruppo }\end{array}$ \\
\hline Aule qualificazioni & 4 & $\mathrm{Da} 40$ posti \\
\hline Aula multimediale & 4 & Da 50 posti \\
\hline Laboratori faule dimastraxioni & 8 & Da 30 posti (90 me ciascuna) \\
\hline Palestru attremata per eserciá fisioterapici & 1 & $\mathrm{D}=60 \mathrm{mg}$ \\
\hline Laboratorio addestramsento emergenza & 3 & Da 30 posti \\
\hline Laboratorio linguistico & 1 & Da 30 posti \\
\hline $\begin{array}{l}\text { Laboratorio di chimica (secondo normative } \\
\text { per spazi sicureza e decontaminazione) e } \\
\text { fisica }\end{array}$ & 2 & Da 40 posti \\
\hline Sale riunioni & 2 & Da 40 posti \\
\hline Portincria e centralino & & I postazione \\
\hline Front-office & & 2 postazioni \\
\hline BIBLIOTECA : & I & 100 postarioni Jettura \\
\hline Ufficio & 1 & 2 postazioni \\
\hline Sale lettura e pastazioni PC & 4 & Da 30 persane \\
\hline \multicolumn{3}{|l|}{ UFFICI: } \\
\hline & $\begin{array}{l}8 \mathrm{da} \text { due } \\
\text { possi }\end{array}$ & Per 15 amministrativi \\
\hline & $\begin{array}{l}6 \text { per due } \\
\text { persone }\end{array}$ & Pet presidenti, e docensi universitari \\
\hline & $\begin{array}{l}8 \text { per tre } \\
\text { persone }\end{array}$ & Tutor \\
\hline & $\begin{array}{l}1 \text { per due } \\
\text { persone }\end{array}$ & Ufficio coentabilitả \\
\hline & $\begin{array}{l}4 \text { per due } \\
\text { persone }\end{array}$ & Uflicio master e specializaxzioni \\
\hline & $\begin{array}{l}4 \text { per tre } \\
\text { persone }\end{array}$ & Uffici di Direzione \\
\hline \multicolumn{3}{|l|}{ Punto ristoro Bur } \\
\hline Spogliatoio dipendenti & 1 & Per 10 persone \\
\hline Spogliatoio studenti & 5 & Per 50 persoen \\
\hline Guardaroba e deposito divise & 1 & $50 \mathrm{mg}$ \\
\hline Sala medicalinfermeria & 1 & \\
\hline deposito attrezmiture & 3 & \\
\hline
\end{tabular}

Tabella 3 - Fabbisogno di spazi (per numero e superficie delle aule) Corsi di Laurea della Facoltà di Medicina. 


\begin{tabular}{|l|l|l}
\hline $\begin{array}{l}\text { LOCALE } \\
\text { Corso Urbanistica e } \\
\text { pianificazione territoriale e } \\
\text { Ambientale } \\
\text { Aule }\end{array}$ & NLMERO & CARATTERISTCHE \\
\hline Aule & 2 & \\
\hline Sala conputer & 5 & Grandi \\
\hline Corsi Facoltà di Economia & 1 & modie \\
\hline Aule & & \\
\hline $\begin{array}{l}\text { Aula prof. } \\
\text { Aula studenti }\end{array}$ & 2 & 50 posti \\
\hline Corsi Facoltà di Ingegneria & 1 & \\
\hline Aule & 1 & \\
\hline $\begin{array}{l}\text { Corsi facoltà di scienze } \\
\text { matematiche - chimica }\end{array}$ & 2 & Medie da 40 posti \\
\hline nule & & 1 postazione \\
\hline
\end{tabular}

Tabella 4 - Fabbisogno di spazi (per numero e superficie delle aule) Altri corsi di Laurea.

Infine, un'altra ricaduta importante di questa forte crescita concerne l'alloggio degli studenti. Oggi infatti circa 130 studenti soprattutto di Scienze infermieristiche, provenienti da luoghi di residenza extraregionali, risiedono in sedi reperite dall'ASL a Montaione e Fucecchio, con tempi di trasferimento giornalieri consistenti, difficoltà di accesso alle strutture universitarie in relazione al numero limitato di servizi di trasporto pubblico e con costi e disagi pertanto elevati. A fronte dell'ipotesi di un'ulteriore crescita occorre definire una politica che combinando interventi diretti e indiretti riesca a soddisfare almeno una parte di questa consistente domanda se si vuole offrire un insediamento apprezzato e ricercato dagli studenti, dai docenti e dai ricercatori italiani e stranieri non solo per l'offerta curriculare qualificata, ma anche per le condizioni di vita e di studio.

\section{LA VALUTAZIONE DELL'OFFERTA DI SPAZI DIDATTICI:}

\section{L'INDIVIDUAZIONE DELLE SEDI POSSIBILI}

Per quanto riguarda la 'offerta' degli spazi disponibili, le valutazioni riguardano l'ospedale (che è comunque il perno del progetto di localizzazione del Polo) e le altre sedi possibili nel periodo dei dieci anni di validità del Piano regolatore, distinguendo le due fasi di cui si è detto. Infatti, per le quantità attuali e le prospettive di cui si è detto l'ospedale si rivela insufficiente.

L'ospedale è un organismo di grande importanza per la storia della città, degradato da trasformazioni improprie. Occorreva dunque ricostruirne la storia facendo attenzione a individuarne $\mathrm{i}$ caratteri alterati da ripristinare. Naturalmente la lettura critica che abbiamo fatto vale come metodo più che nel merito non avendo potuto utilizzare gli strumenti appropriati a una precisa ricerca scientifica (ricerche d'archivio, saggi sulle murature, rilievi metrici esatti, ecc.). 
La tavola allegata fuori testo riassume la storia che abbiamo ricostruito attraverso la lettura delle planimetrie d'epoca e degli altri documenti disponibili. Si tratta di quattro tappe fondamentali del processo evolutivo che corrispondono alle maggiori trasformazioni.

La prima tappa documenta lo stato dell'ospedale all'epoca d'impianto (metà Settecento): due alti stanzoni (piano terra a due livelli e primo piano) costruiti a ridosso delle mura collegati ad una palazzina e al 'torrino' su via Paladini. Il corpo principale, in gran parte ancora oggi riconoscibile, è un edificio a forma di " $U$ " a carattere monumentale, dalla semplice planimetria d'impianto simmetrico, dall'architettura di chiara ispirazione mediceo-lorenese, con murature intonacate di bianco su cui spiccano le ampie finestre incorniciate di pietra serena.

La seconda tappa documenta gli inizi dell'espansione col prolungamento verso sud delle ali laterali proseguendo lo stesso impianto e lo stesso 'stile' architettonico. Fino a questa fase (1840 circa) le addizioni e le trasformazioni si sono inscritte in un certo senso nel processo evolutivo dell'impianto originario, di per sé in contrasto col disegno delle mura e del bastione, ma sempre mantenendo la riconoscibilità dei due manufatti (l'ospedale e il bastione), come due oggetti distinti accostati che conservano il carattere monumentale dell'insieme. In seguito l'ospedale fagocita la forma delle fortificazioni, degradando, fra l'altro, proprio questo carattere.

Infatti la fine dell'Ottocento e i primi decenni del Novecento (terza tappa) vedono una crescita sconnessa senza un progetto complessivo, con l'apertura di via Roma che ha tagliato il bastione; la costruzione di una nuova stecca su via Roma, la cui testata su via G. da Empoli si distingue per il linguaggio architettonico più curato e simile agli edifici precedenti; la costruzione di nuovi edifici su via Paladini; pesanti ristrutturazioni interne con la frammentazione degli stanzoni a doppia altezza.

Nel periodo successivo, fino ad oggi (quarta tappa), continua il processo di trasformazione incrementale che ha portato alla saturazione delle corti con numerose superfetazioni (per lo più impianti tecnici); la sopraelevazione della stecca su via Roma; nuovi solai nelle due ali a doppia altezza, con lo stravolgimento delle aperture (alcune delle quali sono state chiuse); ulteriori ristrutturazioni e suddivisioni interne che hanno snaturato il complesso (figura 2 'Assonometria dello stato attuale del complesso di San Giuseppe').

Sovrapponendo le mappe dei diversi periodi è stato possibile datare le diverse parti e individuare le superfetazioni, le parti storiche da salvaguardare, i tracciati di impianto e le parti che meritano la ricostruzione o il ripristino rispetto a quelle che invece necessitano di demolizione (perché in contrasto con l'impianto storico).

Questa lettura è preliminare alle scelte di progetto se si vuole dare priorità alla qualità del recupero e serve a individuare le funzioni compatibili con le caratteristiche spaziali delle varie parti dell'edificio a partire da queste ultime. Si tratta di un metodo che rovescia quello spesso seguito che altera i caratteri spaziali per adattarli alle esigenze funzionali dei programmi.

Parallelamente, è stata compiuta un'indagine sulle principali sedi universitarie e sugli edifici dismessi in prossimità della stazione suscettibili di utilizzo per funzioni connesse al Polo. Per fare ciò si sono utilizzate informazioni desunte dal Piano regolatore e dagli uffici tecnici comunali. Le sedi vagliate sono: il complesso di viale IV Novem- 
bre; quello di via dei Cappuccini; il complesso ex Montevivo; il FerroHotel (edificio di proprietà delle FS, posto accanto alla stazione, già usato come foresteria dalle FS stesse); il Palazzo delle Esposizioni (utilizzato oggi come sede universitaria); il complesso ex Allegri in località Spicchio (sul quale sono in corso i lavori di adattamento a sede delle attività di formazione dell'ASL).

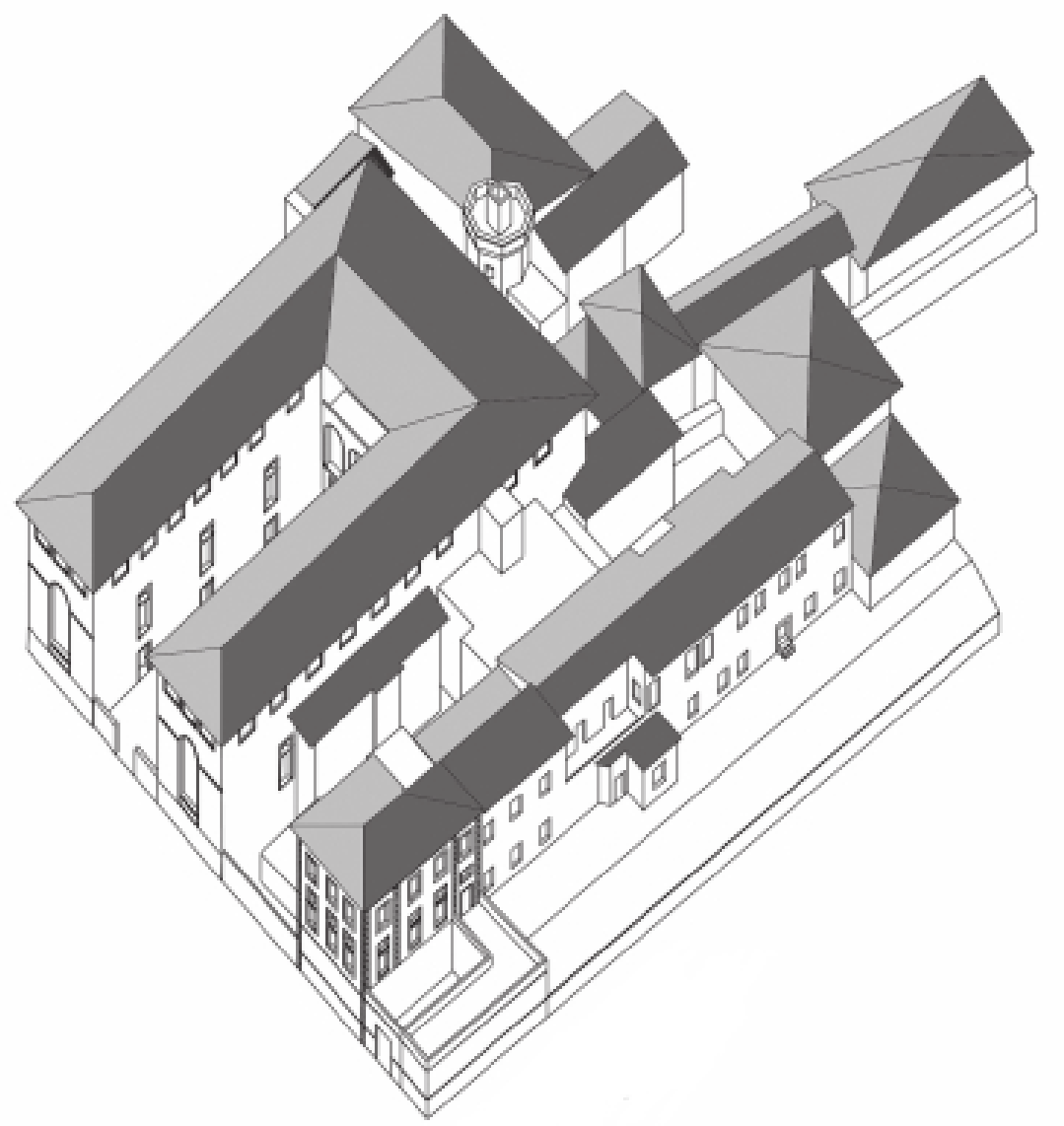

Figura 2 - Assonometria dello stato attuale del complesso di San Giuseppe.

\section{IL PROGRAMMA EDILIZIO, LE FASI E GLI 'SCENARI'}

Alla luce dell'insieme di queste riflessioni, si è reso necessario riconsiderare la localizzazione del Polo rispetto all'ipotesi di partenza che lo vedeva concentrato nell'ospedale, e riconoscere una localizzazione policentrica capace di assicurare puntuale risposta in termini di spazi e di servizi alle esigenze dei diversi corsi di studio, adeguata flessibilità al fine di far fronte all'evoluzione futura della domanda, ma mantenendo fermi alcuni requisiti fondamentali, quali il ruolo determinante dell'ospedale, la prossimità alla stazione ferroviaria, la stretta relazione col centro urbano. 
Già allo stato attuale, considerate le scelte prese, si sta delineando un asse ordinatore fra la stazione e la località Spicchio che associa le diverse strutture (dall'ex Allegri, al Palazzo delle esposizioni, all'ospedale). Si tratta quindi di razionalizzare tale asse, lungo il quale l'ospedale di San Giuseppe si caratterizza per la posizione baricentrica.

In riferimento alla prima fase indicata nel diagramma che riassume gli scenari di sviluppo (con una previsione di 1530 studenti più 300 master), gli interventi e le scelte che si ritengono necessari sono:

- recupero dell'ospedale al fine di reperire spazi didattici di tutte le facoltà del Polo; il dimensionamento del complesso (circa $8.300 \mathrm{~m}^{2}$ ) è stimato a parte, nell'ambito di un progetto di recupero;

- utilizzo del complesso ex Allegri a Spicchio per i corsi di aggiornamento del personale e per i master e i corsi universitari di medicina nel periodo di esecuzione dei lavori di recupero dell'ospedale;

- utilizzo del Palazzo delle esposizioni per servizi generali del Polo e della città (aula magna e spazi per convegni, segreterie, ma anche albergo, giardini pubblici, ecc.);

- ristrutturazione dell'edificio ex FerroHotel per foresteria studenti; anche per questo edificio il dimensionamento (34 posti letto) è stimato a parte nell'ambito di un progetto di recupero.

Considerato che questo scenario prevede l'utilizzo pressoché esclusivo di edifici di proprietà pubblica (del comune di Empoli o di altri enti), si può ritenere che si caratterizzi per un'elevata fattibilità.

A seconda dell'entità di crescita del periodo successivo si distinguono poi due ulteriori scenari: il primo (A, espansione contenuta: figura 3), ha per obiettivo principale la realizzazione di un certo numero di alloggi da mettere a disposizione degli studenti oltre a reperire ulteriori spazi per la didattica. A queste esigenze risultano rispondere ambedue i complessi di viale IV Novembre e via dei Cappuccini; in particolare quest'ultimo risulta più conveniente dato che il Piano regolatore prevede già circa 3.000 $\mathrm{m}^{2}$ di residenza, corrispondenti a circa 250 posti letto per studenti. In questa ipotesi, la parte dell'ex Allegri che si libera per lo spostamento dei corsi di laurea di medicina potrebbe essere utilizzata a seconda delle necessità per ospitare altre residenze o spazi didattici (quali i master).

Il secondo scenario (B, espansione rilevante) presenta una domanda di spazi che può essere soddisfatta dall'area ex Montevivo, le cui dimensioni suggeriscono di pensare a un mix di funzioni pubblico-private sull'esempio dell'area di Novoli a Firenze.

\section{I PROGETTI PRELIMINARI: L’OSPEDALE}

Dei quattro interventi proposti nello scenario di prima fase due sono stati approfonditi a livello di 'progetto preliminare' (il recupero dell'ospedale e quello del FerroHotel). 

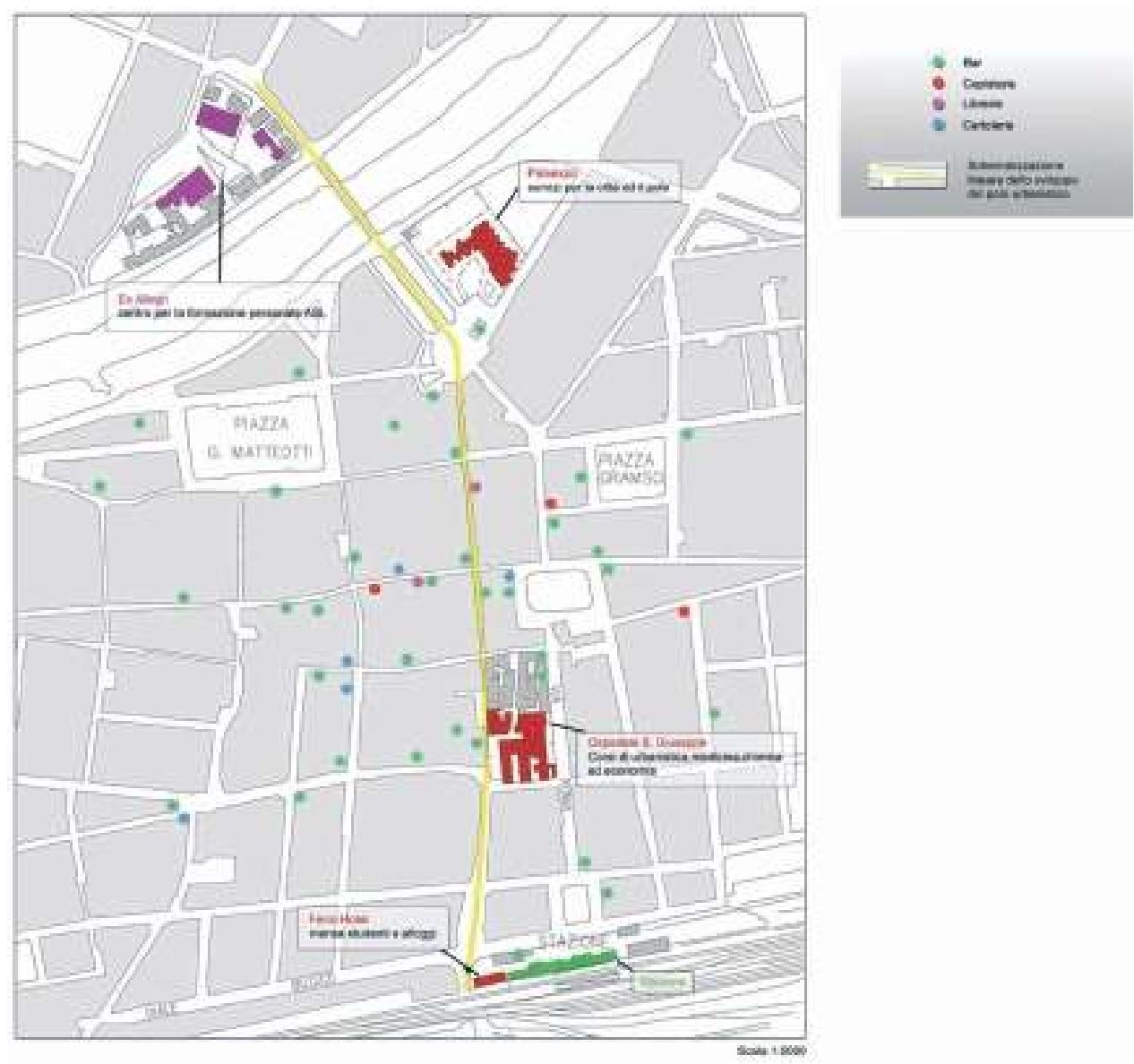

Figura 3 - Proposta urbanistica per il sistema delle sedi. Previsione prima fase.

Tale approfondimento è necessario per verificare in modo puntuale la capacità dell'ospedale di soddisfare la domanda di spazi didattici e il numero di posti letto ricavabili nel FerroHotel. Per brevità si riporta qui solo il progetto relativo al recupero dell'ex ospedale.

Tenendo presente le caratteristiche del corso di laurea in urbanistica il lavoro più che in un progetto univoco è consistito nella definizione di criteri e regole per addestrare gli studenti alla cultura del progetto di recupero, per collaborare, valutare, istruire programmi di recupero complessi, anche attraverso confronti ed esempi di casi analoghi.

Questo aspetto è avvalorato dal fatto che l'ospedale è un organismo di grandi dimensioni. Di conseguenza il progetto di riuso si caratterizza, per l'incidenza della componente urbanistica, piuttosto come un piano di recupero per un piccolo quartiere che distingue le diverse parti, i percorsi e i luoghi vocati allo scambio con la città. Il progetto di recupero così concepito è in definitiva assimilabile ad uno studio di fattibilità dal quale potranno eventualmente derivare progetti più esatti. 
In sintesi il progetto di recupero prevede il restauro del complesso storico (parte a U, 'torrino', testata dell'edificio in linea su via G. da Empoli); la ristrutturazione leggera dell'area circostante il 'torrino'; la demolizione e ricostruzione dei corpi di fabbrica su via Roma e su via Paladini. Le demolizioni/ricostruzioni, tendono oltre che a una miglior configurazione architettonica, a garantire una maggiore efficienza dell'utilizzo delle volumetrie e a rompere l'isolamento del complesso rispetto alla città.

\subsection{Permeabilità e spazi di connessione}

Un primo requisito del progetto di recupero infatti consiste nel dotarsi di un sistema di spazi pubblici o aperti di connessione con la città. In questo senso, sono da sottolineare almeno tre proposte:

- un nuovo importante percorso pedonale fra via Roma e via Fabiani, reso possibile dalla riconfigurazione dei volumi novecenteschi, che forma all'incrocio con via Paladini uno spazio ad uso di piazza pubblica antistante l'ingresso del Polo; il percorso supera il dislivello fra via Roma e via Paladini tramite una rampa;

- la riqualificazione delle corti su via G. da Empoli (una denominata 'del pozzo', a causa dell'antico pozzo esistente, l'altra "della palma", in ragione dell'albero col quale si propone di sostituire le attuali superfetazioni);

- la valorizzazione dei due 'slarghi' che via G. da Empoli origina all'incrocio rispettivamente con via Roma e via Fabiani (con nuovi ingressi al Polo, un bar su via Roma, arredo per la sosta e l'incontro).

Queste proposte rendono permeabile il complesso edilizio e al contempo aprono accessi al Polo da tutte le direzioni (figura 4 'Planimetria delle coperture').

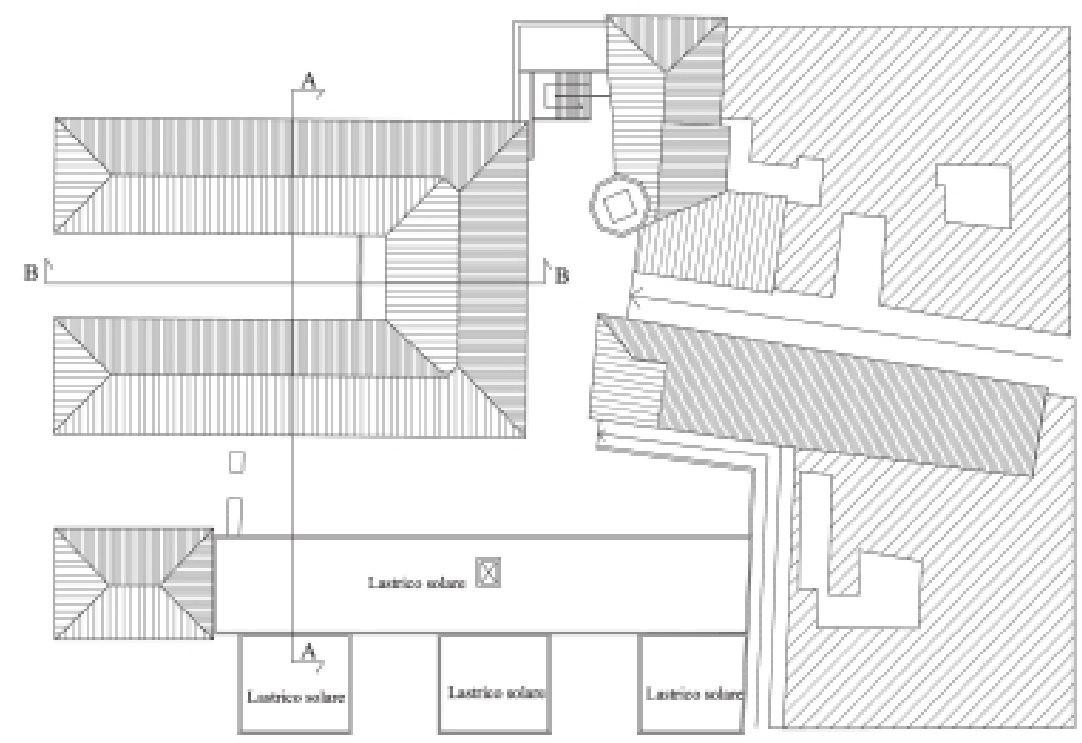

Figura 4 - Recupero del complesso di San Giuseppe. Planimetria coperture. 


\subsection{La memoria del bastione}

Circa il manufatto, in generale si può premettere che l'obiettivo cui si tende è di ripristinare la leggibilità (almeno come segno, come traccia evocativa) delle due strutture originarie da cui si è generato (l'ospedale e il bastione).

Per quanto riguarda il bastione, si suppone, con le informazioni disponibili, di rimettere in luce tratti della muratura su via G. da Empoli; mentre, dato che l'allineamento attuale del terrapieno su via Roma sembra non coincidente con quello originario del bastione stesso, su questo lato il nuovo corpo di fabbrica di cui si dirà evocherà comunque nell'architettura le caratteristiche dimensionali e figurative delle fortificazioni antiche.

\subsection{Conservazione delle parti storiche e ripristino delle facciate}

Il complesso architettonico dell'ex ospedale è destinato quasi interamente ad aule; la sua articolazione volumetrica consente di rendere visibili in sedi distinte i vari corsi di laurea: quelli di medicina sono nei tre corpi di fabbrica in linea; quello di urbanistica nell'area del 'torrino', mentre i corsi di laurea minori (economia, ingegneria, scienze) sono posti nella zona ex scuola infermieri opportunamente riconfigurata.

Gli interventi si distinguono a seconda del carattere del corpo di fabbrica. La porzione centrale del complesso, di più antica formazione, conserva elementi di qualità formale e strutturale tale da richiedere un restauro di tipo conservativo. Il progetto pertanto ipotizza l'eliminazione delle superfetazioni, il restauro-consolidamento delle coperture el'eliminazione dei solai intermedi in contrasto con l'impianto originario. In questo modo si potranno ripristinare le antiche aperture originarie (figura 5). Particolare attenzione è stata posta nella
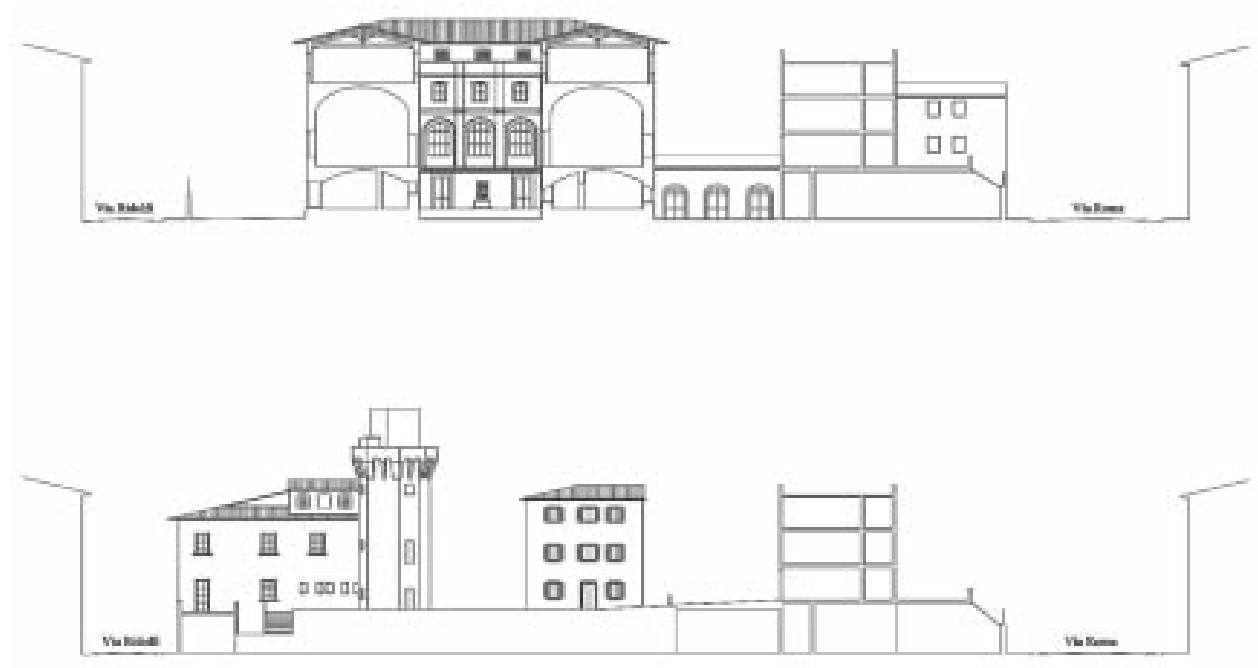

Figura 5 - Recupero del complesso di San Giuseppe. Sezioni.

delimitazione delle aule secondo moduli dimensionali compatibili con i caratteri strutturali e tipologici del complesso(figura 6). Pur con questa avvertenza è possibile corrispondere 
pressoché integralmente alle esigenze espresse dal programma ricordato dell'ASL, grazie anche alla ricostruzione dell'ala su via Roma (si veda la tabella 5 "Offerta di spazi, per numero e superficie di aule" a confronto con le precedenti tabelle 3 e 4 "Fabbisogno di spazi per numero e superficie di aule").

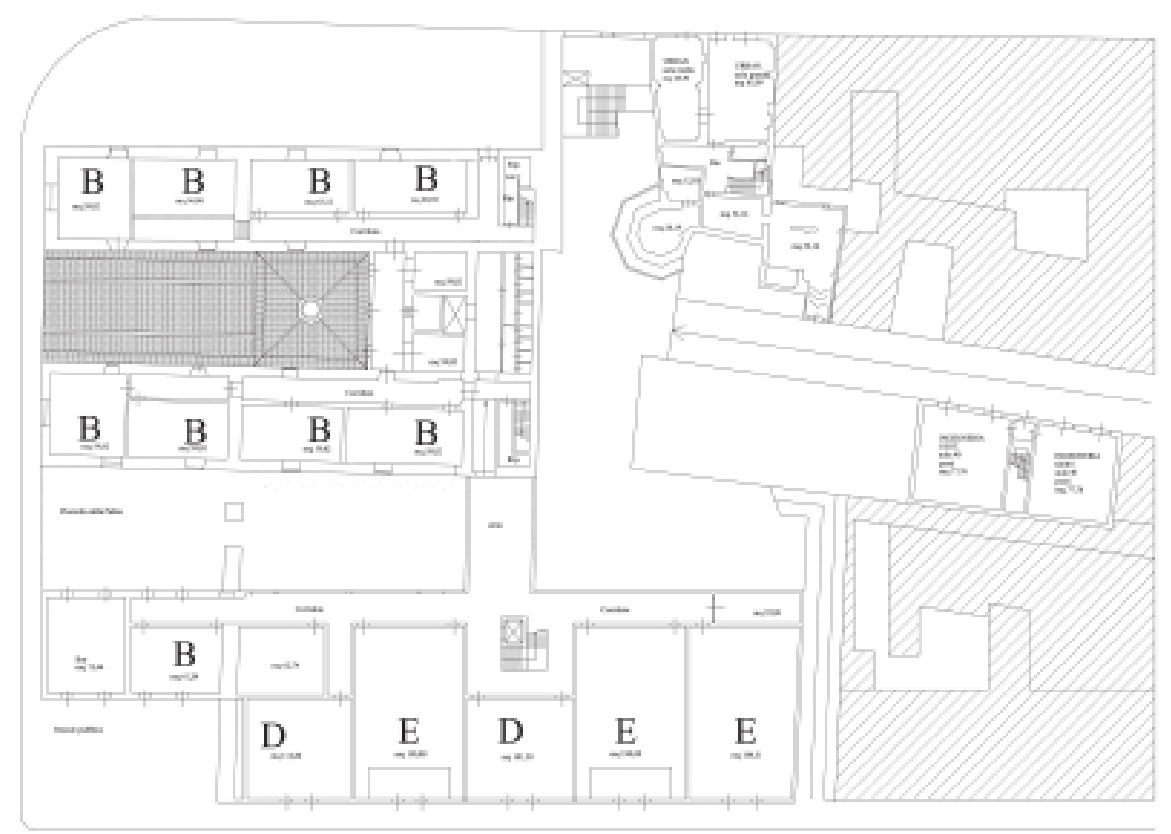

Figura 6 - Recupero del complesso di San Giuseppe. Layout primo piano.

\subsection{Ricostruzione dell'ala su via Roma}

L'intervento più impegnativo è pertanto quello sulla zona dell'ex bastione e dell'ala costruita nel 1939 su via Roma. Essendo priva di qualsiasi interesse storico architettonico, di cattiva qualità e degradata, se ne propone la demolizione, compreso il terrapieno che in parte la copre, e la sostituzione con un corpo di fabbrica di tre piani fuori terra più un piano seminterrato. Di tale corpo di fabbrica sono definiti gli allineamenti, le altezze, gli accessi, la configurazione che richiama l'immagine di un forte, l'organizzazione interna, e sono esplorati i primi effetti di inserimento nel paesaggio cittadino (si veda la figura 7 'Recupero del complesso di San Giuseppe. Assonometria' a confronto con la figura 2 'Assonometria dello stato attuale del complesso di San Giuseppe').

Con tale intervento si mette a disposizione una consistente superficie sulla quale è possibile ricavare le aule di tagli o caratteristiche non reperibili nel complesso storico.

\section{ConCLusioni}

A questo punto si possono trarre alcune conclusioni. Nell'insieme, il progetto propone un ridimensionamento della massa costruita del complesso, in particolare delle 
addizioni all'impianto storico: il volume attuale è di $46.300 \mathrm{~m}^{3}$, comprese le superfetazioni $\left(5.300 \mathrm{~m}^{3}\right)$; il progetto prevede, compresa la ricostruzione dell'ala su via Roma, un volume di $44.600 \mathrm{~m}^{3}$, con una riduzione di $1.700 \mathrm{~m}^{3}$.

\begin{tabular}{|c|c|c|}
\hline Focote o Corso do latreat & previsioni di progetito & esipenze \\
\hline & n. . ocal y carallentbote & in focal o cavodistische \\
\hline \multicolumn{3}{|l|}{$\begin{array}{l}\text { Corwo Urbaniatica o pianificazione tarritoriale o } \\
\text { Ambientale }\end{array}$} \\
\hline Aulo & 2 da 36 poet & 2 grand \\
\hline Aile & B da $27-33$ posti & 5 meds \\
\hline Ssla computer & 5 da 37 post & 1 mede \\
\hline \multicolumn{3}{|l|}{ Corsi Facoltis df Economia } \\
\hline Aule & 2 da 51 poed & 2 da 50 posti \\
\hline Aula doontif & $1 \mathrm{da} 15 \mathrm{posi}$ & 1 \\
\hline Aesa student & 1 da 17 poed & t \\
\hline \multicolumn{3}{|l|}{ Corsi Facolth id Ingegneria } \\
\hline Auve & $2 \mathrm{da} 41$ e 44 poet & 2 da 40 posti \\
\hline \multicolumn{3}{|l|}{ Corsi facolta di sciense matematiche - chimica. } \\
\hline aul & 2 da 33 e 41 posti & 2 da 30.40 posi \\
\hline \multicolumn{3}{|l|}{ Corsi facolsh di medicina } \\
\hline Alfe & 3 da 106 posti & 3 da tco post \\
\hline Aule & $10 \mathrm{da} 56$ posti & 10 da 00 post \\
\hline Alise & E da 40 posil & 64 a 40 posati \\
\hline Sale per lavon di orippo & 6 da20-25 pasti: & $\begin{array}{l}6 \text { do verti posti con tow } \\
\text { circotari }\end{array}$ \\
\hline Ause qualfeazioni & $4 \mathrm{da} 40$ post & 4 da 40 posti \\
\hline Aúa muhmodialo & 1 da50 posti & 4 da 50 posti \\
\hline Labcrateri laub dimeatrajoci & B da 30 poss & 8 da 30 posti $(00$ mal \\
\hline Palosira afliezzats pet oserdaz focleorspici & $1 \mathrm{da} 59 \mathrm{mq}$ & $1 \mathrm{da} 60 \mathrm{ma}$ \\
\hline Latcoratorio addewriemento emerpanza & 3 da 30 poess. & 9 ta 30 pesti \\
\hline Laboratorio lingustice & 1 da 30 poed & 1 da 30 posti \\
\hline 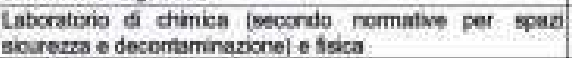 & 2 da 40 pows & $2 \mathrm{da} 40$ posti \\
\hline Salo ribuiboi & 2 de 40 poed & 2 da 40 posti \\
\hline OIBLIOTECA: & $1 \mathrm{da} 180 \mathrm{mq}$ & $\begin{array}{l}1 \text { da } 160 \text { postabon } \\
\text { ettura }\end{array}$ \\
\hline UKto : & Ida 2 postivioni & 1 per due postationi \\
\hline Sab letura o postason PC & 4 da 30 persone & 4 da 30 persone. \\
\hline \multirow[t]{2}{*}{ UFFICt } & $2 \mathrm{da} 21-35 \mathrm{mq}$ & 15 da due goes: \\
\hline & $3 \mathrm{di} 50 \mathrm{mq}$ & 12 da be posti \\
\hline Spogifutcis dipenderti & 1 da 30 parsone & 1 per 30 parscone \\
\hline Spoglabiolostudenti & 4 da 30 persone & 5 per 50 persone \\
\hline Quardandea edeposito tivise & tofa 62 ma & $1 \mathrm{tan} 50 \mathrm{mq}$ \\
\hline Sada madicolirlomeria & $1 \mathrm{da} 22 \mathrm{miq}$ & 1 \\
\hline depceto atrecerature & $4 \mathrm{du} 11.17 \mathrm{mg}$ & 3 \\
\hline
\end{tabular}

Tabella 5 - Progetto. Offerta di spazi rispetto alla domanda (numero e superficie di aule).

Inoltre la riconfigurazione del volume con la liberazione delle superfetazioni delle corti e la ricostruzione del fronte su via Roma permette la migliore leggibilità del complesso storico che riacquista l'aspetto monumentale che gli è proprio.

La superficie netta del complesso risulta di circa $8.300 \mathrm{~m}^{2}$. (si veda la tabella 6 'Progetto. Offerta di spazi complessiva (per corso di laurea)'); c'è quindi rispetto ai $6.800 \mathrm{~m}^{2}$ di fabbisogno stimati in base agli standard una maggiorazione dovuta in parte al fatto che i responsabili dei corsi di medicina hanno richiesto superfici consistenti per attività specifiche (laboratori, palestra, sale multimediali, ecc.) non conteggiati nel fabbisogno di spazi didattici; in parte al fatto che alcune di queste superfici sono in comune con altri 
corsi e quindi devono essere lievemente maggiorate (es. biblioteca, sala computer, ecc.); infine, si deve considerare che intervenendo su un edificio esistente di valore storico, si devono osservare vincoli che comportano essenzialmente un maggior sviluppo di spazi di connettivo. D'altra parte, il corso di laurea in urbanistica dispone di spazi leggermente inferiori allo standard in ragione della possibilità di usufruire di alcuni servizi in comune.

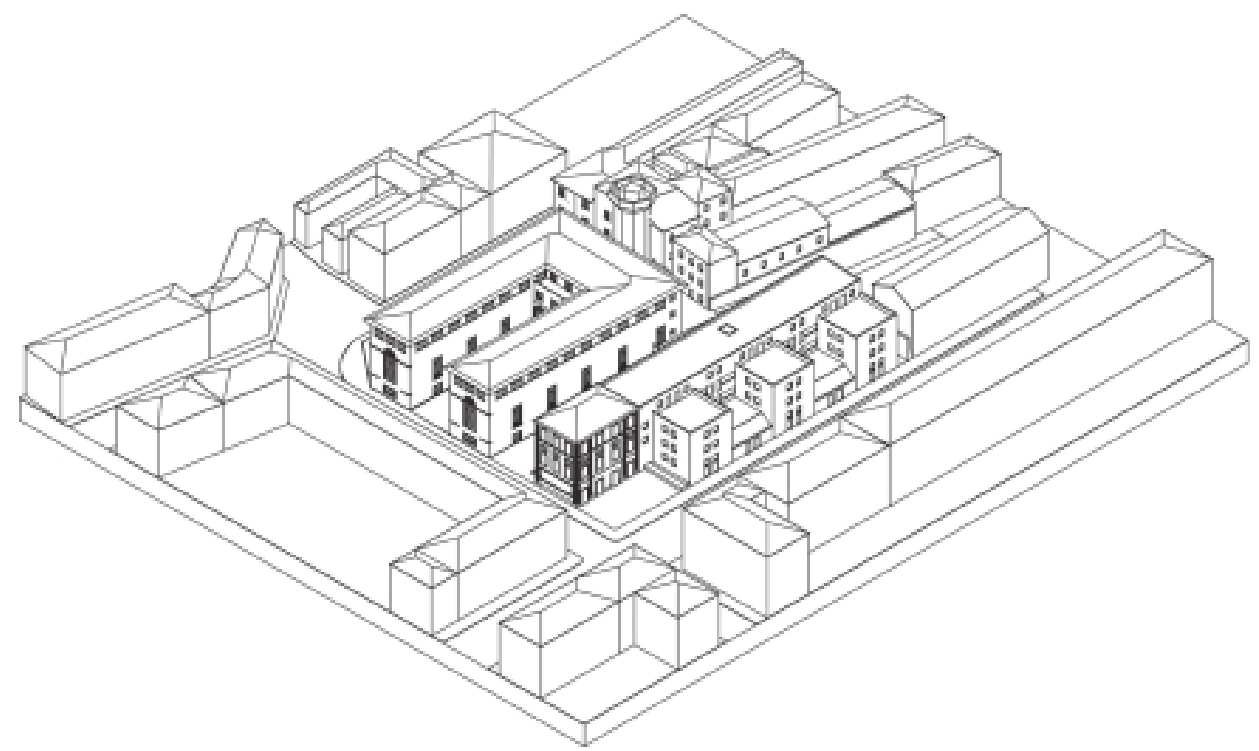

Figura 7 - Recupero del complesso di San Giuseppe. Assonometria.

\begin{tabular}{|l|r|r|r|r|}
\hline & \multicolumn{3}{|c|}{ Previsioni di progetto } & $\begin{array}{c}\text { standard } \\
\text { richlesto }\end{array}$ \\
\hline \multicolumn{1}{|c|}{ tipo di spazl } & $\begin{array}{c}\text { superficio } \\
\text { utile netta } \mathrm{mq}\end{array}$ & studentin & $\begin{array}{c}\text { mq ' } \\
\text { studente }\end{array}$ & mq/ studente \\
\hline Attivita didattica & & & & \\
\hline Urbanistica & 597,00 & & & \\
\hline Ingegneria & 150,21 & & & \\
\hline Chimica & 130,00 & & & \\
\hline Economia & 234,00 & & & \\
\hline Medicina & 4377,00 & & & \\
\hline Totale superfici attività didattics & 5338,00 & 1530 & 3,5 & 2,6 \\
\hline connettivo e servizi & 2984,11 & 1530 & 2,0 & 1,9 \\
\hline TOTALE & 8322,11 & 1530 & 5,4 & 4,5 \\
\hline
\end{tabular}

Tabella 6 - Progetto. Offerta di spazi complessiva (per corso di laurea).

Nell'ambito del laboratorio si sono poi svolte alcune verifiche di fattibilità del progetto, relative alla stima sommaria dei costi (valutati complessivamente in 17 milioni di euro) e all'analisi finanziaria, che, sia pure in via approssimativa, conferma la convenienza economico-finanziaria dell'investimento. 
$\mathrm{Al}$ di là delle soluzioni prescelte, che possono assumere diverse configurazioni, anche in relazione a più approfondite analisi del complesso, il lavoro comunque dimostra:

- la possibilità di razionalizzare il processo di espansione del Polo e di ricondurre ad un sistema centrato sull'ex ospedale le scelte prese di recente;

- la capacità dell'ospedale di accogliere il Polo universitario in un insediamento di qualità;

- l'effetto positivo dell'insediamento universitario nel tessuto urbano (con la ricucitura di percorsi e l'apertura di spazi pubblici funzionali allo scambio università/città);

- la possibilità di valorizzare un importante monumento trascurato della struttura urbana;

- i costi relativamente contenuti e la convenienza economico-finanziaria dell'intervento. 


\section{L'OSPEDALE DI SAN GIUSEPPE A EMPOLI}
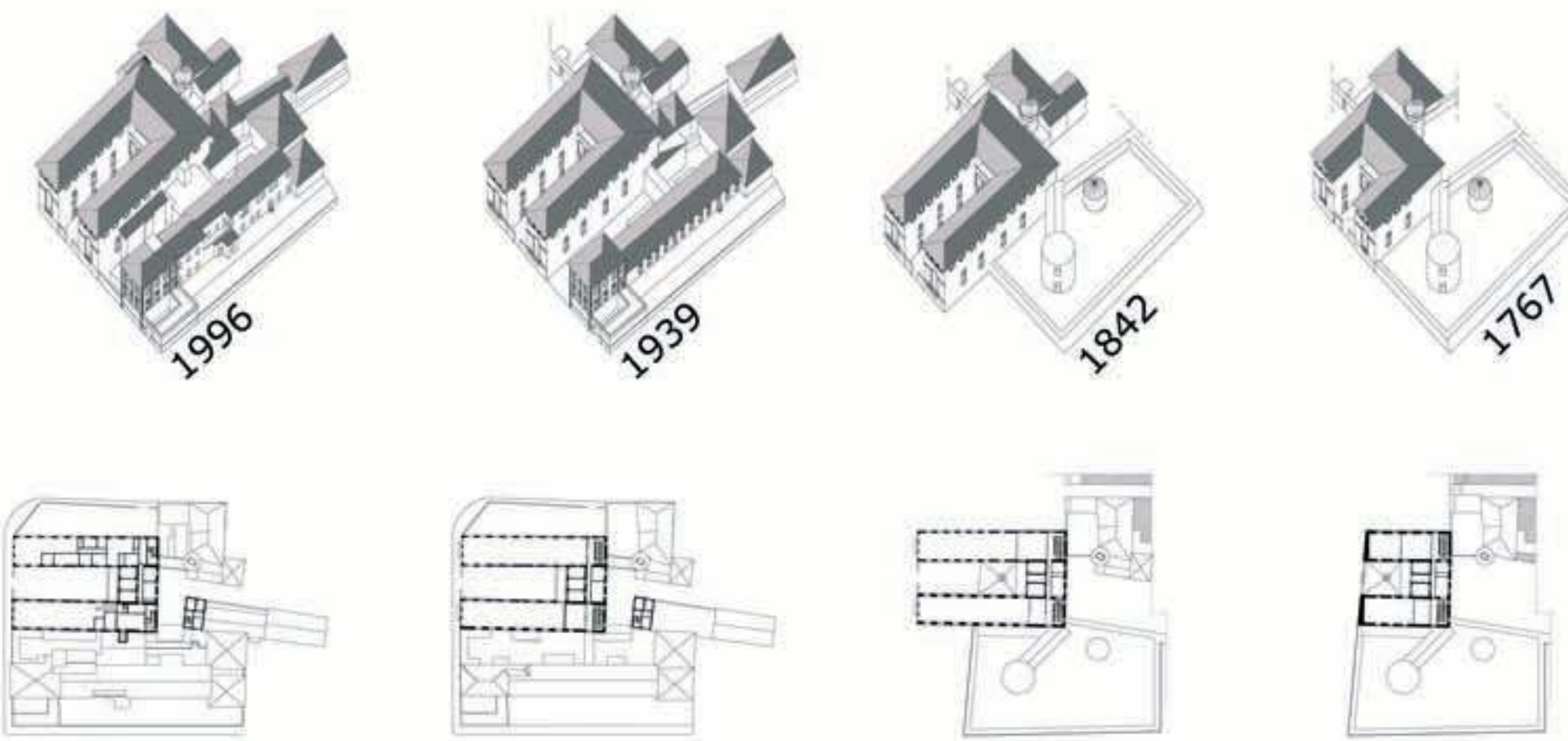

Piano Terzo
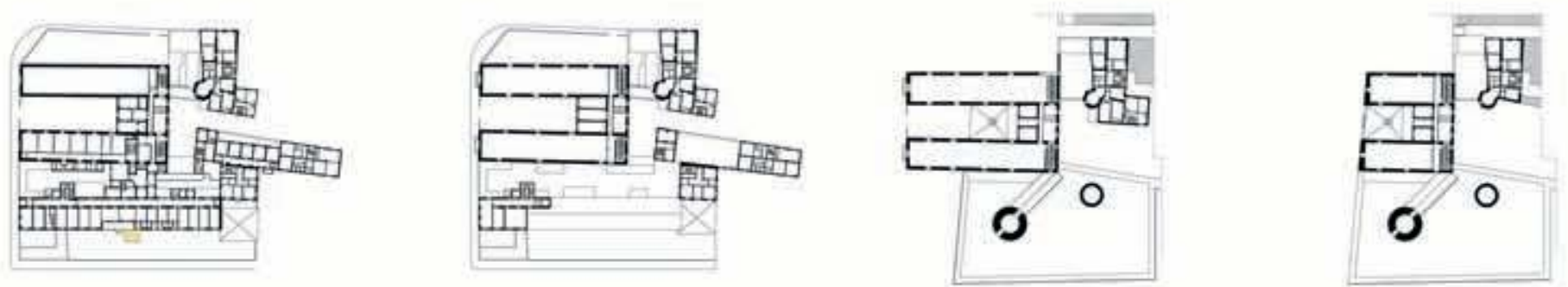

Piano Secondo
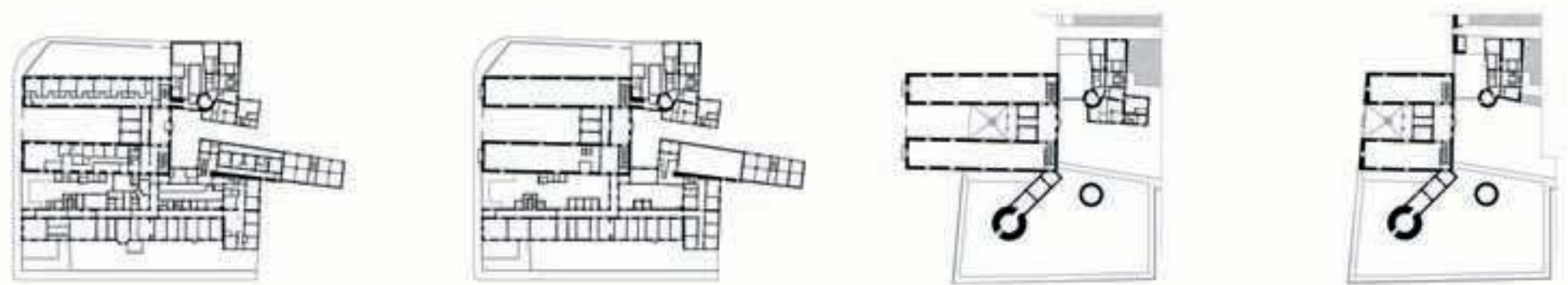

Piano Primo
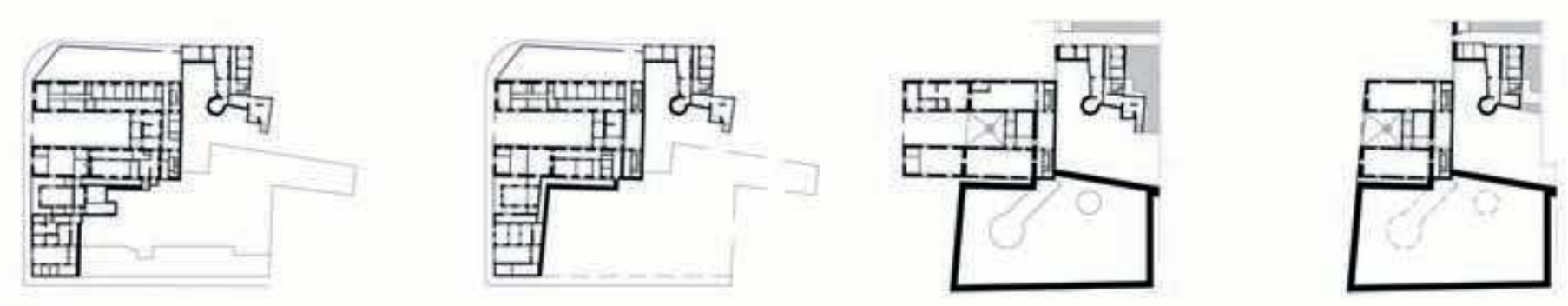